Prepared in cooperation with the Federal Emergency Management Agency

\title{
Total Water Level Data From the January and March 2018 Nor easters for Coastal Areas of New England
}

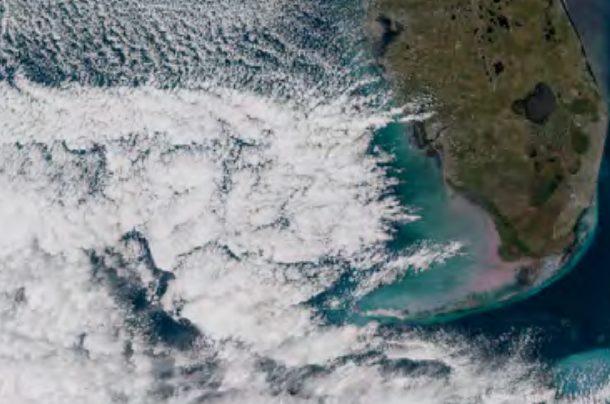

Scientificinvestigations Report $2020=5048$

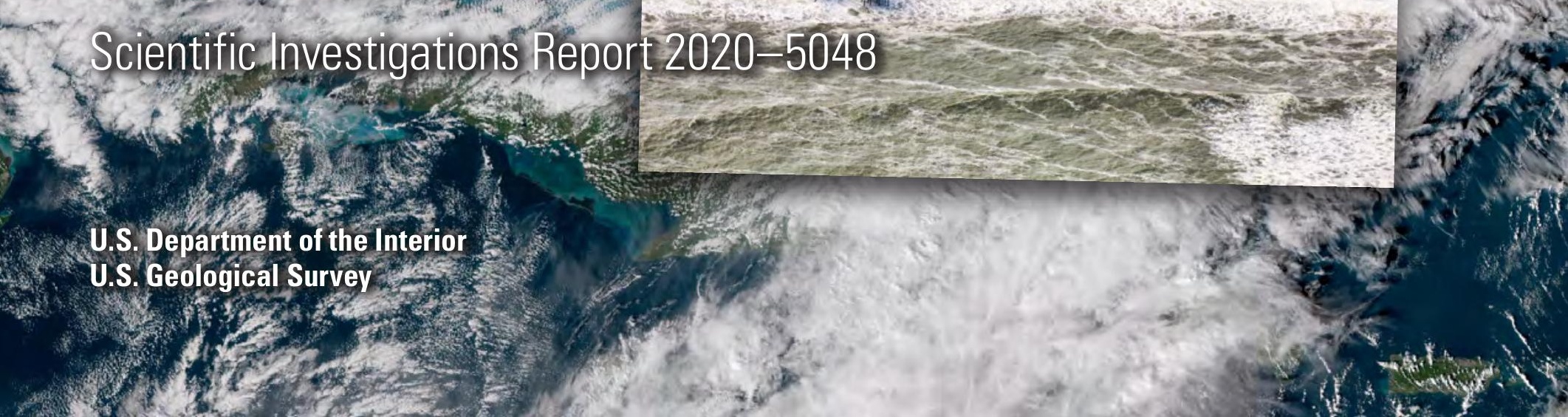


Cover. Background image: Satellite image of nor'easter over the eastern seaboard of the United States on January 4, 2018; image courtesy of National Oceanic and Atmospheric Administration and the Cooperative Institute for Research in the Atmosphere.

Inset images from top to bottom: $A$, Waves crashing into a house in Brant Rock, Marshfield, Massachusetts, on March 6, 2018, near high tide; photograph by the U.S. Geological Survey (USGS). $B$, Waves washing over Peggotty Beach, Scituate, Mass., on March 4, 2018, near high tide; photograph by Karl Swenson, Norwell, Mass.

Back. Ice in Dead Creek salt marsh in Salisbury, Mass., on January 11, 2018; photograph by USGS. 


\section{Total Water Level Data From the January and March 2018 Nor'easters for Coastal Areas of New England}

By Gardner C. Bent and Nicholas J. Taylor

Prepared in cooperation with the Federal Emergency Management Agency

Scientific Investigations Report 2020-5048 


\title{
U.S. Department of the Interior \\ DAVID BERNHARDT, Secretary
}

\author{
U.S. Geological Survey \\ James F. Reilly II, Director
}

U.S. Geological Survey, Reston, Virginia: 2020

For more information on the USGS - the Federal source for science about the Earth, its natural and living resources, natural hazards, and the environment—visit https://www.usgs.gov or call 1-888-ASK-USGS.

For an overview of USGS information products, including maps, imagery, and publications, visit https://store.usgs.gov/.

Any use of trade, firm, or product names is for descriptive purposes only and does not imply endorsement by the U.S. Government.

Although this information product, for the most part, is in the public domain, it also may contain copyrighted materials as noted in the text. Permission to reproduce copyrighted items must be secured from the copyright owner.

Suggested citation:

Bent, G.C., and Taylor, N.J., 2020, Total water level data from the January and March 2018 nor'easters for coastal areas of New England: U.S. Geological Survey Scientific Investigations Report 2020-5048, 47 p., https://doi.org/ 10.3133/sir20205048.

ISSN 2328-0328 (online) 


\section{Acknowledgments}

The authors wish to thank the many private home owners and businesses who allowed access to their property during January, March, September through November 2018 and January 2019, to deploy and retrieve temporary water-level sensors and identify, flag, and survey high-water marks in coastal areas of New England for the January and March 2018 nor'easters. The authors also thank the following U.S. Geological Survey field personnel who deployed and retrieved temporary water-level and barometric pressure sensors and identified, flagged, and surveyed high-water marks: Joseph Hatfield, Brittney Izbicki, Dee-Anne McCarthy, Patrick McNamara, Rachel Pearson, Nigel Pepin, Colin Sweeney, and David Thompson in Connecticut and Rhode Island; Mike Beardsley and Henry Gilman in Maine; Salvador Amador, David Armstrong, Samuel Banas, Alexander Bissell, Robert Bradley, Christopher Bruet, Joshua Combs, Monica Conlin, James Fisher, Rena Kalmon, Peiran Liang, Andrew Massey, Anthony Motta, Gordon McQuaid, Brian Nelson, Stephen O'Brien, Lance Ramsbey, Cory Smith, Blake Stone, Dale Tom, Nathan Van Meter, Alexander Wong, and Curtis Yaeger in Massachusetts; and Glenn Berwick, Chandlee Keirstead, Richard Kiah, Domenic Murino, Paul Shea, and Sanborn Ward in New Hampshire. 



\section{Contents}

Acknowledgments ……...................................................................................................................

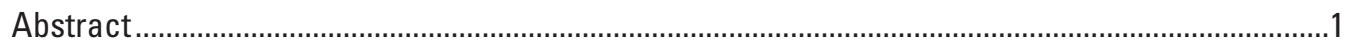

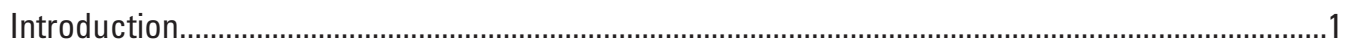

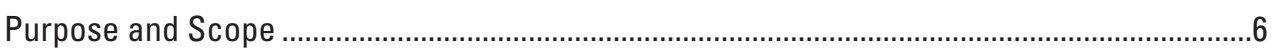

Study Area

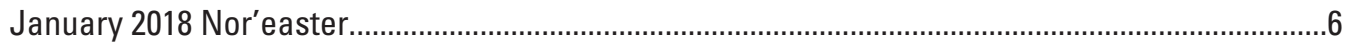

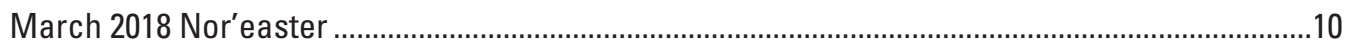

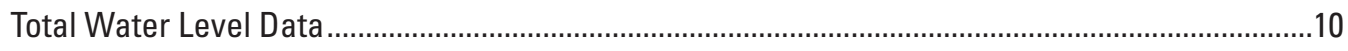

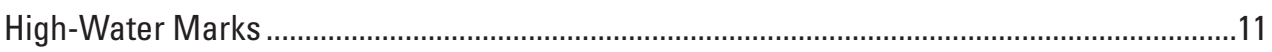

Identification and Flagging ............................................................................................11

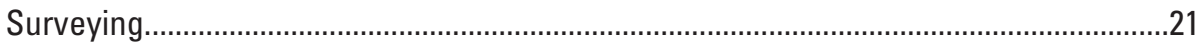

January 2018 Total Water Level Elevations .............................................................................22

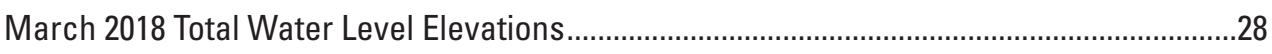

Comparison of January and March 2018 Total Water Level Elevations ....................................28

Comparison of Observed and Predicted Total Water Level Elevations ...................................32

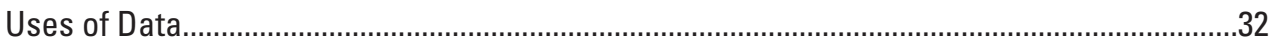

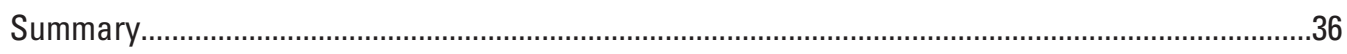

References Cited...................................................................................................................37

Appendix 1. Quality Assurance of Survey Equipment Used To Determine Elevations of High-Water Marks From the January and March 2018 Nor'easters ...................................41

\section{Figures}

1. Map showing locations of gages and temporary water-level sensors for total water level data in New England during the January and March 2018 nor'easters.........3

2. Graphs showing observed and predicted total water level elevations at the Boston and Portland tide gages from January 1 to 7 and from March 1 to 7, 2018..........4

3. Map showing locations of surveyed high-water marks from the January 2018 nor'easter in coastal areas of eastern Massachusetts ....................................................7

4. Maps showing locations of surveyed high-water marks from the March 2018 nor'easter in coastal areas of New England....................................................................

5. Photographs showing evidence and field methods for recording high-water marks caused by the January and March 2018 nor'easters in coastal areas of New England .

6. Boxplots showing total water level elevations for the January and March 2018 nor'easters from Portland, Maine to Cape Cod Bay, Massachusetts .....

\section{Tables}

1. Description of tide gages and coastal streamgages affected tidally or by backwater from tides with total water level data during the January and March 2018 nor'easters in New England.

2. Description of U.S. Geological Survey temporary water-level sensors deployed during the March 2-4, 2018, nor'easter in New England . 
3. Uncertainty of high-water marks for coastal areas of New England

4. High-water marks with no elevation or elevation determined to be inaccurate from the January and March 2018 nor'easters in New England

5. Summary of total water level elevations for high-water marks in coastal areas of Massachusetts following January 2018 nor'easter of and coastal areas of Massachusetts, Connecticut, Rhode Island, New Hampshire, and Maine following the March 2018 nor'easter.

6. Summary of total water level elevations at tide gages and coastal streamgages affected by storm tides or tidal backwater during the January and March 2018 nor'easters in New England..

7. Summary of total water level elevations at U.S. Geological Survey temporary storm-tide sensors during the March 2-4, 2018, nor'easter in New England ..

8. Approximate storm surge at 26 tide gages during the January and March 2018 nor'easters in New England

\section{Conversion Factors}

U.S. customary units to International System of Units

\begin{tabular}{lll}
\hline \multicolumn{1}{c}{ Multiply } & By & \multicolumn{1}{c}{ To obtain } \\
\hline inch (in.) & 2.54 & centimeter $(\mathrm{cm})$ \\
foot (ft) & 0.305 & meter $(\mathrm{m})$ \\
mile (mi) & 1.609 & kilometer $(\mathrm{km})$ \\
square mile $\left(\mathrm{mi}^{2}\right)$ & 2.59 & square kilometer $\left(\mathrm{km}^{2}\right)$ \\
mile per hour $(\mathrm{mi} / \mathrm{h})$ & 1.609 & kilometer per hour $(\mathrm{km} / \mathrm{h})$ \\
\hline
\end{tabular}

\section{Datum}

Vertical coordinate information is referenced to the North American Vertical Datum of 1988 (NAVD 88) and the National Geodetic Vertical Datum of 1929 (NGVD 29).

Horizontal coordinate information is referenced to the North American Datum of 1983 (NAD 83).

Elevation, as used in this report, refers to distance above the vertical datum. 


\section{Abbreviations}

$\begin{array}{ll}\text { FEMA } & \text { Federal Emergency Management Agency } \\ \text { GNSS } & \text { Global Navigation Satellite System } \\ \text { GPS } & \text { Global Positioning System } \\ \text { HWM } & \text { high-water mark } \\ \text { MassDOT } & \text { Massachusetts Department of Transportation } \\ \text { NAD 83 } & \text { North American Datum of 1983 } \\ \text { NGVD 29 } & \text { National Geodetic Vertical Datum of 1929 } \\ \text { NAVD 88 } & \text { North American Vertical Datum of 1988 } \\ \text { NGS } & \text { National Geodetic Survey } \\ \text { NOAA } & \text { National Oceanic and Atmospheric Administration } \\ \text { RTKN } & \text { real-time kinematic network } \\ \text { STN } & \text { short-term network } \\ \text { USACE } & \text { U.S. Army Corps of Engineers } \\ \text { USGS } & \text { U.S. Geological Survey }\end{array}$





\title{
Total Water Level Data From the January and March 2018 Nor'easters for Coastal Areas of New England
}

\author{
By Gardner C. Bent and Nicholas J. Taylor
}

\section{Abstract}

During winter 2017-18 coastal areas of New England were impacted by the January 4, and March 2-4, 2018, nor' easters. The U.S. Geological Survey (USGS), under an interagency agreement with the Federal Emergency Management Agency (FEMA), collected total water level data (the combination of tide, storm surge, wave runup and setup, and freshwater input) using the North American Vertical Datum of 1988 (NAVD 88) from high-water marks and continuous water-level sensors, to better understand the areal extent, timing, and impact of coastal flooding from strong storms.

During the January 4, 2018, nor'easter the National Oceanic and Atmospheric Administration (NOAA) Boston, Massachusetts, tide gage recorded the highest total water level on record of $9.66 \mathrm{ft}$. During the March 2-4, 2018, nor'easter, the Boston tide gage recorded its third highest total water level on record of $9.16 \mathrm{ft}$.

After the January and March 2018 nor'easter storms, the USGS deployed field teams that identified and flagged highwater marks along the coastlines of eastern Massachusetts in January and from Portland, Maine, south to the ConnecticutNew York State border in March. In preparation for the approach of the March 2018 nor'easter, the USGS deployed 35 temporary water-level sensors along the coastline of New England to collect total water level data during the storm. Total water level data were also collected at 28 tide gages and 14 coastal streamgages (affected tidally or by tidal backwater during coastal storms) in New England during both nor'easters.

Total water level elevations at 71 high-water marks collected after the January 2018 nor'easter in coastal areas of eastern Massachusetts ranged from 5.8 to 15.1 feet (ft), with an average elevation of $9.4 \mathrm{ft}$ and a median elevation of $9.6 \mathrm{ft}$. Total water level elevations at 10 tide gages and 7 coastal streamgages from Portland to Cape Cod Bay ranged from 4.8 to $11.2 \mathrm{ft}$, with an average of $9.1 \mathrm{ft}$ and a median of $9.6 \mathrm{ft}$. Following the March 2018 nor'easter, 111 high-water marks were collected along the New England coastline. Of the 111 high-water marks, 100 were along the eastern coastline of New England from Portland to Cape Cod and had elevations that ranged from 5.3 to $15.1 \mathrm{ft}$, with an average of $8.9 \mathrm{ft}$ and a median of $8.6 \mathrm{ft}$. The remaining 11 high-water marks along the southern coastline of New England in Connecticut, Rhode Island, and Massachusetts had elevations that ranged from 3.1 to $7.5 \mathrm{ft}$, with an average of $4.3 \mathrm{ft}$ and a median of $4.9 \mathrm{ft}$. Total water level elevations for 19 USGS temporary water-level sensors from Portland to Cape Cod Bay ranged from 6.2 to $10.4 \mathrm{ft}$, with an average of $8.4 \mathrm{ft}$ and a median of $8.7 \mathrm{ft}$. Total water level elevations at 10 tide gages and 6 coastal streamgages from Portland to Cape Cod Bay ranged from 7.8 to $10.8 \mathrm{ft}$, with an average of $9.1 \mathrm{ft}$ and a median of $9.2 \mathrm{ft}$.

There were 10 tide gages and 5 coastal streamgages with data from both nor'easters from Portland to Cape Cod Bay; for the January nor'easter, the average and median elevations were about 0.3 and $0.5 \mathrm{ft}$ higher, respectively, than for the March nor'easter. At the 52 high-water mark locations with data for both nor'easters in Massachusetts, the average and median elevations were 0.1 and $0.4 \mathrm{ft}$ higher, respectively, for the January nor'easter than for the March nor'easter.

At 10 tide gages along the coastline from Portland to Cape Cod Bay, the observed peak total water level elevations for the January nor'easter ranged from 1.6 to $3.7 \mathrm{ft}$ higher than the concurrent predicted elevations, with an average of $2.8 \mathrm{ft}$ and a median of $3.0 \mathrm{ft}$ higher. For the March nor'easter, the observed peak total water level elevations ranged from 1.8 to $4.0 \mathrm{ft}$ higher than the concurrent predicted elevations, with an average of $2.7 \mathrm{ft}$ and a median of $3.0 \mathrm{ft}$ higher. This is approximately the amount of storm surge that was experienced during the highest tides of the two nor'easters along the coastline from Portland to Cape Cod Bay.

\section{Introduction}

On January 4, 2018, a strong nor'easter passed off the coast of New England. This nor'easter developed and tracked up the Atlantic Ocean coast quickly, having developed from a low-pressure system off the coast of Florida and the Bahamas on January 3. The rapid intensification of this storm and the loss of 59 millibars (mb) of pressure in 24 hours to a low of $950 \mathrm{mb}$ produced peak wind gusts from 50 to 70 miles per hour (mi/h). This storm also occurred during a period 
of astronomical high tides from a full moon on January 1, 2018. The National Oceanic and Atmospheric Administration (NOAA) Boston, Massachusetts, tide gage (station 8443970; fig. 1, station no. 16) recorded a peak total water level ${ }^{1}$ elevation of 9.66 feet ( $\mathrm{ft}$ ) above the North American Vertical Datum of 1988 (NAVD 88) on January 4 (fig. 2A; National Oceanic and Atmospheric Administration, 2019b), which was the highest elevation in the 97-year record (since May 1921; National Oceanic and Atmospheric Administration, 2018a). The previous peak of record had been $9.52 \mathrm{ft}$ above NAVD 88 , which was observed during the February 7, 1978, nor'easter. Additionally, the NOAA Portland, Maine, tide gage (station 8418150; fig. 1, station no. 8) recorded its highest total water level elevation of $8.42 \mathrm{ft}$ above NAVD 88 on January 4 (fig. 2B; National Oceanic and Atmospheric Administration, 2019c). This is the third highest elevation on record since 1912 (National Oceanic and Atmospheric Administration, 2018a).

Flooding and wind damage affected communities in Massachusetts on the North Shore area between Boston and New Hampshire, at Boston Harbor, on the South Coast area between Boston and Cape Cod, along Cape Cod Bay, and on Nantucket, Mass. (New England Cable News, 2018). By January 30, the Massachusetts Emergency Management Agency reported preliminary estimates of damages of about $\$ 9.5$ million (Charles Baker, Governor, Commonwealth of Massachusetts, written commun., 2018). A major disaster declaration was not made, although a request for a 60-day extension in the application was submitted from Massachusetts Governor Charles Baker to the Federal Emergency Management Agency (FEMA) headquarters (Michelle O'Toole, Massachusetts Emergency Management, oral commun., 2018).

On March 2-4, another strong nor' easter passed off the coast of New England. This nor'easter started as a stationary front over the Midwest on March 1 and moved eastward off the mid-Atlantic coast, resulting in a new low-pressure system that formed rapidly in the Atlantic and then moved up along the coast of New England. This storm underwent a rapid intensification, losing $26 \mathrm{mb}$ of pressure in 21 hours and reaching a low of $947 \mathrm{mb}$. This storm also occurred during a period of astronomical high tides from a full moon on March 1. The NOAA Boston tide gage (8443970; fig. 1, station no. 16) recorded its highest total water level elevation of $9.16 \mathrm{ft}$ above NAVD 88 on March 2, 2018 (fig. 2C; National Oceanic and Atmospheric Administration, 2019b), which is the third highest elevation on record (National Oceanic and Atmospheric Administration, 2018a). The NOAA Portland tide gage (8418150; fig. 1, station no. 8) recorded its highest total water level elevation of $7.91 \mathrm{ft}$ above NAVD 88 on March 2 (fig. 2D; National Oceanic and Atmospheric Administration, 2019c), which is the seventh highest elevation on record (National Oceanic and Atmospheric Administration, 2018a).

\footnotetext{
1The term "total water level" data is used because some data maybe a combination of astronomical tides, storm surge, waves (runup and setup), and freshwater inputs (Federal Emergency Management Agency, 2016; National Oceanic and Atmospheric Administration, 2019a).
}

For the March nor'easter, reported costs in Massachusetts were more than $\$ 23.8$ million for emergency response, debris clearing, and repairs for public infrastructure; more than 2,000 homes were damaged and almost 150 homes were destroyed (Wade, 2018). For the March 2-4, 2018, nor'easter major disaster declarations were made for areas of Massachusetts, New Hampshire, and Maine. The declaration for Massachusetts (DR-4372) was for a "severe winter storm and flooding on March 2-3, 2018" in Essex, Norfolk, Plymouth, Barnstable, Nantucket, and Bristol Counties (Federal Emergency Management Agency, 2018b). More than $\$ 3.4$ million of total public assistance grants was obligated. A major disaster declaration was issued for Maine (DR-4367) for a "severe storm and flooding on March 2-8, 2018" in York County (Federal Emergency Management Agency, 2018a). More than \$2.7 million of total public assistance grants were obligated. A major disaster declaration was issued for New Hampshire (DR-4370) for a "severe storm and flooding on March 2-8, 2018" in Rockingham County (Federal Emergency Management Agency, 2018c). More than $\$ 400,000$ of total public assistance grants were obligated.

The eastern coastline of New England has been affected by many nor'easters. Before the January and March 2018 nor'easters, some of the most damaging nor'easters since the February 1978 (Gadoury, 1979) nor'easter have been the October 1991 (Kedzierski, 1992), December 1992, and January 2015 (Massey and Verdi, 2015) nor'easters. All 6 of these nor'easters have total water level elevations that rank in the highest 10 peak total water level elevations at the NOAA tide gage in Boston (National Oceanic and Atmospheric Administration, 2018a). In 2012, Hurricane Sandy damaged the coastline of eastern Massachusetts (part of the damage to the southern coast of New England; Ostiguy and others, 2018).

In response to the January 2018 nor'easter, the U.S. Geological Survey (USGS) identified, flagged, and surveyed 85 high-water marks (HWMs) along the eastern coastline of Massachusetts from the State border with New Hampshire to Cape Cod Bay. For the March 2018 nor'easter, the USGS deployed temporary water-level and barometric pressure sensors and identified, flagged, and surveyed 115 HWMs along the coastline of New England. Continuous total water level elevations were also recorded for both the January and March 2018 nor'easters along the New England coastline by 28 tide gages operated each variously by the USGS, NOAA, or U.S. Army Corps of Engineers (USACE) and at 14 coastal streamgages affected tidally or by tidal backwater during coastal storms (hereafter referred to as "coastal streamgages"), operated by the USGS. 


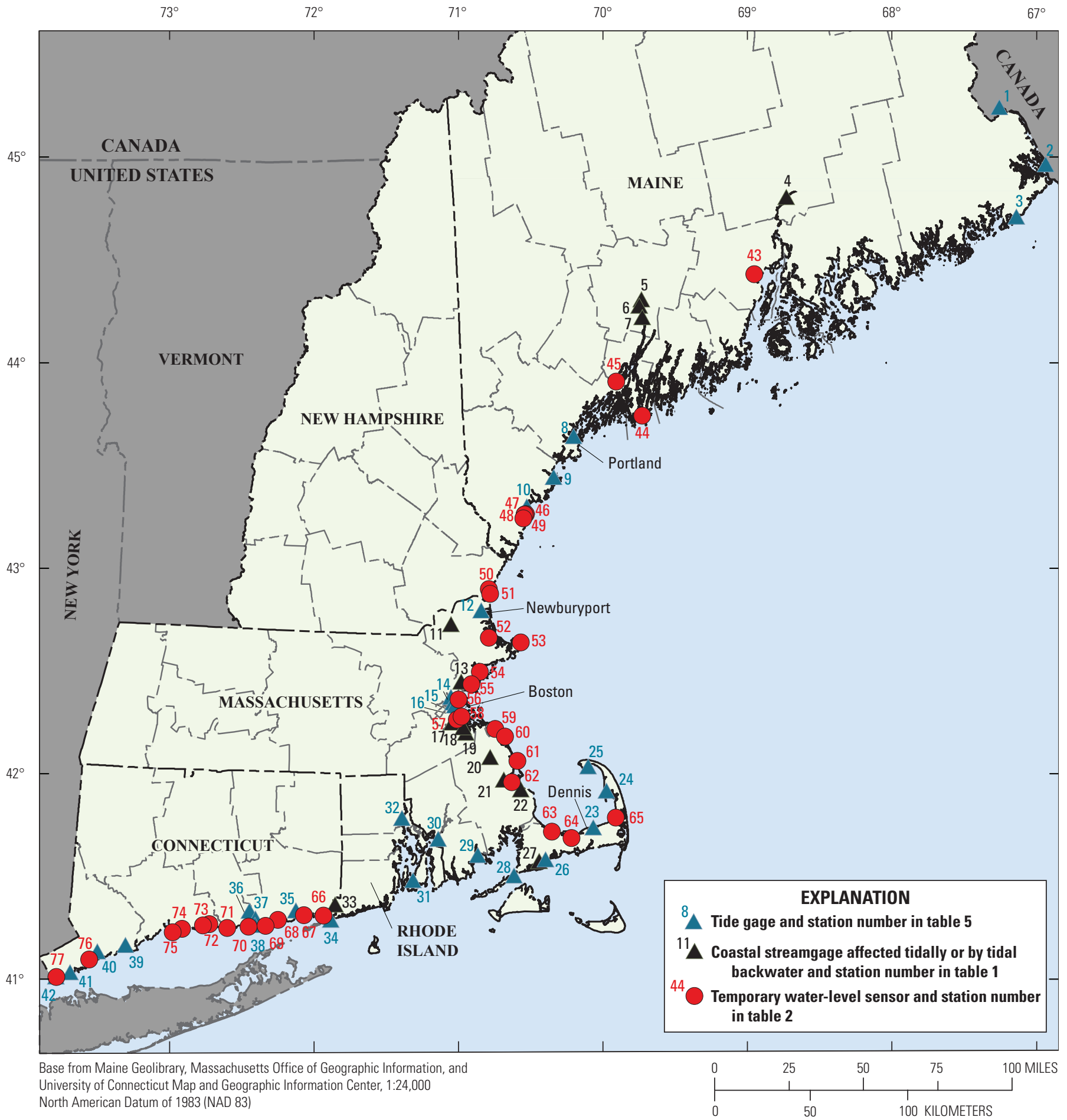

Figure 1. Locations of 28 tide gages, 14 coastal streamgages affected tidally or by tidal backwater, and temporary water-level sensors where total water level data were collected in New England during the January and March 2018 nor'easters. 
A. Boston, Mass.

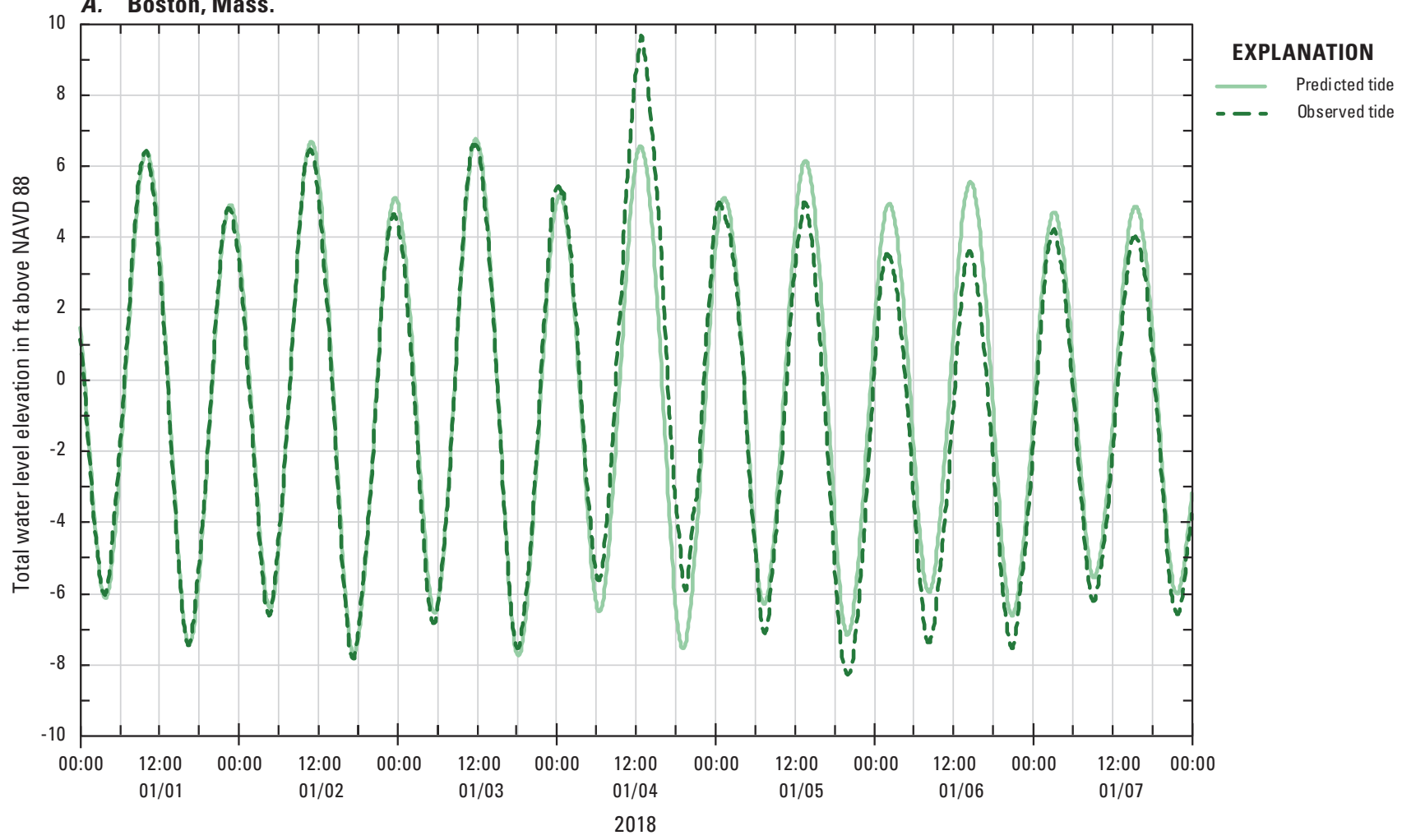

B. Portland, Maine

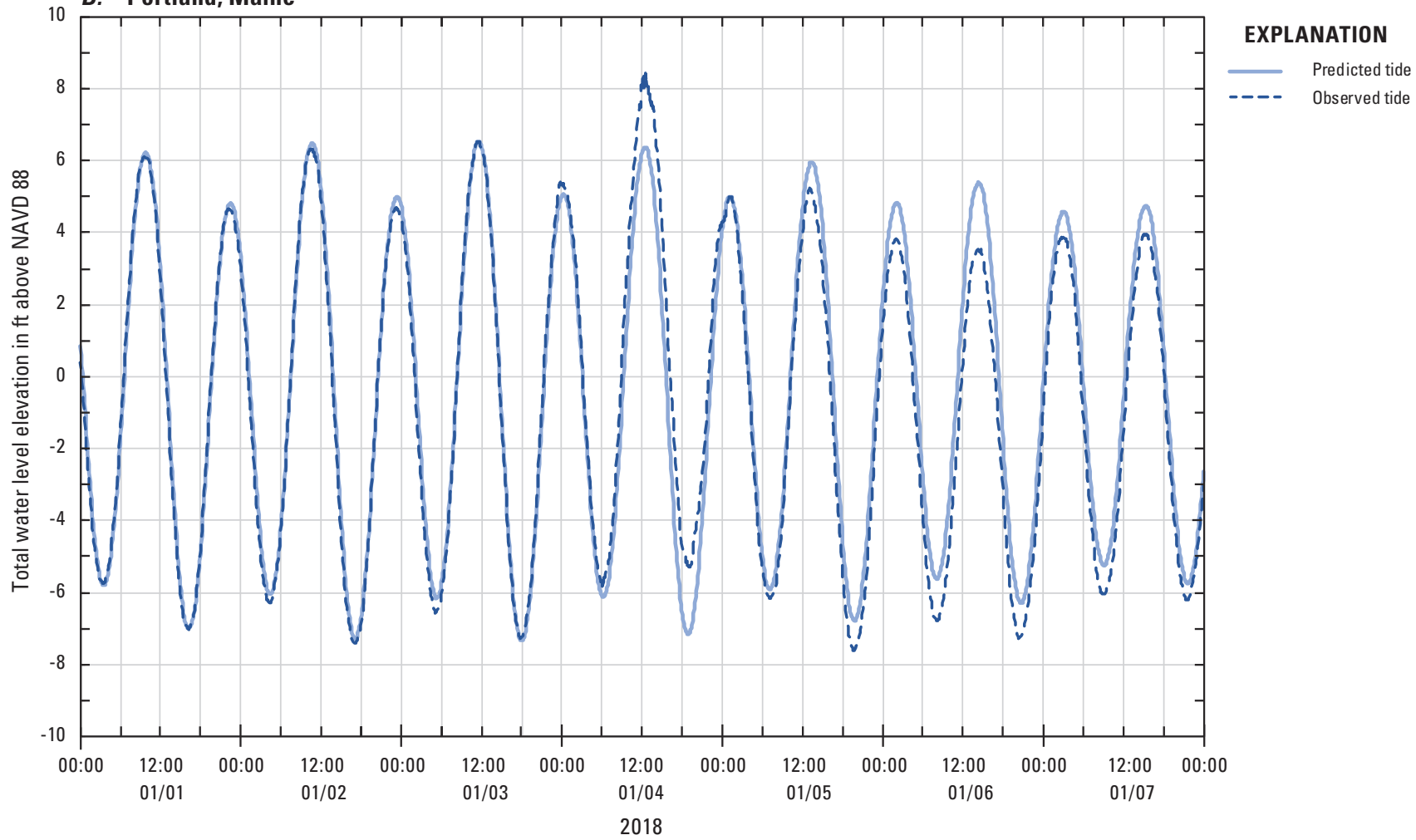

Figure 2. Observed and predicted total water level elevations at the National Oceanic and Atmospheric Administration Boston, Massachusetts, and Portland, Maine, tide gages $A$ and $B$, from January 1 to 7 and $C$ and $D$, from March 1 to 7, 2018. Tide gages are described in table 1 (station numbers 16 and 8, respectively), and locations are shown on figure 1. ft, foot; NAVD 88, North American Vertical Datum of 1988. 
C. Boston, Mass

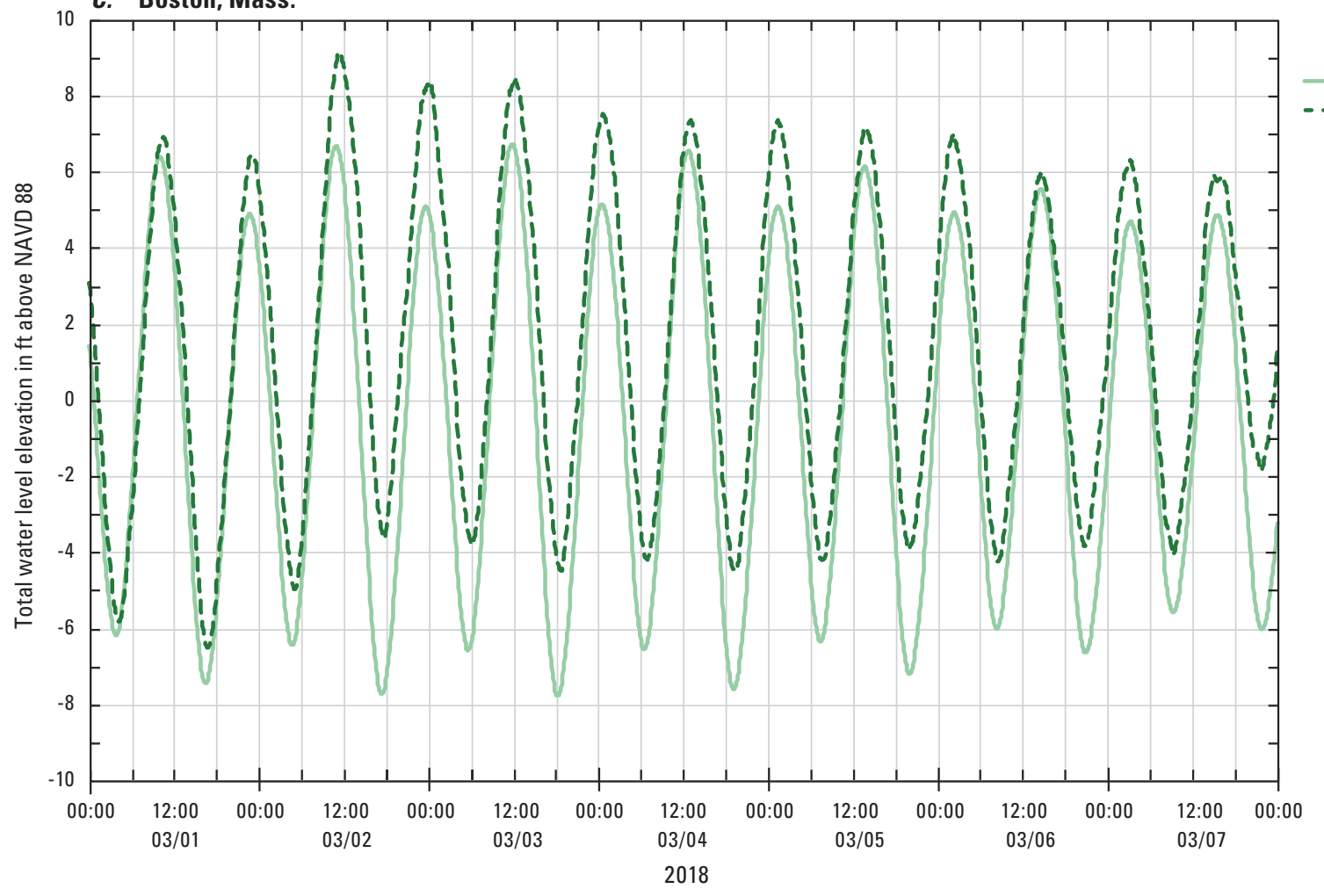

EXPLANATION

Predicted tide

Observed tide

D. Portland, Maine

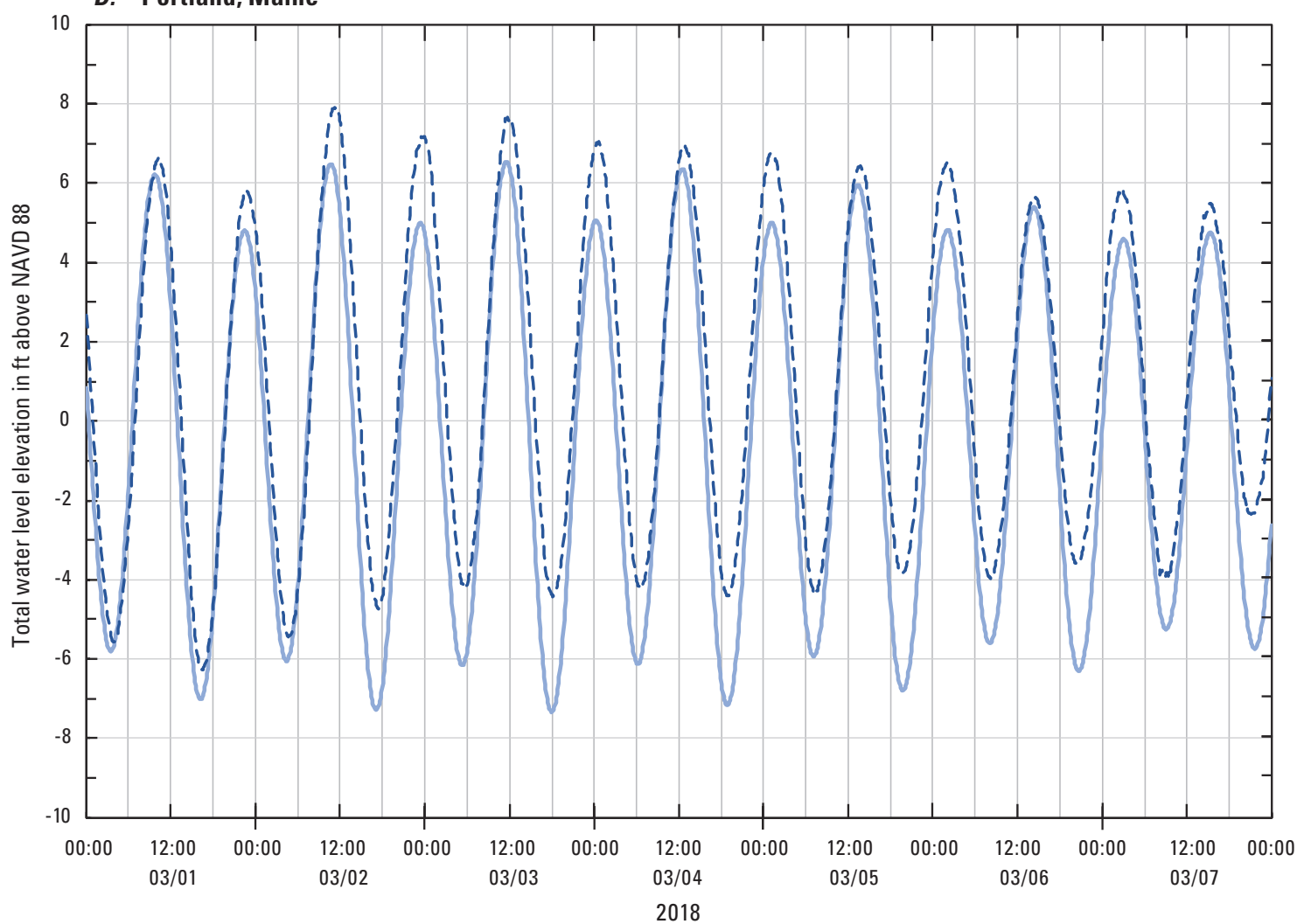

EXPLANATION

Predicted tide

- - - - Observed tide

Figure 2. - Continued 
Total Water Level Data from 2018 Nor'easters for Coastal Areas of New England

\section{Purpose and Scope}

This report documents and provides total water level data for HWMs, tidal gages, coastal streamgages, and temporary water-level sensors for the January 4 and the March 2-4, 2018, nor'easters in the coastal areas of New England. In response to two nor'easters, the USGS through an interagency agreement with FEMA flagged and surveyed HWMs along the hardest hit coastal areas in New England to enhance understanding of the areal extent and impact of coastal flooding resulting from strong storms. USGS water-level sensors also were deployed prior to the March 2018 nor'easter to record total water level elevations before, during, and following the storm. Total water level elevation data for HWMs were collected at or near the temporary water-level sensors and tide gages as well as at other selected locations, including many at locations documented for the February 1978 nor'easter. HWMs were surveyed for selected locations immediately following the two nor'easters and then at the remaining sites from September to November 2018 and in February 2019.

Total water level elevations for all HWMs, tide gages, coastal streamgages, and temporary water-level sensors for the nor'easters of January and March 2018 are presented, summarized, and compared. Observed high total water level elevations are also compared with corresponding NOAApredicted high elevations. The emphasis in this study is on the total water level data collected along the eastern coastline of New England between Portland and Cape Cod Bay. Individual information on all USGS total water level data are available on the USGS Flood Event Viewer website for the January 2018 nor'easter (https://stn.wim.usgs.gov/FEV/ \#NoreasterJanuary2018) and the March 2018 nor'easter (https://stn.wim.usgs.gov/FEV/\#NoreasterofMarch2018).

\section{Study Area}

Coastal flooding from nor'easters primarily affects the eastern coast of New England (Massachusetts, New Hampshire, and Maine) because the winds are mainly from the northeast. Many of the top 10 peak total water level elevations on record at the long-term tide gages in Boston and Portland (National Oceanic and Atmospheric Administration, 2018a) are from nor'easters. However, in New England, nor'easters can also push ocean waters into coastal inlets that are open to the east, such as Long Island Sound between Connecticut and New York, or into coastal areas in Connecticut and Rhode Island that are open to the ocean and face a more easterly direction. Total water level data were mainly collected from Portland to Cape Cod Bay during both the January and March 2018 nor'easters; some data were also collected along the remaining New England coastline. The New England coastline is quite long, with the tidal shorelines for the five New England States being 618 miles (mi) for Connecticut,
$384 \mathrm{mi}$ for Rhode Island, 1,519 mi for Massachusetts, $131 \mathrm{mi}$ New Hampshire, and 3,478 mi for Maine (National Oceanic and Atmospheric Administration, 2019d).

For the January 2018 nor'easter, HWMs were collected in the coastal areas of eastern Massachusetts from the State border with New Hampshire to Cape Cod Bay (fig. 3). During the January nor'easter, total water level data were also collected at 28 tide gages and 14 coastal streamgages (fig. 1).

For the March 2018 nor'easter, HWMs were collected in coastal areas of Connecticut, Rhode Island, Massachusetts, New Hampshire, and Maine (fig. 4). During this nor'easter, USGS temporary water-level sensors were deployed to collect continuous total water level data 1 to 2 days before, during, and for a few days following the nor'easter. Additionally, total water level data were collected at 28 tide gages and 14 coastal streamgages (fig. 1).

\section{January 2018 Nor'easter}

On January 3, the National Weather Service (NWS) observed the rapid genesis of a low-pressure system about $200 \mathrm{mi}$ off the eastern coast of Florida, which was predicted to bring severe winter weather to the eastern seaboard of the United States (National Weather Service, 2018a, summary 1; 2018c). The storm was a strong nor'easter by the morning of January 4 when it reached New England. Nor'easters have counterclockwise winds and so bring strong winds from a northeasterly direction, which often batter coastal areas along the eastern seaboard. This storm was accompanied by a rapid drop in barometric pressure, nearly $59 \mathrm{mb}$ in 24 hours (Erdman, 2018) - to a low of $950 \mathrm{mb}$ (National Weather Service, 2018a, summary 5). As a result, coastal areas of New England were impacted by high winds with peak wind gusts from about 50 to $75 \mathrm{mi} / \mathrm{h}$ (National Weather Service, 2018a, summary 8). Nantucket had a recorded peak wind gust of $76 \mathrm{mi} / \mathrm{h}$. Snowfall amounts ranged from about 10 to 20 inches across New England, with central Maine receiving up to 22 inches (National Weather Service, 2018a, summary 8). The strong northeastern winds combined with a period of high astronomical tides from the full moon on January 1 caused storm surge and coastal flooding.

Extensive flooding stretched along the eastern coast, concentrated along the eastern coastline of New England (Samenow, 2018). Damage estimates of about $\$ 9.5$ million were received by the Massachusetts Emergency Management Agency by the end of January 2018 (Governor Charles Baker, Commonwealth of Massachusetts, written commun., 2018). Many coastal communities from Portland to Cape Cod Bay were flooded by ocean water that surged inland over beaches and sea walls and up coastal tidal rivers (New England Cable News, 2018). 


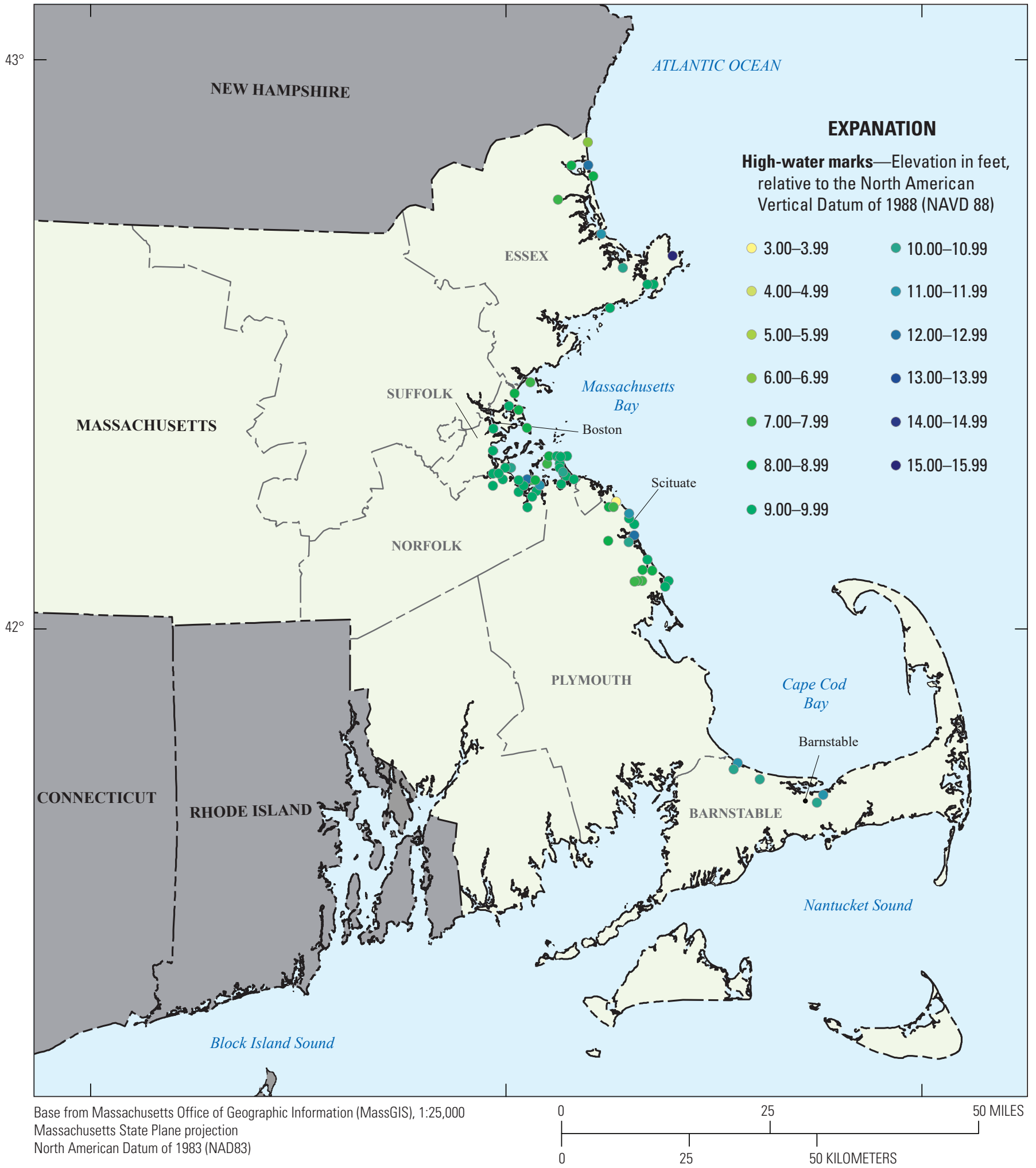

Figure 3. Locations of surveyed high-water marks from the January 2018 nor'easter in coastal areas of eastern Massachusetts. 


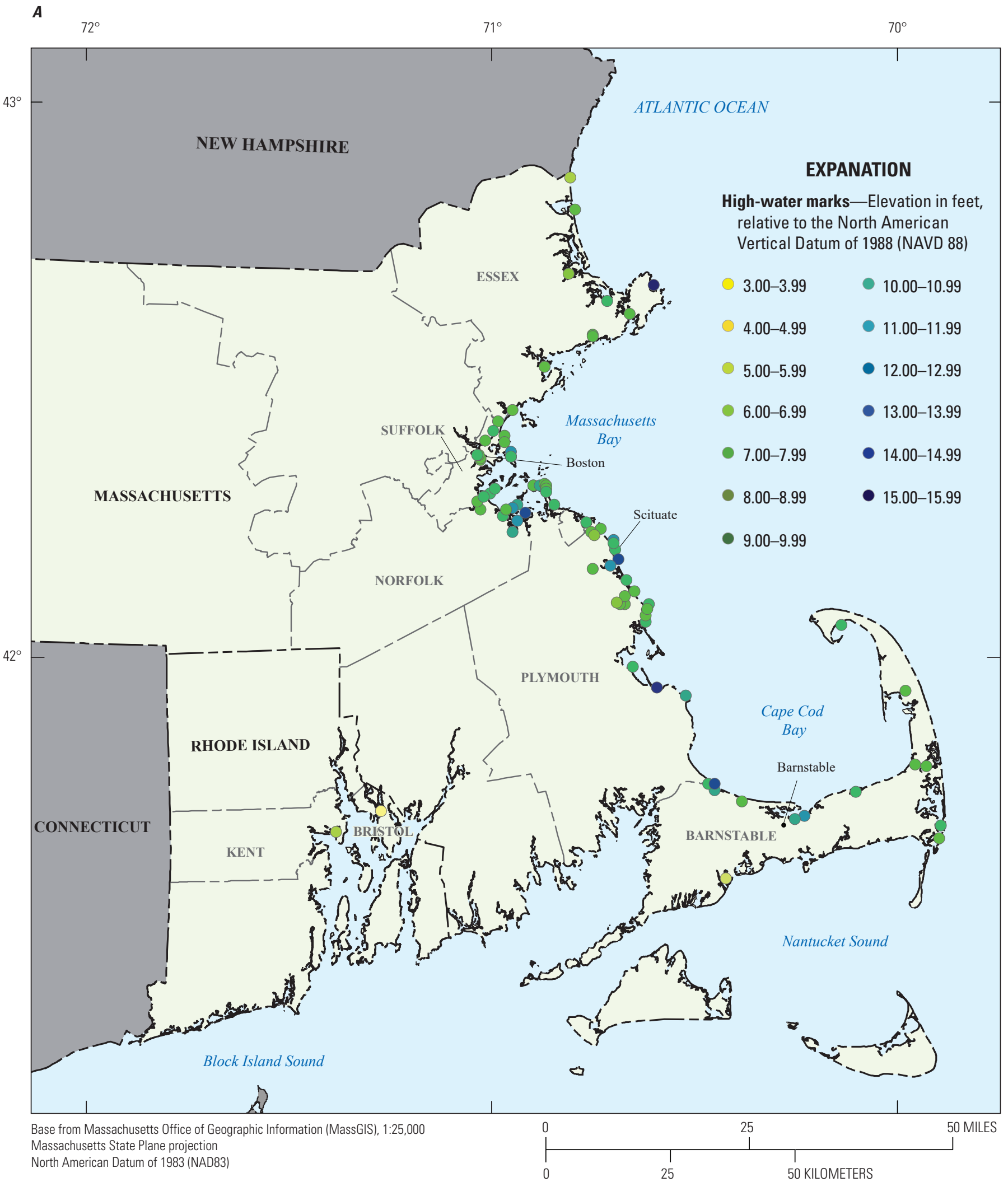

Figure 4. Locations of surveyed high-water marks from the March 2018 nor'easter in coastal areas of $A$, Massachusetts and Rhode Island, $B$, New Hampshire and Maine, and $C$, Connecticut. 


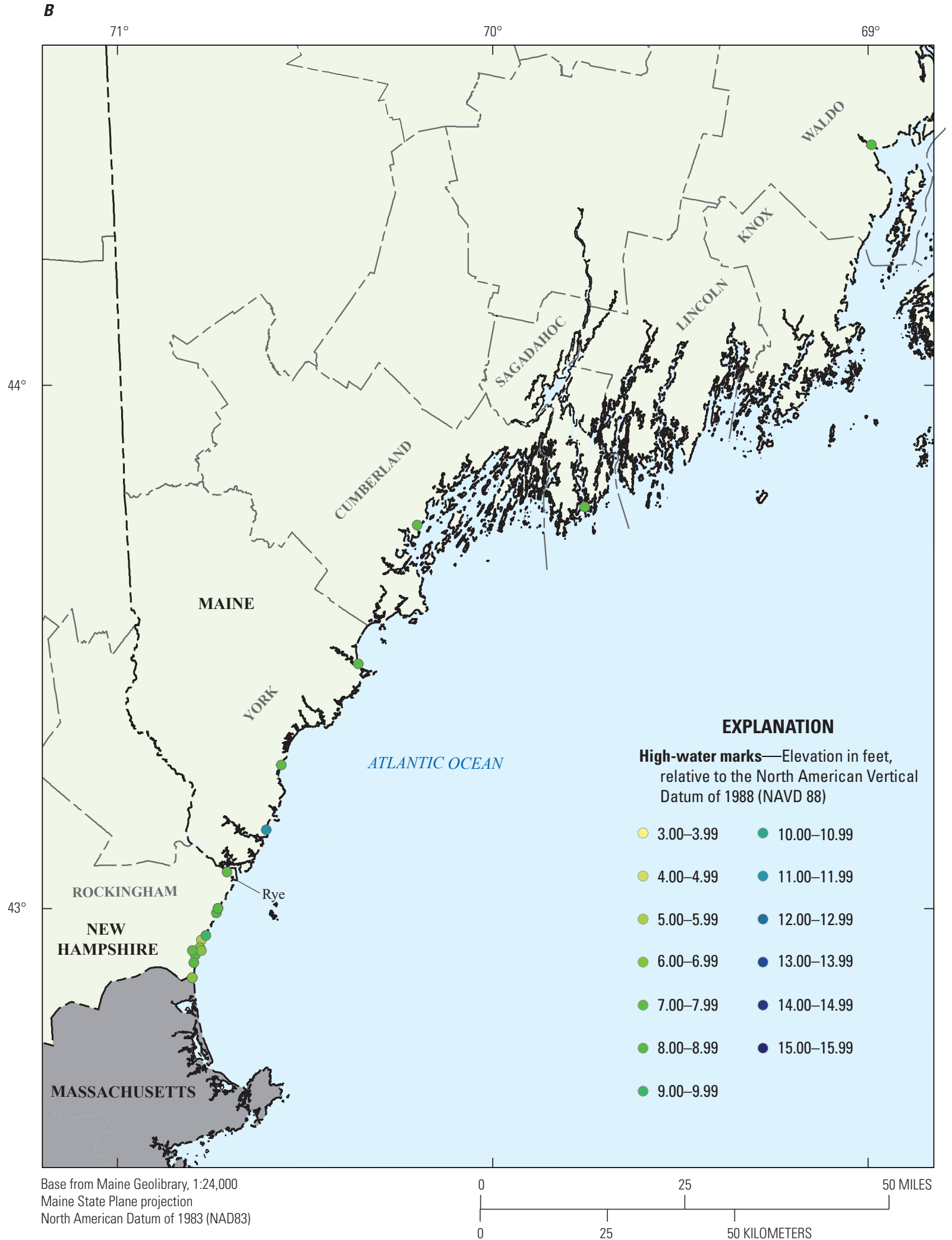

Figure 4. - Continued 


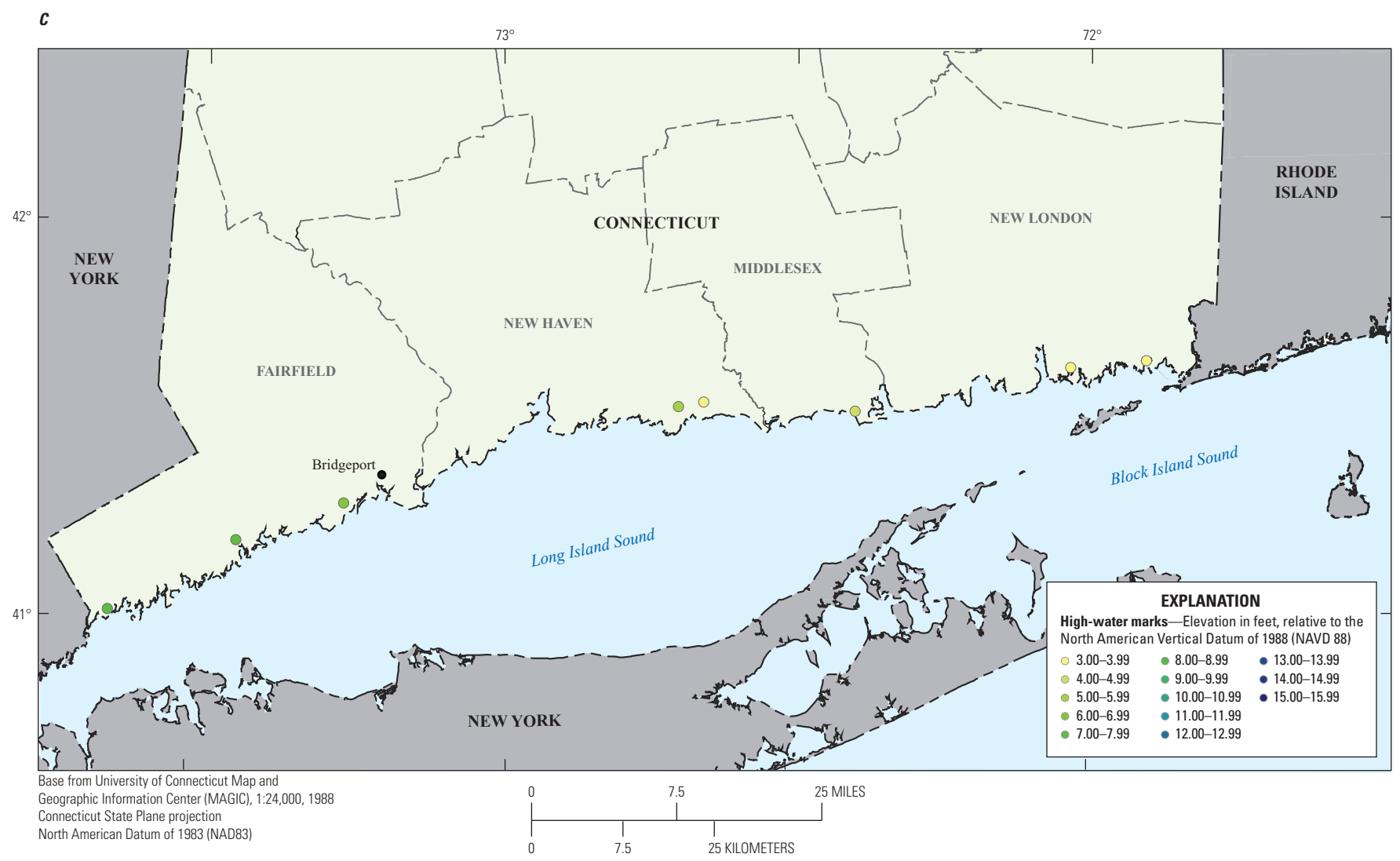

Figure 4. - Continued

\section{March 2018 Nor'easter}

In early March, a second nor'easter of a similar magnitude as the January nor'easter affected coastal communities along the eastern seaboard from the mid-Atlantic to New England. On the evening of March 1, the NWS observed a low-pressure system in Pennsylvania migrating east and rapidly strengthening off the mid-Atlantic coastline (National Weather Service, 2018b, summary 1). By March 2, a rapid drop in barometric pressure of nearly $26 \mathrm{mb}$ in 24 hours was recorded for the storm, to a low of $974 \mathrm{mb}$ (Weather Channel, The, 2018). Later that same day, the NWS issued winter-storm and high-wind warnings from Pennsylvania to New England and a blizzard warning for upstate New York to northeastern Pennsylvania (National Weather Service, 2018b, summary 4). Peak wind gusts ranged from about 40 to $90 \mathrm{mi} / \mathrm{h}$ from Georgia to New England (National Weather Service, 2018b, summary 7). Inland areas of the northeast from Pennsylvania to New England had snowfall totals ranging from a few inches up to 39 inches in upstate New York, and the coastal areas had rainfall amounts ranging from 1 to almost 6 inches. The persistent strong winds during the highest astronomical tide of the month, due to a full moon on March 1, caused widespread flooding in coastal areas of New England, especially on the eastern-facing coastline (Di Liberto, 2018). Even as the storm continued moving offshore northeastward, long-period swells exacerbated coastal flooding over multiple tidal cycles from March 3 to 5. Eight deaths were reported from downed trees across the northeast and mid-Atlantic (Caron, 2018). Major disaster declarations were made, and public assistance grants were obligated for $\$ 3.4$ million, $\$ 400,000$, and $\$ 2.7$ million for areas of Massachusetts, New Hampshire, and Maine, respectively (Federal Emergency Management Agency, 2018a, b, c).

\section{Total Water Level Data}

Total water level data were collected for the January and March 2018 nor'easters from tide gages, coastal streamgages, temporary water-level sensors, and HWMs. Data from tide gages operated by NOAA are typically referred to as "stormtide" data, which is the astronomical tide plus storm surge. The USGS and USACE tide gages could possibly include other effects from waves and are thus typically referred to as total water levels. The filtered elevation data at USGS temporary water-level sensor (often referred to as "storm-tide sensor") are typically storm-tide data. HWMs were collected in varying coastal environments and thus may contain effects 
from waves (wave runup and setup) and possibly freshwater inputs for HWMs further upstream on a tidal river, in addition to the storm surge.

The USGS, NOAA, and USACE currently [2020] operate a network of about 28 continuous tide gages in New England (table 1). Augmenting this tidal network, the USGS also currently [2020] operates about 14 continuous coastal streamgages. Additionally, for coastal storms (nor'easters and hurricanes) for which moderate to severe coastal flooding is expected, the USGS deploys temporary water-level sensors at selected locations to record detailed continuous total water level elevations before, during, and following the storm (Verdi and others, 2017). During the March 2018 nor'easter, the USGS deployed 35 temporary water-level sensors along the New England coastline (table 2). Additionally, following a coastal storm that results in severe coastal flooding, the USGS (typically in cooperation with FEMA) identifies, flags, and surveys HWMs to determine the highest total water level elevations at coastal locations not covered by sensors. The USGS also collects HWMs near tide gages and temporary water-level sensors to confirm the recorded peak total water level elevations. The coastal streamgages are equipped with a crest-stage gage (Sauer and Turnipseed, 2010; U.S. Geological Survey, 2019a) to verify the recorded peak total water level elevation.

\section{High-Water Marks}

Evidence of the highest water surface elevation (fig. 5) during a riverine or coastal flood is known as a HWM (Benson and Dalrymple, 1967). The highest water level is often documented by deposits of natural and manmade pieces of debris (seeds, grasses, tree needles or leaves, woody material, sediment, trash, or ice) that are transported by floodwaters or waves, and deposited on the sides of buildings, fences, mailboxes, telephone poles, and trees (Koenig and others, 2016; Feaster and Koenig, 2017). These types of deposits are common during flooding and are used as evidence in identifying the highest water level (fig. $5 C-F$ ). After the peak of a flood, the water recedes and leaves material that forms a HWM through settling, staining, or adhesion. Well-defined HWMs form best in areas of slow water movement. It is necessary to obtain the location of HWMs as soon as the peak water levels begin to recede. Time is critical to finding the best available HWMs at each site before the marks are faded, lost, moved, removed, or covered up by natural (rain, wind, and sun) and manmade alterations.

Additionally, if very cold temperatures persist during and following a flood, the highest water level may also be documented by ice or snow lines on the sides of buildings, fences, mailboxes, telephone poles, or trees (fig. $5 A$ and $B$ ). These ice or snow lines can also include natural and manmade pieces of debris frozen into them, which aid in identification of the highest water level. Timely observations are also important following the peak water levels recess because these marks can quickly melt or be altered by temperatures above or near freezing.

Using procedures described by Benson and Dalrymple (1967), Koenig and others (2016), and Feaster and Koenig (2017), HWMs were identified, flagged, and documented for the peak total water level elevations from the January and March 2018 nor'easters. Information on the approximate horizontal location, the type and general accuracy of the HWM, and approximate elevation above the land surface were documented so they could be found by surveyors later. Additionally, a sketch map was drawn, and photographs were taken (fig. 5).

\section{Identification and Flagging}

In preparation of surveying and flagging HWMs following the peak total water level elevations of the January 2018 nor'easter, potential HWM locations were selected in communities in and around Boston that were identified as having been affected by coastal flooding (Kerry Bogdan, FEMA, written commun., 2018). Potential HWMs were also selected in several other communities north and south of Boston and along Cape Cod Bay that were identified as having been affected by coastal flooding (Sarah White, Massachusetts Emergency Management Agency, written commun., 2018). When possible, HWM sites were selected to be near locations where HWMs were flagged and surveyed following the February 1978 nor'easter (Gadoury, 1979) and October 1991 nor'easter (Kedzierski, 1992); near a USGS temporary waterlevel sensor site (Verdi and others, 2017); or in an area with known damages from previous coastal flooding. A similar procedure was followed for the March nor'easter.

For the January nor'easter, two-person crews went into the field from January 6 to 10 to identify and flag HWMs along the coastlines of Massachusetts. For the March 2018 nor'easter, crews identified and flagged HWMs along the coastlines from Connecticut to Maine from March 5 to 9. Where situations prohibited the identification of HWMs with acceptable quality at a planned site, a nearby site of better quality was selected and identified by the field crew.

Once an adequate HWM was found, the field crew used a standard field form to record each mark with a unique identification number, the road and town or city location, a description of the location of the HWM relative to a landmark, the latitude and longitude of the HWM, a description of the HWM (seeds, grasses, tree needles, leaves, woody material, sediment, ice, or melted ice or snow), the distance of the HWM above ground surface or other described object, a qualitative description of the accuracy of the HWM, and the type of marker (USGS HWM disk, paint, permanent marker, PK nail, nail, chisel mark, stake, or surveyor's hub) used to flag the location. Field crews rated and assigned HWM general assessments using a qualitative scale: excellent, good, fair, and poor (table 3). 


\section{A. MAPLY24223}

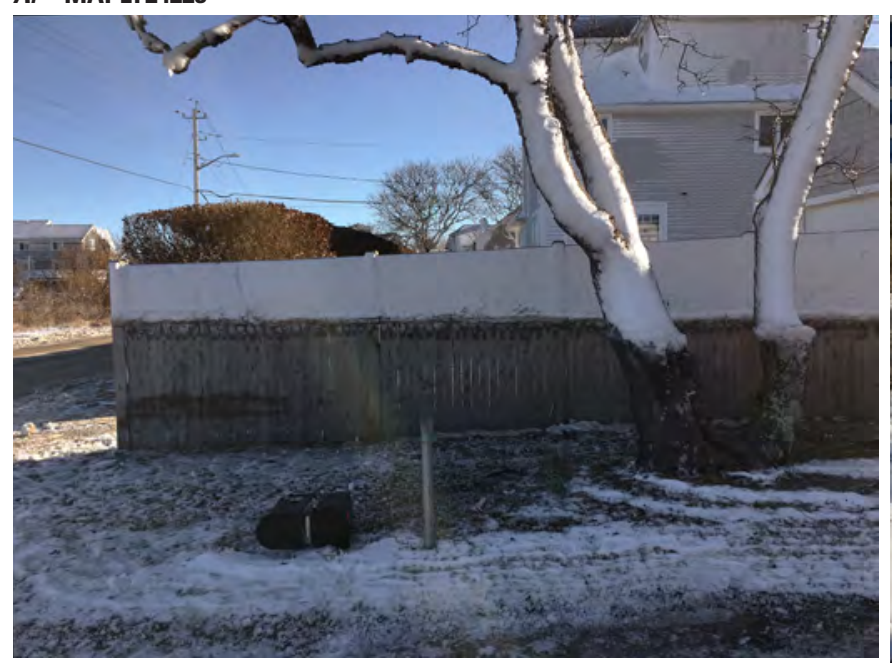

\section{CTFAI04807}

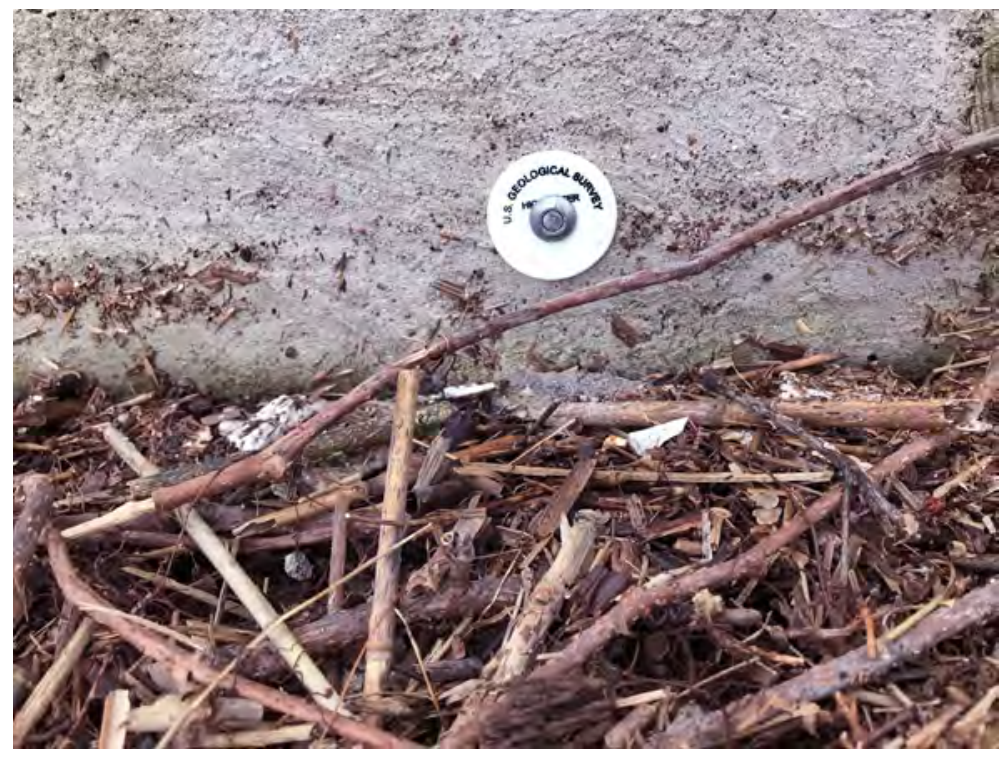

Figure 5. Evidence and field methods for recording high-water marks caused by the January and March 2018 nor'easters in coastal areas of New England. A, Ice line on wooden fence (January 2018, Scituate, Massachusetts); B, ice line on Neponset River pier (January 2018, Boston, Mass.); C, white disc bolted to concrete wall at a debris line (March 2018, Bridgeport, Connecticut); D, green disk at wrack line on road surface (March 2018, Barnstable, Mass.) and green disk with orange paint line at January 2018 high-water mark; E, debris and wrack line on side of building (March 2018, Scituate, Mass.); and F, mud line on wooden fence (March 2018, Rye, New Hampshire). High-water marks depicted can be found on the U.S. Geological Survey Flood Event Viewer (https://stn.wim.usgs.gov/FEV/) by their event date and Short-Term Network number shown in the label of each photograph.

\section{B. MASUF04853}

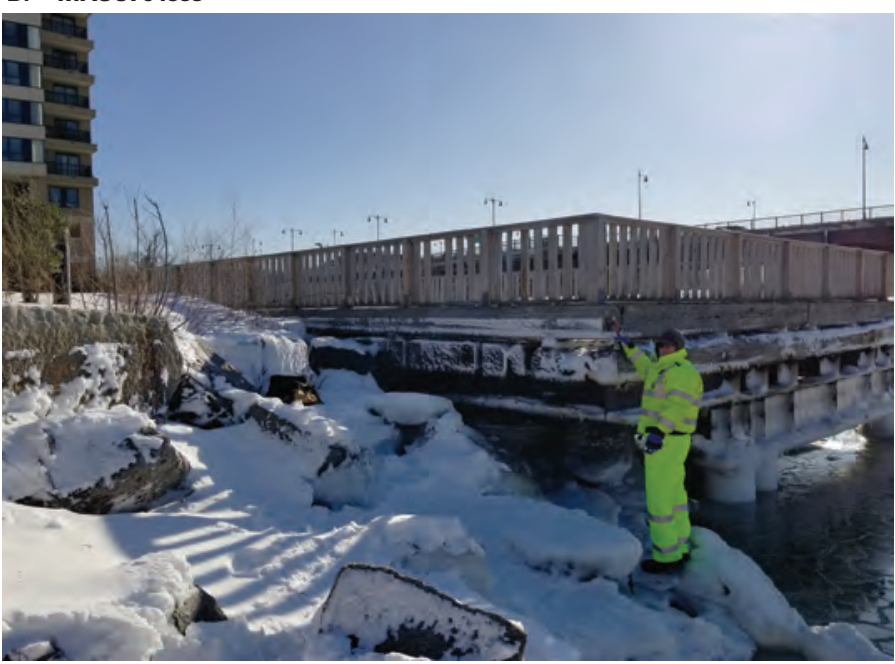

D. MABAR24109

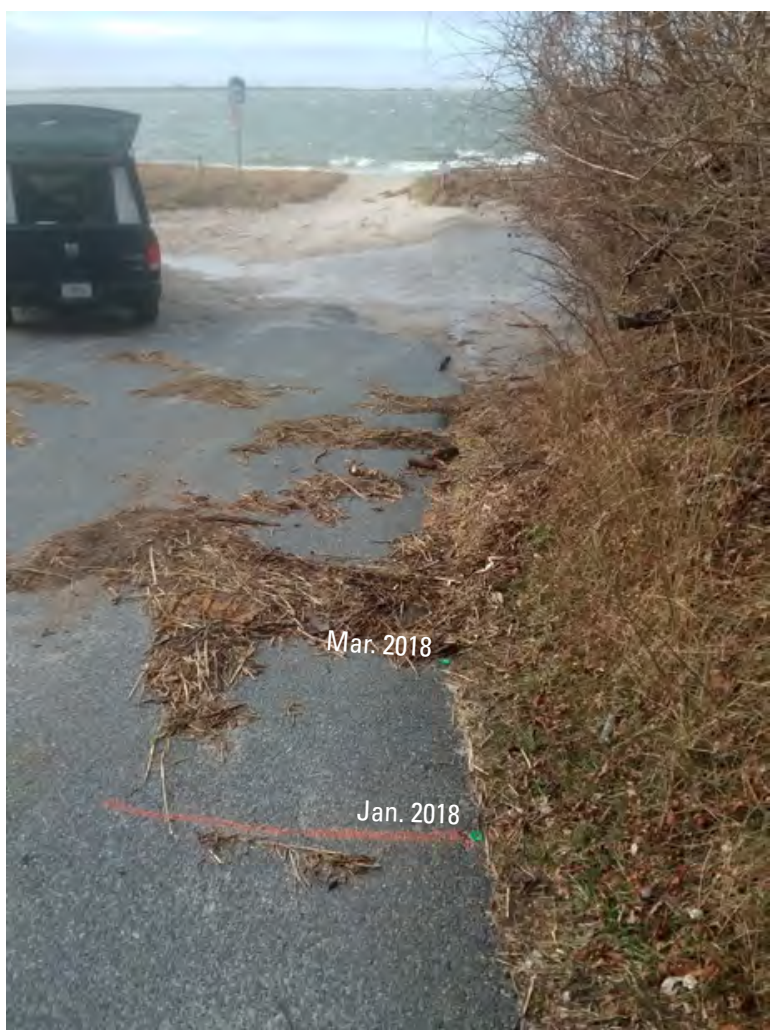




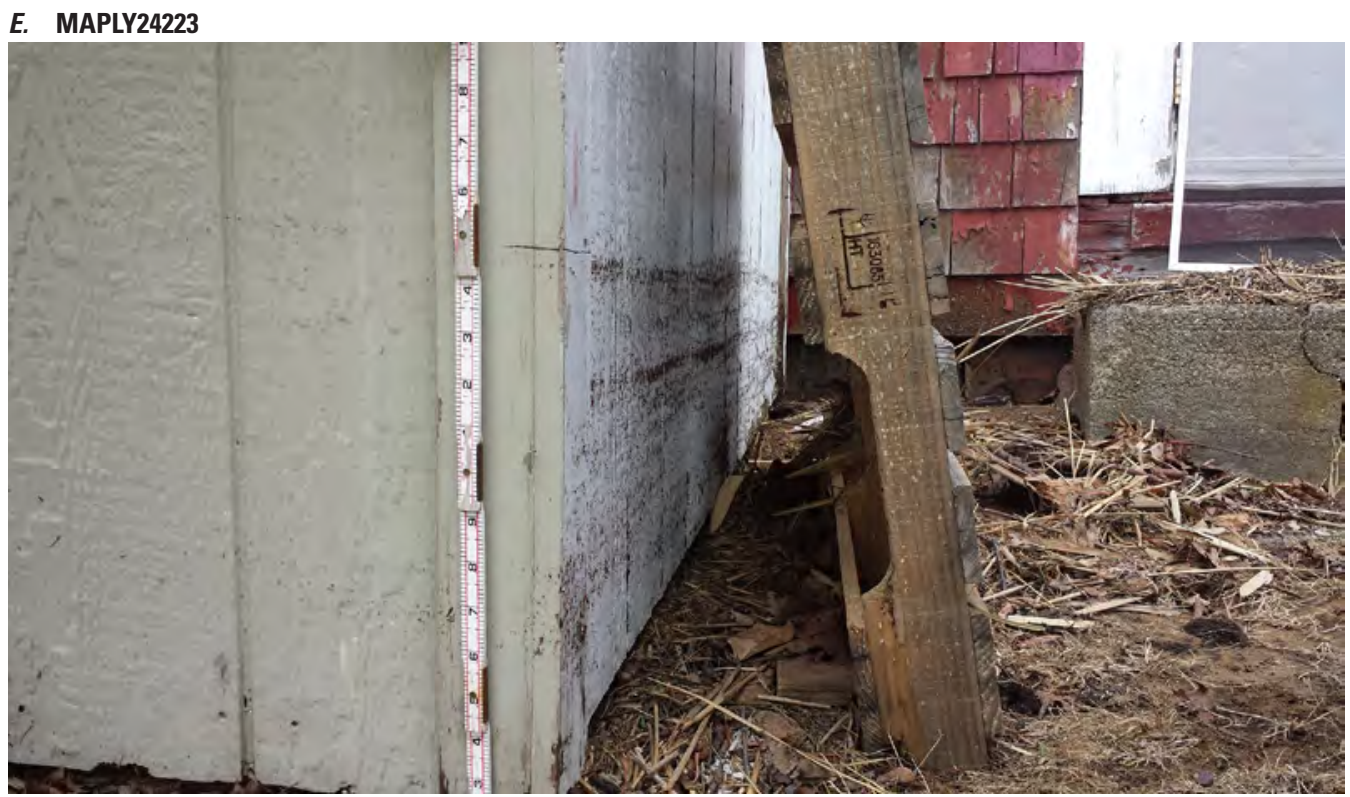

\section{F. NHROC24227}

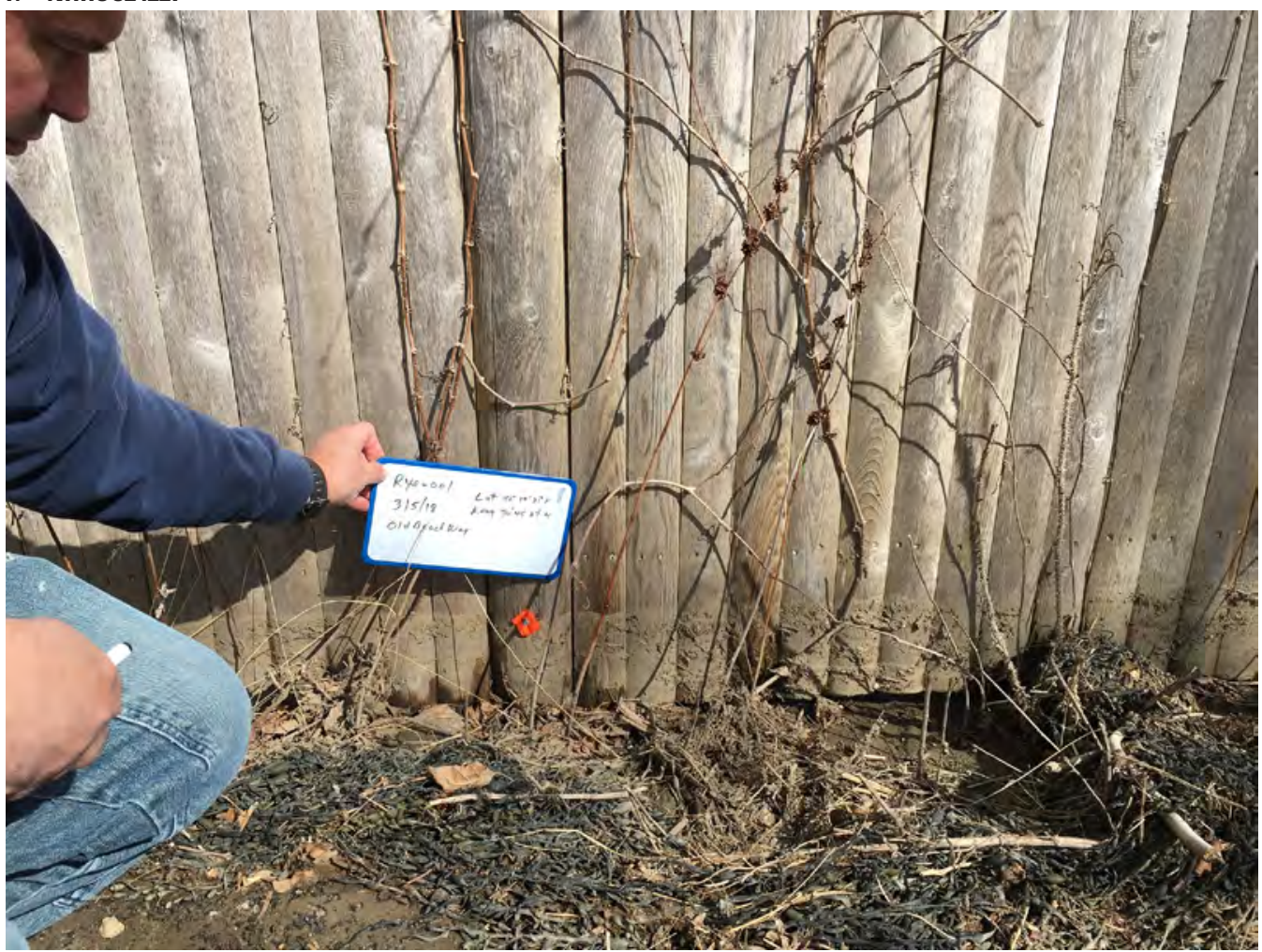

Figure 5. - Continued 
Table 1. Description of tide gages and coastal streamgages affected tidally or by backwater from tides with total water level data during the January and March 2018 nor'easters in New England.

[Station numbers (nos.) and locations of tide gages and coastal streamgages affected tidally or by backwater from tides are shown on figure 1. Tide gages operated by the U.S. Geological Survey (USGS) and the U.S. Army Corps of Engineers (USACE) list the paired National Oceanic and Atmospheric Administration (NOAA) tide gage for which predicted tidal data are available on the line below each of those tide gages. NOAA tide gages use the same gage for predicted tide data. USGS streamgages do not have a paired NOAA tide gage. Data for USGS tide gages and coastal streamgages are from U.S. Geological Survey (2019d); for NOAA tide gages, from National Oceanic and Atmospheric Administration (2019e); and for USACE tide gages, from U.S. Army Corps of Engineers (2019). ft, foot; NAVD 88, North American Vertical Datum of 1988; NGVD 29, National Geodetic Vertical Datum of 1929; MLLW, mean lower-low water; mi, mile; CT, Connecticut; MA, Massachusetts; ME, Maine; NH, New Hampshire; RI, Rhode Island; Rt., Route; St., Street; -, no number]

\begin{tabular}{|c|c|c|c|c|c|c|c|c|c|}
\hline $\begin{array}{l}\text { Stat } \\
\text { ion } \\
\text { no. }\end{array}$ & Gage type & $\begin{array}{l}\text { Agency } \\
\text { operat- } \\
\text { ing gage }\end{array}$ & $\begin{array}{l}\text { USGS or NOAA } \\
\text { station no. }\end{array}$ & Station name & $\begin{array}{l}\text { Latitude, } \\
\text { in decimal } \\
\text { degrees }\end{array}$ & $\begin{array}{l}\text { Longitude, } \\
\text { in decimal } \\
\text { degrees }\end{array}$ & $\begin{array}{l}\text { Period of } \\
\text { record }\end{array}$ & Remarks & $\begin{array}{c}\text { Conver } \\
\text { sion from } \\
\text { gage } \\
\text { datum to } \\
\text { NAVD 88, } \\
\text { in ft }\end{array}$ \\
\hline \multicolumn{10}{|c|}{ Maine, sites north of Portland } \\
\hline \multirow[t]{2}{*}{1} & \multirow[t]{2}{*}{ Tide } & USGS & 01021060 & $\begin{array}{l}\text { St. Croix River at } \\
\text { Calais, ME }\end{array}$ & \multirow[t]{2}{*}{45.19166} & \multirow[t]{2}{*}{-67.28342} & \multirow[t]{2}{*}{$\begin{array}{c}\text { October } 2015 \\
\text { to present }\end{array}$} & \multirow{2}{*}{$\begin{array}{l}\text { Gage datum set to } \\
\text { NAVD } 88 . \text { Gage is } \\
\text { about } 6.1 \text { mi upstream } \\
\text { from ocean. }\end{array}$} & \multirow[t]{2}{*}{0.00} \\
\hline & & NOAA & 8410834 & $\begin{array}{l}\text { Pettegrove Point, } \\
\text { Dochet Island, } \\
\text { ME }\end{array}$ & & & & & \\
\hline 2 & Tide & NOAA & 8410140 & Eastport, ME & 44.90333 & -66.98500 & $\begin{array}{l}\text { September } \\
1929 \text { to } \\
\text { present }\end{array}$ & $\begin{array}{l}\text { Gage datum set to } \\
\text { MLLW. }\end{array}$ & -9.93 \\
\hline 3 & Tide & NOAA & 8411060 & $\begin{array}{l}\text { Cutler Farris } \\
\text { Wharf, ME }\end{array}$ & 44.65667 & -67.20500 & $\begin{array}{l}\text { September } \\
1963 \text { to } \\
\text { present }\end{array}$ & $\begin{array}{l}\text { Gage datum set to } \\
\text { MLLW. }\end{array}$ & -7.54 \\
\hline 4 & $\begin{array}{l}\text { Stream } \\
\text { gage } \\
\text { (stage- } \\
\text { only) }\end{array}$ & USGS & 01037050 & $\begin{array}{l}\text { Penobscot River at } \\
\text { Bangor, } \mathrm{ME}\end{array}$ & 44.79639 & -68.76778 & $\begin{array}{l}\text { December } \\
2009 \text { to } \\
\text { present }\end{array}$ & $\begin{array}{l}\text { Gage datum set to } \\
\text { NAVD } 88 \text {. Water } \\
\text { elevations likely af- } \\
\text { fected by river stage } \\
\text { as streamgage is about } \\
22.7 \text { mi upstream } \\
\text { from ocean. }\end{array}$ & 0.00 \\
\hline 5 & $\begin{array}{l}\text { Stream } \\
\text { gage } \\
\text { (stage- } \\
\text { only) }\end{array}$ & USGS & 01049320 & $\begin{array}{l}\text { Kennebec River at } \\
\text { Calumet Bridge } \\
\text { at Augusta, ME }\end{array}$ & 44.31861 & -69.77167 & $\begin{array}{c}\text { October } 1998 \\
\text { to present }\end{array}$ & $\begin{array}{l}\text { Gage datum is arbitrary. } \\
\text { Water elevations like- } \\
\text { ly affected by river } \\
\text { stage as streamgage } \\
\text { is about } 28.0 \text { mi up- } \\
\text { stream from ocean. }\end{array}$ & -2.01 \\
\hline 6 & $\begin{array}{l}\text { Stream } \\
\text { gage } \\
\text { (stage- } \\
\text { only) }\end{array}$ & USGS & 01049330 & $\begin{array}{c}\text { Kennebec River at } \\
\text { Hallowell, ME }\end{array}$ & 44.28681 & -69.78819 & $\begin{array}{l}\text { March } 2018 \text { to } \\
\text { present }\end{array}$ & $\begin{array}{l}\text { Gage datum set to } 1.30 \mathrm{ft} \\
\text { above NGVD } 29 . \\
\text { Water elevation con- } \\
\text { verted to NAVD } 88 \\
\text { using Vertcon. }{ }^{1} \text { Water } \\
\text { elevations likely af- } \\
\text { fected by river stage } \\
\text { as streamgage is about } \\
25.7 \text { mi upstream } \\
\text { from ocean. }\end{array}$ & 0.00 \\
\hline 7 & $\begin{array}{l}\text { Stream } \\
\text { gage } \\
\text { (stage- } \\
\text { only) }\end{array}$ & USGS & 01049505 & $\begin{array}{c}\text { Kennebec River at } \\
\text { Gardiner, ME }\end{array}$ & 44.23028 & -69.76944 & $\begin{array}{c}\text { February } 2000 \\
\text { to present }\end{array}$ & $\begin{array}{l}\text { Gage datum set to } \\
\text { NGVD } 29 \text {. Water } \\
\text { elevation converted } \\
\text { to NAVD } 88 \text { using } \\
\text { Vertcon. }{ }^{1} \text { Water eleva- } \\
\text { tions likely affected } \\
\text { by river stage as } \\
\text { streamgage is about } \\
21.5 \text { mi upstream } \\
\text { from ocean. }\end{array}$ & -0.69 \\
\hline
\end{tabular}


Table 1. Description of tide gages and coastal streamgages affected tidally or by backwater from tides with total water level data during the January and March 2018 nor'easters in New England.—Continued

[Station numbers (nos.) and locations of tide gages and coastal streamgages affected tidally or by backwater from tides are shown on figure 1. Tide gages operated by the U.S. Geological Survey (USGS) and the U.S. Army Corps of Engineers (USACE) list the paired National Oceanic and Atmospheric Administration (NOAA) tide gage for which predicted tidal data are available on the line below each of those tide gages. NOAA tide gages use the same gage for predicted tide data. USGS streamgages do not have a paired NOAA tide gage. Data for USGS tide gages and coastal streamgages are from U.S. Geological Survey (2019d); for NOAA tide gages, from National Oceanic and Atmospheric Administration (2019e); and for USACE tide gages, from U.S. Army Corps of Engineers (2019). ft, foot; NAVD 88, North American Vertical Datum of 1988; NGVD 29, National Geodetic Vertical Datum of 1929; MLLW, mean lower-low water; mi, mile; CT, Connecticut; MA, Massachusetts; ME, Maine; NH, New Hampshire; RI, Rhode Island; Rt., Route; St., Street; —, no number]

\begin{tabular}{|c|c|c|c|c|c|c|c|c|c|}
\hline $\begin{array}{l}\text { Stat } \\
\text { ion } \\
\text { no. }\end{array}$ & Gage type & $\begin{array}{l}\text { Agency } \\
\text { operat- } \\
\text { ing gage }\end{array}$ & $\begin{array}{c}\text { USGS or NOAA } \\
\text { station no. }\end{array}$ & Station name & $\begin{array}{l}\text { Latitude, } \\
\text { in decimal } \\
\text { degrees }\end{array}$ & $\begin{array}{c}\text { Longitude, } \\
\text { in decimal } \\
\text { degrees }\end{array}$ & $\begin{array}{l}\text { Period of } \\
\text { record }\end{array}$ & Remarks & $\begin{array}{c}\text { Conver } \\
\text { sion from } \\
\text { gage } \\
\text { datum to } \\
\text { NAVD 88, } \\
\text { in ft }\end{array}$ \\
\hline \multicolumn{10}{|c|}{ Maine, sites from Portland south } \\
\hline 8 & Tide & NOAA & 8418150 & Portland, ME & 43.65833 & -70.24500 & $\begin{array}{l}\text { March } 1910 \text { to } \\
\text { present }\end{array}$ & $\begin{array}{l}\text { Gage datum set to } \\
\text { MLLW. }\end{array}$ & -5.26 \\
\hline \multirow[t]{2}{*}{9} & Tide & USGS & 432742070225401 & $\begin{array}{l}\text { Saco River at } \\
\text { Camp Ellis near } \\
\text { Saco, Maine }\end{array}$ & 43.46163 & -70.38170 & $\begin{array}{l}\text { April } 2016 \text { to } \\
\text { present }\end{array}$ & $\begin{array}{l}\text { Gage datum set to } \\
\text { NAVD } 88 \text {. }\end{array}$ & 0.00 \\
\hline & & NOAA & 8418606 & $\begin{array}{l}\text { Camp Ellis, Saco } \\
\text { River, ME }\end{array}$ & & & & & \\
\hline 10 & Tide & NOAA & 8419317 & Wells, ME & 43.32000 & -70.56333 & $\begin{array}{l}\text { June } 1999 \text { to } \\
\text { present }\end{array}$ & $\begin{array}{l}\text { Gage datum set to } \\
\text { MLLW. }\end{array}$ & -5.14 \\
\hline \multicolumn{10}{|c|}{ Massachusetts, sites on eastern coastline and Cape Cod Bay } \\
\hline 11 & $\begin{array}{l}\text { Stream } \\
\text { gage } \\
\text { (stage- } \\
\text { only) }\end{array}$ & USGS & 01100693 & $\begin{array}{l}\text { Merrimack River, } \\
0.3 \text { mi upstream } \\
\text { Rt. } 125 \text {, at } \\
\text { Haverhill, MA }\end{array}$ & 42.77278 & -71.08306 & $\begin{array}{l}\text { July } 2006 \text { to } \\
\text { present }\end{array}$ & $\begin{array}{l}\text { Gage datum is arbitrary. } \\
\text { Water elevations like- } \\
\text { ly affected by river } \\
\text { stage as streamgage } \\
\text { is about } 15.5 \text { mi up- } \\
\text { stream from ocean. }\end{array}$ & -1.17 \\
\hline \multirow[t]{2}{*}{12} & Tide & USGS & 01100870 & $\begin{array}{l}\text { Merrimack River } \\
\text { at Newburyport, } \\
\text { MA }\end{array}$ & 42.81565 & -70.87283 & $\begin{array}{c}\text { January } 2015 \\
\text { to present }\end{array}$ & $\begin{array}{l}\text { Gage datum set to } \\
\text { NAVD } 88 .\end{array}$ & 0.00 \\
\hline & & NOAA & 8440466 & $\begin{array}{c}\text { Newburyport, } \\
\text { Merrimack } \\
\text { River, MA }\end{array}$ & & & & & \\
\hline 13 & $\begin{array}{r}\text { Stream } \\
\text { gage }\end{array}$ & USGS & 01102345 & $\begin{array}{l}\text { Saugus River } \\
\text { at Saugus } \\
\text { Ironworks at } \\
\text { Saugus, MA }\end{array}$ & 42.46954 & -71.00700 & $\begin{array}{l}\text { March } 1994 \text { to } \\
\text { present }\end{array}$ & $\begin{array}{l}\text { Gage datum is arbitrary. } \\
\text { Water elevations like- } \\
\text { ly affected by river } \\
\text { stage as streamgage is } \\
\text { about } 2.3 \text { mi upstream } \\
\text { from ocean. }\end{array}$ & 5.58 \\
\hline \multirow[t]{2}{*}{14} & Tide & USGS & 01103050 & $\begin{array}{l}\text { Mystic River at } \\
\text { Amelia Earhart } \\
\text { Dam near } \\
\text { Somerville, MA }\end{array}$ & 42.39565 & -71.07505 & $\begin{array}{r}\text { December } \\
2007 \text { to } \\
\text { present }\end{array}$ & \multirow{2}{*}{$\begin{array}{l}\text { Gage datum set to } \\
\text { Boston City Base } \\
\text { datum. Water eleva- } \\
\text { tions from ocean side } \\
\text { (downstream side) of } \\
\text { locks. }\end{array}$} & \multirow[t]{2}{*}{-106.68} \\
\hline & & NOAA & 8443662 & $\begin{array}{c}\text { Amelia Earhart } \\
\text { Dam, Mystic } \\
\text { River, MA }\end{array}$ & & & & & \\
\hline \multirow[t]{2}{*}{15} & Tide & USGS & 01104715 & $\begin{array}{l}\text { Charles River, new } \\
\text { Charles River } \\
\text { Dam at Boston, } \\
\text { MA }\end{array}$ & 42.36889 & -71.06167 & $\begin{array}{l}\text { September } \\
2002 \text { to } \\
\text { present }\end{array}$ & \multirow{2}{*}{$\begin{array}{l}\text { Gage datum set to } \\
\text { Boston City Base } \\
\text { datum. Water eleva- } \\
\text { tions from ocean side } \\
\text { (downstream side) of } \\
\text { locks. }\end{array}$} & \multirow[t]{2}{*}{-106.71} \\
\hline & & NOAA & 8443838 & $\begin{array}{l}\text { Charlestown, } \\
\text { Charles River } \\
\text { entrance, MA }\end{array}$ & & & & & \\
\hline 16 & Tide & NOAA & 8443970 & Boston, MA & 42.35333 & -71.05000 & $\begin{array}{l}\text { May } 1921 \text { to } \\
\text { present }\end{array}$ & $\begin{array}{l}\text { Gage datum set to } \\
\text { MLLW. }\end{array}$ & -5.51 \\
\hline
\end{tabular}


Table 1. Description of tide gages and coastal streamgages affected tidally or by backwater from tides with total water level data during the January and March 2018 nor'easters in New England.-Continued

[Station numbers (nos.) and locations of tide gages and coastal streamgages affected tidally or by backwater from tides are shown on figure 1. Tide gages operated by the U.S. Geological Survey (USGS) and the U.S. Army Corps of Engineers (USACE) list the paired National Oceanic and Atmospheric Administration (NOAA) tide gage for which predicted tidal data are available on the line below each of those tide gages. NOAA tide gages use the same gage for predicted tide data. USGS streamgages do not have a paired NOAA tide gage. Data for USGS tide gages and coastal streamgages are from U.S. Geological Survey (2019d); for NOAA tide gages, from National Oceanic and Atmospheric Administration (2019e); and for USACE tide gages, from U.S. Army Corps of Engineers (2019). ft, foot; NAVD 88, North American Vertical Datum of 1988; NGVD 29, National Geodetic Vertical Datum of 1929; MLLW, mean lower-low water; mi, mile; CT, Connecticut; MA, Massachusetts; ME, Maine; NH, New Hampshire; RI, Rhode Island; Rt., Route; St., Street; —, no number]

\begin{tabular}{|c|c|c|c|c|c|c|c|c|c|}
\hline $\begin{array}{l}\text { Stat } \\
\text { ion } \\
\text { no. }\end{array}$ & Gage type & $\begin{array}{l}\text { Agency } \\
\text { operat- } \\
\text { ing gage }\end{array}$ & $\begin{array}{l}\text { USGS or NOAA } \\
\text { station no. }\end{array}$ & Station name & $\begin{array}{l}\text { Latitude, } \\
\text { in decimal } \\
\text { degrees }\end{array}$ & $\begin{array}{c}\text { Longitude, } \\
\text { in decimal } \\
\text { degrees }\end{array}$ & $\begin{array}{l}\text { Period of } \\
\text { record }\end{array}$ & Remarks & $\begin{array}{c}\text { Conver } \\
\text { sion from } \\
\text { gage } \\
\text { datum to } \\
\text { NAVD 88, } \\
\text { in } \mathrm{ft}\end{array}$ \\
\hline \multicolumn{10}{|c|}{ Massachusetts, sites on eastern coastline and Cape Cod Bay—Continued } \\
\hline 17 & $\begin{array}{l}\text { Stream } \\
\text { gage }\end{array}$ & USGS & 011055566 & $\begin{array}{l}\text { Neponset River at } \\
\text { Milton Village, } \\
\text { MA }\end{array}$ & 42.27093 & -71.06838 & $\begin{array}{r}\text { November } \\
1996 \text { to } \\
\text { present }\end{array}$ & $\begin{array}{l}\text { Gage datum is arbitrary. } \\
\text { Water elevations like- } \\
\text { ly affected by river } \\
\text { stage as streamgage is } \\
\text { about } 4.1 \text { mi upstream } \\
\text { from ocean. }\end{array}$ & 2.96 \\
\hline 18 & $\begin{array}{l}\text { Stream } \\
\text { gage }\end{array}$ & USGS & 01105585 & $\begin{array}{r}\text { Town Brook at } \\
\text { Quincy, MA }\end{array}$ & 42.24788 & -70.99727 & $\begin{array}{c}\text { October } 1998 \\
\text { to present }\end{array}$ & $\begin{array}{l}\text { Gage datum is arbitrary. } \\
\text { Water elevations like- } \\
\text { ly affected by river } \\
\text { stage as streamgage is } \\
\text { about } 0.8 \text { mi upstream } \\
\text { from ocean. }\end{array}$ & 4.53 \\
\hline 19 & $\begin{array}{l}\text { Stream } \\
\text { gage }\end{array}$ & USGS & 01105583 & $\begin{array}{l}\text { Monatiquot } \\
\text { River at East } \\
\text { Braintree, MA }\end{array}$ & 42.22093 & -70.97810 & $\begin{array}{l}\text { March } 2006 \text { to } \\
\text { present }\end{array}$ & $\begin{array}{l}\text { Gage datum is arbitrary. } \\
\text { Water elevations like- } \\
\text { ly affected by river } \\
\text { stage as streamgage is } \\
\text { about } 2.8 \text { mi upstream } \\
\text { from ocean. }\end{array}$ & 5.18 \\
\hline 20 & $\begin{array}{l}\text { Stream } \\
\text { gage }\end{array}$ & USGS & 01105730 & $\begin{array}{r}\text { Indian Head River } \\
\text { at Hanover, MA }\end{array}$ & 42.10066 & -70.82254 & $\begin{array}{l}\text { July } 1966 \text { to } \\
\text { present }\end{array}$ & $\begin{array}{l}\text { Gage datum is arbitrary. } \\
\text { Water elevations like- } \\
\text { ly affected by river } \\
\text { stage as streamgage } \\
\text { is about } 11.2 \text { mi up- } \\
\text { stream from ocean. }\end{array}$ & 2.07 \\
\hline 21 & $\begin{array}{l}\text { Stream } \\
\text { gage }\end{array}$ & USGS & 01105870 & $\begin{array}{l}\text { Jones River at } \\
\text { Kingston, MA }\end{array}$ & 41.99094 & -70.73365 & $\begin{array}{r}\text { August } 1966 \\
\text { to present }\end{array}$ & $\begin{array}{l}\text { Gage datum is arbitrary. } \\
\text { Water elevations like- } \\
\text { ly affected by river } \\
\text { stage as streamgage is } \\
\text { about } 2.1 \text { mi upstream } \\
\text { from ocean. }\end{array}$ & 3.88 \\
\hline 22 & $\begin{array}{l}\text { Stream } \\
\text { gage }\end{array}$ & USGS & 01105876 & $\begin{array}{l}\text { Eel River at Rt. 3A } \\
\text { near Plymouth, } \\
\text { MA }\end{array}$ & 41.94177 & -70.62253 & $\begin{array}{l}\text { December } \\
1969 \text { to } \\
\text { Septem- } \\
\text { ber } 1971 \\
\text { June } 2006 \\
\text { to present }\end{array}$ & $\begin{array}{l}\text { Gage datum is arbitrary. } \\
\text { Water elevations like- } \\
\text { ly affected by river } \\
\text { stage as streamgage is } \\
\text { about } 0.6 \text { mi upstream } \\
\text { from ocean. }\end{array}$ & 2.11 \\
\hline \multirow[t]{2}{*}{23} & Tide & USGS & 414507070091400 & $\begin{array}{l}\text { Sesuit Harbor Tide } \\
\text { Gage at Dennis, } \\
\text { MA }\end{array}$ & 41.75194 & -70.15389 & $\begin{array}{c}\text { January } 2015 \\
\text { to present }\end{array}$ & $\begin{array}{l}\text { Gage datum set to } \\
\text { NAVD } 88 \text {. }\end{array}$ & 0.00 \\
\hline & & NOAA & 8447241 & $\begin{array}{l}\text { Sesuit Harbor, East } \\
\text { Dennis, MA }\end{array}$ & & & & & \\
\hline
\end{tabular}


Table 1. Description of tide gages and coastal streamgages affected tidally or by backwater from tides with total water level data during the January and March 2018 nor'easters in New England. - Continued

[Station numbers (nos.) and locations of tide gages and coastal streamgages affected tidally or by backwater from tides are shown on figure 1. Tide gages operated by the U.S. Geological Survey (USGS) and the U.S. Army Corps of Engineers (USACE) list the paired National Oceanic and Atmospheric Administration (NOAA) tide gage for which predicted tidal data are available on the line below each of those tide gages. NOAA tide gages use the same gage for predicted tide data. USGS streamgages do not have a paired NOAA tide gage. Data for USGS tide gages and coastal streamgages are from U.S. Geological Survey (2019d); for NOAA tide gages, from National Oceanic and Atmospheric Administration (2019e); and for USACE tide gages, from U.S. Army Corps of Engineers (2019). ft, foot; NAVD 88, North American Vertical Datum of 1988; NGVD 29, National Geodetic Vertical Datum of 1929; MLLW, mean lower-low water; mi, mile; CT, Connecticut; MA, Massachusetts; ME, Maine; NH, New Hampshire; RI, Rhode Island; Rt., Route; St., Street; —, no number]

\begin{tabular}{|c|c|c|c|c|c|c|c|c|c|}
\hline $\begin{array}{l}\text { Stat } \\
\text { ion } \\
\text { no. }\end{array}$ & Gage type & $\begin{array}{l}\text { Agency } \\
\text { operat- } \\
\text { ing gage }\end{array}$ & $\begin{array}{l}\text { USGS or NOAA } \\
\text { station no. }\end{array}$ & Station name & $\begin{array}{l}\text { Latitude, } \\
\text { in decimal } \\
\text { degrees }\end{array}$ & $\begin{array}{c}\text { Longitude, } \\
\text { in decimal } \\
\text { degrees }\end{array}$ & $\begin{array}{l}\text { Period of } \\
\text { record }\end{array}$ & Remarks & $\begin{array}{c}\text { Conver } \\
\text { sion from } \\
\text { gage } \\
\text { datum to } \\
\text { NAVD 88, } \\
\text { in ft }\end{array}$ \\
\hline \multicolumn{10}{|c|}{ Massachusetts, sites on eastern coastline and Cape Cod Bay-Continued } \\
\hline \multirow[t]{2}{*}{24} & Tide & USGS & 011058798 & $\begin{array}{l}\text { Herring River at } \\
\text { Chequessett } \\
\text { Neck Road at } \\
\text { Wellfleet, MA }\end{array}$ & 41.59233 & -70.50781 & $\begin{array}{l}\text { September } \\
2017 \text { to } \\
\text { present }\end{array}$ & $\begin{array}{l}\text { Gage datum set to } \\
\text { NAVD 88. Ocean side } \\
\text { (downstream side) of } \\
\text { culvert and gates. }\end{array}$ & 0.00 \\
\hline & & NOAA & 8446613 & Wellfleet, MA & & & & & \\
\hline \multirow[t]{2}{*}{25} & Tide & USGS & 420259070105600 & $\begin{array}{l}\text { Provincetown } \\
\text { Tide Gage, } \\
\text { Provincetown, } \\
\text { MA }\end{array}$ & 42.04972 & -70.18222 & $\begin{array}{r}\text { December } \\
2014 \text { to } \\
\text { present }\end{array}$ & $\begin{array}{l}\text { Gage datum set to } \\
\text { NAVD } 88 \text {. }\end{array}$ & 0.00 \\
\hline & & NOAA & 8446121 & Provincetown, MA & & & & & \\
\hline \multicolumn{10}{|c|}{ Massachusetts, sites on southern coastline } \\
\hline \multirow[t]{2}{*}{26} & Tide & USGS & 413601070275800 & $\begin{array}{l}\text { Popponesset Bay, } \\
\text { Mashpee Neck } \\
\text { Road, near } \\
\text { Mashpee, MA }\end{array}$ & 41.60016 & -70.46612 & $\begin{array}{c}\text { June } 2014 \text { to } \\
\text { present }\end{array}$ & $\begin{array}{l}\text { Gage datum set to } \\
\text { NAVD } 88 \text {. }\end{array}$ & 0.00 \\
\hline & & NOAA & 8447742 & $\begin{array}{l}\text { Popponesset } \\
\text { Island, } \\
\text { Popponesset } \\
\text { Bay, MA }\end{array}$ & & & & & \\
\hline 27 & $\begin{array}{l}\text { Stream } \\
\text { gage }\end{array}$ & USGS & 011058837 & $\begin{array}{c}\text { Quashnet River } \\
\text { at Waquiot } \\
\text { Village, MA }\end{array}$ & 41.93111 & -70.06456 & $\begin{array}{c}\text { October } 1988 \\
\text { to present }\end{array}$ & $\begin{array}{l}\text { Gage datum is arbitrary. } \\
\text { Water elevations like- } \\
\text { ly affected by river } \\
\text { stage as streamgage is } \\
\text { about } 2.1 \text { mi upstream } \\
\text { from ocean. }\end{array}$ & -0.46 \\
\hline 28 & Tide & NOAA & 8447930 & Woods Hole, MA & 41.52333 & -70.67167 & $\begin{array}{l}\text { July } 1932 \text { to } \\
\text { present }\end{array}$ & $\begin{array}{l}\text { Gage datum set to } \\
\text { MLLW. }\end{array}$ & -1.36 \\
\hline \multirow[t]{2}{*}{29} & Tide & USACE & - & $\begin{array}{l}\text { New Bedford } \\
\text { Hurricane } \\
\text { Barrier, New } \\
\text { Bedford, MA }\end{array}$ & 41.62431 & -70.90587 & - & $\begin{array}{l}\text { Gage datum set to } \\
\text { NGVD 29. Water } \\
\text { elevation converted } \\
\text { to NAVD } 88 \text { using }\end{array}$ & -0.83 \\
\hline & & NOAA & 8447584 & $\begin{array}{l}\text { New Bedford } \\
\text { Bridge, MA }\end{array}$ & & & & Vertcon. ${ }^{1}$ & \\
\hline 30 & Tide & NOAA & 8447386 & Fall River, MA & 41.70333 & -71.16333 & $\begin{array}{c}\text { October } 1955 \\
\text { to present }\end{array}$ & $\begin{array}{l}\text { Gage datum set to } \\
\text { MLLW. }\end{array}$ & -2.41 \\
\hline
\end{tabular}


Table 1. Description of tide gages and coastal streamgages affected tidally or by backwater from tides with total water level data during the January and March 2018 nor'easters in New England.-Continued

[Station numbers (nos.) and locations of tide gages and coastal streamgages affected tidally or by backwater from tides are shown on figure 1. Tide gages operated by the U.S. Geological Survey (USGS) and the U.S. Army Corps of Engineers (USACE) list the paired National Oceanic and Atmospheric Administration (NOAA) tide gage for which predicted tidal data are available on the line below each of those tide gages. NOAA tide gages use the same gage for predicted tide data. USGS streamgages do not have a paired NOAA tide gage. Data for USGS tide gages and coastal streamgages are from U.S. Geological Survey (2019d); for NOAA tide gages, from National Oceanic and Atmospheric Administration (2019e); and for USACE tide gages, from U.S. Army Corps of Engineers (2019). ft, foot; NAVD 88, North American Vertical Datum of 1988; NGVD 29, National Geodetic Vertical Datum of 1929; MLLW, mean lower-low water; mi, mile; CT, Connecticut; MA, Massachusetts; ME, Maine; NH, New Hampshire; RI, Rhode Island; Rt., Route; St., Street; —, no number]

\begin{tabular}{|c|c|c|c|c|c|c|c|c|c|}
\hline $\begin{array}{l}\text { Stat } \\
\text { ion } \\
\text { no. }\end{array}$ & Gage type & $\begin{array}{l}\text { Agency } \\
\text { operat- } \\
\text { ing gage }\end{array}$ & $\begin{array}{l}\text { USGS or NOAA } \\
\text { station no. }\end{array}$ & Station name & $\begin{array}{l}\text { Latitude, } \\
\text { in decimal } \\
\text { degrees }\end{array}$ & $\begin{array}{l}\text { Longitude, } \\
\text { in decimal } \\
\text { degrees }\end{array}$ & $\begin{array}{l}\text { Period of } \\
\text { record }\end{array}$ & Remarks & $\begin{array}{l}\text { Conver } \\
\text { sion from } \\
\text { gage } \\
\text { datum to } \\
\text { NAVD 88, } \\
\text { in } \mathrm{ft}\end{array}$ \\
\hline \multicolumn{10}{|c|}{ Rhode Island } \\
\hline 31 & Tide & NOAA & 8452660 & Newport, RI & 41.50333 & -71.32667 & $\begin{array}{l}\text { September } \\
1930 \text { to } \\
\text { present }\end{array}$ & $\begin{array}{l}\text { Gage datum set to } \\
\text { MLLW. }\end{array}$ & -2.04 \\
\hline 32 & Tide & NOAA & 8454000 & Providence, RI & 41.80667 & -71.40000 & $\begin{array}{l}\text { June } 1938 \text { to } \\
\text { present }\end{array}$ & $\begin{array}{l}\text { Gage datum set to } \\
\text { MLLW. }\end{array}$ & -2.47 \\
\hline 33 & $\begin{array}{l}\text { Stream } \\
\text { gage }\end{array}$ & USGS & 01118500 & $\begin{array}{l}\text { Pawcatuck River } \\
\text { at Westerly, RI }\end{array}$ & 41.38371 & -71.83312 & $\begin{array}{l}\text { November } \\
1940 \text { to } \\
\text { present }\end{array}$ & $\begin{array}{l}\text { Gage datum is arbitrary. } \\
\text { Water elevations like- } \\
\text { ly affected by river } \\
\text { stage as streamgage is } \\
\text { about } 4.5 \text { mi upstream } \\
\text { from ocean. }\end{array}$ & -2.68 \\
\hline \multirow[t]{2}{*}{34} & Tide & USGS & 411838071513000 & $\begin{array}{l}\text { Watch Hill Cove } \\
\text { Tide Gage } \\
\text { Westerly, RI }\end{array}$ & 41.31056 & -71.85833 & $\begin{array}{r}\text { August } 2014 \\
\text { to present }\end{array}$ & $\begin{array}{l}\text { Gage datum set to } \\
\text { NAVD } 88 .\end{array}$ & 0.00 \\
\hline & & NOAA & 8458694 & $\begin{array}{l}\text { Watch Hill Point, } \\
\text { RI }\end{array}$ & & & & & \\
\hline \multicolumn{10}{|c|}{ Connecticut } \\
\hline 35 & Tide & NOAA & 8461490 & $\begin{array}{l}\text { New London, } \\
\text { Thames River, } \\
\text { CT }\end{array}$ & 41.35500 & -72.08667 & $\begin{array}{l}\text { June } 1938 \text { to } \\
\text { present }\end{array}$ & $\begin{array}{l}\text { Gage datum set to } \\
\text { MLLW. }\end{array}$ & -1.84 \\
\hline \multirow[t]{2}{*}{36} & Tide & USGS & 01194750 & $\begin{array}{l}\text { Connecticut River } \\
\text { at Essex, CT }\end{array}$ & 41.35148 & -72.38437 & $\begin{array}{c}\text { February } 2010 \\
\text { to present }\end{array}$ & $\begin{array}{l}\text { Gage datum set to } \\
\text { NGVD 29. Water }\end{array}$ & -1.02 \\
\hline & & NOAA & 8462925 & Essex, CT & & & & $\begin{array}{l}\text { elevation converted } \\
\text { to NAVD } 88 \text { using } \\
\text { Vertcon. }{ }^{1} \text { Gage is } \\
\text { about } 3.6 \text { mi upstream } \\
\text { from ocean. }\end{array}$ & \\
\hline \multirow[t]{2}{*}{37} & Tide & USGS & 01194796 & $\begin{array}{l}\text { Connecticut River } \\
\text { at Old Lyme, } \\
\text { CT }\end{array}$ & 41.31260 & -72.34592 & $\begin{array}{c}\text { October } 2017 \\
\text { to present }\end{array}$ & $\begin{array}{l}\text { Gage datum set to } \\
\text { NGVD 29. Water } \\
\text { elevation converted to }\end{array}$ & -1.01 \\
\hline & & NOAA & 8462764 & $\begin{array}{l}\text { Lyme, highway } \\
\text { bridge, CT }\end{array}$ & & & & NAVD 88 from levels. & \\
\hline \multirow[t]{2}{*}{38} & Tide & USGS & 01194815 & $\begin{array}{l}\text { Connecticut River } \\
\text { near Bridge } \\
\text { St. near Old } \\
\text { Saybrook, CT }\end{array}$ & 41.28133 & -72.34928 & $\begin{array}{l}\text { September } \\
2015 \text { to } \\
\text { present }\end{array}$ & $\begin{array}{l}\text { Gage datum set to } \\
\text { NAVD } 88 \text {. }\end{array}$ & 0.00 \\
\hline & & NOAA & 8462752 & $\begin{array}{l}\text { Saybrook Point, } \\
\text { CT }\end{array}$ & & & & & \\
\hline 39 & Tide & NOAA & 8467150 & Bridgeport, CT & 41.17500 & -73.18333 & $\begin{array}{l}\text { June } 1932 \text { to } \\
\text { present }\end{array}$ & $\begin{array}{l}\text { Gage datum set to } \\
\text { MLLW. }\end{array}$ & -3.84 \\
\hline
\end{tabular}


Table 1. Description of tide gages and coastal streamgages affected tidally or by backwater from tides with total water level data during the January and March 2018 nor'easters in New England.—Continued

[Station numbers (nos.) and locations of tide gages and coastal streamgages affected tidally or by backwater from tides are shown on figure 1. Tide gages operated by the U.S. Geological Survey (USGS) and the U.S. Army Corps of Engineers (USACE) list the paired National Oceanic and Atmospheric Administration (NOAA) tide gage for which predicted tidal data are available on the line below each of those tide gages. NOAA tide gages use the same gage for predicted tide data. USGS streamgages do not have a paired NOAA tide gage. Data for USGS tide gages and coastal streamgages are from U.S. Geological Survey (2019d); for NOAA tide gages, from National Oceanic and Atmospheric Administration (2019e); and for USACE tide gages, from U.S. Army Corps of Engineers (2019). ft, foot; NAVD 88, North American Vertical Datum of 1988; NGVD 29, National Geodetic Vertical Datum of 1929; MLLW, mean lower-low water; mi, mile; CT, Connecticut; MA, Massachusetts; ME, Maine; NH, New Hampshire; RI, Rhode Island; Rt., Route; St., Street; —, no number]

\begin{tabular}{|c|c|c|c|c|c|c|c|c|c|}
\hline $\begin{array}{l}\text { Stat } \\
\text { ion } \\
\text { no. }\end{array}$ & Gage type & $\begin{array}{l}\text { Agency } \\
\text { operat- } \\
\text { ing gage }\end{array}$ & $\begin{array}{l}\text { USGS or NOAA } \\
\text { station no. }\end{array}$ & Station name & $\begin{array}{l}\text { Latitude, } \\
\text { in decimal } \\
\text { degrees }\end{array}$ & $\begin{array}{c}\text { Longitude, } \\
\text { in decimal } \\
\text { degrees }\end{array}$ & $\begin{array}{l}\text { Period of } \\
\text { record }\end{array}$ & Remarks & $\begin{array}{c}\text { Conver } \\
\text { sion from } \\
\text { gage } \\
\text { datum to } \\
\text { NAVD 88, } \\
\text { in ft }\end{array}$ \\
\hline \multicolumn{10}{|c|}{ Connecticut-Continued } \\
\hline \multirow[t]{2}{*}{40} & Tide & USGS & 01209510 & $\begin{array}{l}\text { Saugatuck River } \\
\text { at Rt. } 1 \text { at } \\
\text { Westport, CT }\end{array}$ & 41.14089 & -73.36303 & $\begin{array}{c}\text { January } 2014 \\
\text { to present }\end{array}$ & \multirow{2}{*}{$\begin{array}{l}\text { Gage datum set to } \\
\text { NAVD } 88 . \text { Gage is } \\
\text { about } 1.6 \text { mi upstream } \\
\text { from ocean. }\end{array}$} & \multirow[t]{2}{*}{0.00} \\
\hline & & NOAA & 8468191 & Saugatuck River & & & & & \\
\hline \multirow[t]{2}{*}{41} & Tide & USACE & - & $\begin{array}{l}\text { Stamford } \\
\quad \text { Hurricane } \\
\text { Barrier, } \\
\text { Stamford, CT }\end{array}$ & 41.03694 & -73.53472 & - & \multirow{2}{*}{$\begin{array}{l}\text { Gage datum set to } \\
\text { NGVD } 29 . \text { Water } \\
\text { elevation converted } \\
\text { to NAVD } 88 \text { using } \\
\text { Vertcon. }{ }^{1} \text { Gage is } \\
\text { about } 3.6 \text { mi upstream } \\
\text { from ocean. }\end{array}$} & \multirow[t]{2}{*}{-0.90} \\
\hline & & NOAA & 8469198 & $\begin{array}{l}\text { Stamford Harbor, } \\
\text { CT }\end{array}$ & & & & & \\
\hline \multirow[t]{2}{*}{42} & Tide & USGS & 012112296 & $\begin{array}{l}\text { Greenwich Harbor } \\
\text { at Grass Island } \\
\text { at Greenwich, } \\
\text { CT }\end{array}$ & 41.01412 & -73.62508 & $\begin{array}{c}\text { January } 2015 \\
\text { to present }\end{array}$ & \multirow[t]{2}{*}{$\begin{array}{l}\text { Gage datum set to } \\
\text { NAVD } 88 \text {. }\end{array}$} & \multirow[t]{2}{*}{0.00} \\
\hline & & NOAA & 8469549 & $\begin{array}{l}\text { Cos Cob Harbor, } \\
\text { CT }\end{array}$ & & & & & \\
\hline
\end{tabular}

\footnotetext{
1Vertcon is an online tool for converting NGVD 29 elevations to NAVD 88 elevation (National Oceanic and Atmospheric Administration, 2020).
} 
Table 2. Description of U.S. Geological Survey temporary water-level sensors deployed during the March 2-4, 2018, nor'easter in New England.

[Station numbers (nos.) and locations of U.S. Geological Survey (USGS) temporary water-level sensors shown on figure 1. STN, short-term network; St., Street; Rd., Road; Rt., Route; Ln., Lane; Dr., Drive; CT, Connecticut; MA, Massachusetts; ME, Maine; NH, New Hampshire]




Table 2. Description of U.S. Geological Survey temporary water-level sensors deployed during the March 2-4, 2018, nor'easter in New England.—Continued

[Station numbers (nos.) and locations of U.S. Geological Survey (USGS) temporary water-level sensors shown on figure 1. STN, short-term network; St., Street; Rd., Road; Rt., Route; Ln., Lane; Dr., Drive; CT, Connecticut; MA, Massachusetts; ME, Maine; NH, New Hampshire]

\begin{tabular}{cllcc}
\hline $\begin{array}{c}\text { Station } \\
\text { no. }\end{array}$ & USGS STN no. & \multicolumn{1}{c}{ USGS STN site name } & $\begin{array}{c}\text { Latitude, } \\
\text { in decimal } \\
\text { degrees }\end{array}$ & $\begin{array}{c}\text { Longitude, } \\
\text { in decimal } \\
\text { degrees }\end{array}$ \\
\hline & & & Connecticut (sites listed east to west) -Continued & \\
\hline 70 & CTMID04661 & Back River, downstream of Rt. 154 bridge, Old Saybrook, CT & 41.27639 \\
\hline 71 & CTMID04673 & Clinton Harbor, Cedar Island Marina, Riverside Dr., Clinton, CT & 41.26861 & -72.39333 \\
72 & CTNEW04687 & East River, boat launch at end of Old Post Rd., Madison, CT & 41.28674 & -72.64608 \\
73 & CTNEW04662 & West River, downstream side of Rt. 146 bridge, Guilford, CT & 41.27904 & -72.68790 \\
\hline 74 & CTNEW04671 & Branford Point Pier, end of Harbor St., Branford, CT & 41.26038 & -72.82070 \\
75 & CTNEW04676 & Cosey Beach, Caroline Rd., East Haven, CT & 41.24310 & -72.88250 \\
76 & CTFAI04670 & Norwalk River, Norwalk Maritime Aquarium public dock, Fairfield, CT & 41.10257 & -73.41644 \\
77 & CTFAI15448 & Public dock on Grass Island Rd., Belle Haven, Greenwich, CT & 41.01412 & -73.62508 \\
\hline
\end{tabular}

Depending on the location of the HWM found, the field personnel flagged the mark using one or more of the following: USGS HWM disk, paint, permanent marker, nail, PK nail, chiseled mark, stake, or surveyor's hub. Photographs of the HWMs (fig. 5) and the pathways to the HWM were taken; photographs of the surrounding area were also taken if needed. To provide additional guidance for relocating and recovering the HWMs, coordinates obtained by a handheld Global Positioning System (GPS) device (usually a cell phone) were recorded on the field form.

Field crews identified and flagged a total of $86 \mathrm{HWMs}$ in Massachusetts following the January 2018 nor'easter (fig. 3; U.S. Geological Survey, 2019b). Following the March 2018 nor'easter, field crews identified and flagged a total of 115 HWMs: 86 sites in Massachusetts, 2 sites in Rhode Island, 13 sites in New Hampshire, 6 sites in Maine, and 8 sites in Connecticut (fig. 4; U.S. Geological Survey, 2019c). At 52 of the 86 sites in Massachusetts, HWMs were collected during both the January and March 2018 nor'easters.

\section{Surveying}

Positions of the coastal HWMs from the January and March 2018 nor'easters were surveyed using Global Navigation Satellite System (GNSS) methods described by Rydlund and Densmore (2012). Specifically, real-time network (RTN) positioning was used, which provided centimeter-level $(0.03-\mathrm{ft})$ vertical precision through continuous real-time differential corrections between GNSS reference stations and the receiver. Trimble R8 and Topcon Hyper V GNSS receivers were used for RTN surveys. KeyNetGPS, Inc. (2018) GNSS reference network stations provided differential corrections to position data. Peak total water level elevations surveyed were collected in the North American Vertical Datum of 1988 (NAVD 88). Horizontal positions surveyed were collected in the North American Datum (NAD 83) State Plane Coordinate System. Where overhead vegetation or structures interfered with satellite reception or when known reference marks existed nearby at USGS temporary water-level sensor brackets and National Geodetic Survey (NGS) or Massachusetts Department of Transportation (MassDOT) benchmarks, HWM elevations were measured with an electronic digital level, following methods described by Kenney (2010).

RTN and leveling of HWM elevations provided surveying results to meet USGS GNSS level II precision standards (Rydlund and Densmore, 2012). A few HWMs surveyed had more uncertainty in the elevations; the flagged elevations for 3 of 10 missing HWMs were recreated using notes and photographs from the original surveys. Hand measurements illustrating the heights of the HWMs above the ground provided references to survey elevations beneath the missing marks. Ground competency was considered when surveying reference points, and neither unconsolidated earth nor snow were used to reference missing HWM elevations.

Table 3. Uncertainty of high-water marks for coastal areas of New England.

[HWM, high-water mark; ft, foot; \pm , plus or minus; >, greater than]

\begin{tabular}{lc}
\hline \multicolumn{1}{c}{ HWM classification } & Vertical uncertainty in HWM, in $\mathbf{f t}^{\mathbf{1}}$ \\
\hline Excellent & \pm 0.05 \\
Good & \pm 0.10 \\
Fair & \pm 0.20 \\
Poor & \pm 0.40 \\
Very poor & $>0.40$ \\
\hline
\end{tabular}

1Data are from Rydlund and Densmore (2012) and Koenig and others (2016). 
For the entirety of the survey, quality control procedures from Rydlund and Densmore (2012) were followed to ensure accurate results. Network elevation accuracy was checked at nearby NGS and MassDOT benchmarks at the beginning and end of each survey day. Surveyed elevations at these benchmarks were compared with the published elevations maintained for those benchmarks. The accuracies of the NAVD 88 elevations were checked on benchmarks before and after each RTN campaign. Redundant, blunder checks were also measured at each benchmark (table 1.1). Blunder checks can expose multipathing errors that may occur with dualband receivers. Measurements within the $0.10-\mathrm{ft}$ tolerance set by the USGS GNSS level II quality standard (Rydlund and Densmore, 2012) were averaged for the final elevation. If the two measured elevations differed by more than $0.10 \mathrm{ft}$, then subsequent measurements were made to obtain at least two elevations that were within $0.10 \mathrm{ft}$ of each other. If a benchmark was in an area with obstructions or other reasons for poor satellite reception, then a temporary reference mark was set nearby where reception existed and a blunder checks could be done following the same standards. Then traditional levels were run between the benchmark and the temporary reference mark to compare the elevation to the published benchmark NAVD 88 elevation.

A network of continuous real-time differential corrected sites was provided by a fixed-base GPS network of sites under the operation of KeyNetGPS, Inc. (2018). The measurement accuracy of the GPS elevations was checked by comparing GPS readings at primarily NGS and MassDOT benchmarks with established NAVD 88 elevations near the coasts of New Hampshire, Massachusetts, and Rhode Island during August to November 2018 and February 2019 (table 1.1). The accuracies of the NAVD 88 elevations were checked by making 56 GPS measurements of established NGS and MassDOT benchmark sites (Massachusetts Department of Transportation, 2018; National Oceanic and Atmospheric Administration, 2018b). The differences between the established NAVD 88 and surveyed GPS elevations for 56 observations ranged from -0.14 to $0.24 \mathrm{ft}$, and the absolute differences had an average of 0.07 $\mathrm{ft}$, a median of $0.06 \mathrm{ft}$, and a root mean squared error of $0.09 \mathrm{ft}$ (table 1.1).

\section{January 2018 Total Water Level Elevations}

Following the January 4 nor'easter, 85 HWMs were flagged along the Massachusetts coastline, but only $80 \mathrm{HWMs}$ were surveyed; the remaining $5 \mathrm{HWMs}$ could not be found or had been destroyed (table 4). Of these 80 HWMs surveyed, 71 HWMs were determined to be accurate, and 9 HWMs were not used because their elevations were substantially lower (at least 2 to $5 \mathrm{ft}$ ) than the elevations at nearby HWMs and peak total water level elevations at the nearby NOAA Boston tide gage. Elevations at these nine HWM outliers appeared to reflect a subsequent lower high total water level elevation based on the NOAA Boston tide gage data (which occurred several hours before the HWMs were flagged), and not the previous peak total water level elevation that occurred on the January 4 storm. Review of field notes and photographs during flagging of these HWMs also aided in determining if the HWMs were not accurate.

The 71 HWMs along the eastern coastline of Massachusetts (U.S. Geological Survey, 2019b; fig. 3) had total water level elevations that ranged from 5.8 to $15.1 \mathrm{ft}$, with an average elevation of $9.4 \mathrm{ft}$ and a median elevation of $9.6 \mathrm{ft}$ (table 5). Fifty percent of the HWMs had elevations between 8.6 to $9.9 \mathrm{ft}$. In four of the five counties, the median HWM elevations were within $1 \mathrm{ft}$ and ranged from 8.9 to $9.8 \mathrm{ft}$. HWM elevations in Barnstable County had a median of $10.9 \mathrm{ft}$, but there were only five sites, which is a limited sample size. The HWM elevations (fig. 3; table 5) show a wide range of variability, but the lowest HWM elevations (fig. 3) were found at sites generally farthest inland from the Atlantic Ocean. These inland sites were not openly exposed to the oncoming direction of winds and waves. The highest HWM elevation values were seen on coastline areas, such as extended beaches, which had direct exposure to contact waves, wave runup, wave setup, and surge from the ocean. These areas may also be open to large areas of great wind fetch off the ocean. In several cases, increased elevations were seen where the surge funneled or piled up into a narrowing bay or estuary where the tides could not quickly drain with the continued winds and consecutive storm surges built on one another.

For coastal areas of eastern New England from Portland to Cape Cod Bay, peak total water level elevations recorded at 10 tide gages and 7 coastal streamgages (fig. 1; table 1) ranged from 4.8 to $11.2 \mathrm{ft}$, with an average of $9.1 \mathrm{ft}$ and a median of $9.6 \mathrm{ft}$ (table 6). These elevations are similar to elevations of nearby HWMs (table 5) and those along this same area of coastline. The tide gages and coastal streamgages also had higher total water level elevations in areas close to the ocean, whereas lower elevations were observed in protected harbors or up tidally affected rivers further from the coast. The southern New England sites (southern coastlines of Connecticut, Rhode Island, and Massachusetts) generally have lower recorded total water level elevations than the eastern and northern New England sites (coastlines from Cape Cod Bay to northern Maine), possibly as a result of land to the north providing a buffer from the northeasterly and easterly winds from the storm. The coastal sites north of Portland generally have higher total water level elevations than sites from Portland south because of the effect of the Bay of Fundy, which has the highest tides in the world (National Oceanic and Atmospheric Administration, 2018c). 
Table 4. High-water marks with no elevation or elevation determined to be inaccurate from the January and March 2018 nor'easters in New England.

[Data are from U.S. Geological Survey (2019b, c). USGS, U.S. Geological Survey; STN, short-term network; no., number; HWM, high-water mark; ID, identifier; ft, foot; NAVD 88, North American Vertical Datum of 1988; - , no data; NOAA, National Oceanic and Atmospheric Administration; MA, Massachusetts; St., Street; Rd., Road]

\begin{tabular}{|c|c|c|c|c|c|c|}
\hline $\begin{array}{l}\text { USGS STN } \\
\text { site no. }\end{array}$ & $\begin{array}{l}\text { USGS } \\
\text { STN } \\
\text { HWM } \\
\text { ID }\end{array}$ & USGS STN site name & $\begin{array}{l}\text { Latitude, } \\
\text { in decimal } \\
\text { degrees }\end{array}$ & $\begin{array}{l}\text { Longitude, } \\
\text { in decimal } \\
\text { degrees }\end{array}$ & $\begin{array}{c}\text { HWM } \\
\text { surveyed } \\
\text { elevation, } \\
\text { in ft above } \\
\text { NAVD } 88\end{array}$ & Reason HWM not used \\
\hline \multicolumn{7}{|c|}{ January 2018 nor'easter } \\
\hline MASUF24069 & 26905 & $\begin{array}{l}\text { Winthrop Ferry Dock and Boat } \\
\text { Launch, Shirley Point, Winthrop, } \\
\text { MA }\end{array}$ & 42.36700 & -70.97100 & - & Not found \\
\hline MASUF24075 & 26915 & $\begin{array}{l}\text { Massachusetts Bay, Winthrop Golf } \\
\text { Club, Winthrop, MA }\end{array}$ & 42.37900 & -70.97570 & - & Destroyed \\
\hline MASUF24089 & 26934 & Long Wharf Pier, Boston, MA & 42.36040 & -71.04790 & 4.4 & Elevation determined to be too low ${ }^{1}$ \\
\hline MASUF24092 & 26937 & $\begin{array}{l}\text { Boston Harbor, Fan Pier, Boston, } \\
\text { MA }\end{array}$ & 42.35350 & -71.04430 & 4.1 & Elevation determined to be too low ${ }^{1}$ \\
\hline MASUF24147 & 27012 & $\begin{array}{l}\text { NOAA tide gage, Fort Point } \\
\text { Channel, Northern Ave., Boston, } \\
\text { MA }\end{array}$ & 42.35480 & -71.05060 & - & Destroyed \\
\hline MASUF04548 & 27026 & $\begin{array}{l}\text { Winthrop Harbor } 707 \text { Shirley St., } \\
\text { Winthrop, MA }\end{array}$ & 42.36849 & -70.97167 & - & Not found \\
\hline MASUF24063 & 28259 & $\begin{array}{l}\text { Boston Harbor, Point Shirley, } \\
\text { Winthrop, MA }\end{array}$ & 42.35780 & -70.97200 & - & $\begin{array}{l}\text { Landowner did not allow it to be } \\
\text { surveyed }\end{array}$ \\
\hline MASUF24092 & 28282 & $\begin{array}{l}\text { Boston Harbor, Fan Pier, Boston, } \\
\text { MA }\end{array}$ & 42.35350 & -71.04430 & 3.7 & Elevation determined to be too low 1 \\
\hline MASUF24089 & 28286 & Long Wharf Pier, Boston, MA & 42.36040 & -71.04790 & 4.1 & Elevation determined to be too low ${ }^{1}$ \\
\hline MASUF24089 & 28287 & Long Wharf Pier, Boston, MA & 42.36040 & -71.04790 & 4.2 & Elevation determined to be too low ${ }^{1}$ \\
\hline MASUF24089 & 28288 & Long Wharf Pier, Boston, MA & 42.36040 & -71.04790 & 4.3 & Elevation determined to be too low ${ }^{1}$ \\
\hline MASUF24089 & 28289 & Long Wharf Pier, Boston, MA & 42.36040 & -71.04790 & 4.2 & Elevation determined to be too low ${ }^{1}$ \\
\hline MAESS04534 & 27027 & 154 Lynnway, Lynn, MA & 42.45964 & -70.94313 & 5.1 & Elevation determined to be too low 2 \\
\hline MANOR04848 & 27023 & $\begin{array}{c}\text { Boston Harbor Transect \#1 near } \\
\text { Bayside Road Quincy MA }\end{array}$ & 42.30121 & -71.00864 & 6.2 & Elevation determined to be too low 3 \\
\hline \multicolumn{7}{|c|}{ March 2018 nor'easter } \\
\hline MABAR04531 & 28217 & $\begin{array}{l}\text { Stage Harbor, Chatham Harbor } \\
\text { Master, Chatham, MA }\end{array}$ & 41.66628 & -69.96644 & 3.3 & Elevation determined to be too low 4 \\
\hline MASUF24670 & 28293 & $\begin{array}{l}\text { Belle Isle Inlet, Read St., Winthrop, } \\
\text { MA }\end{array}$ & 42.38334 & -70.98799 & 13.0 & Elevation determined to be too high 5 \\
\hline MANOR24103 & 28019 & $\begin{array}{l}\text { Weymouth Fore River, } \\
\text { Wessagussett Rd., Weymouth, } \\
\text { MA }\end{array}$ & 42.24970 & -70.94636 & - & Destroyed \\
\hline MASUF24089 & 28290 & Long Wharf Pier, Boston, MA & 42.36040 & -71.04790 & - & Destroyed \\
\hline
\end{tabular}

1Based on a peak of $9.66 \mathrm{ft}$ at the nearby NOAA Boston, Mass., tide gage (8443970) on January 4, 2018, and elevation of $9.3 \mathrm{ft}$ at another nearby HWM (not directly exposed to the ocean). The surveyed elevation approximately coincided with the high-tide elevation of $4.22 \mathrm{ft}$ on January 7, 2018, at about 3 a.m., which was a few hours before this HWM was flagged.

2Based on photograph taken when HWM was flagged, which shows higher ice line in background. Additionally, all other nearby HWMs for the January 2018 nor'easter were about 2 to $3 \mathrm{ft}$ higher.

${ }^{3}$ Based on photograph taken when HWM was flagged and that the March 2018 nor'easter HWM elevation was $9.3 \mathrm{ft}$, substantially higher than the elevation surveyed for the January 2018 nor'easter at the same location. Additionally, all other nearby HWMs for the January 2018 nor'easter were about 3 to $4 \mathrm{ft} \mathrm{higher.}$

4Based on photograph taken on March 5, 2018, when HWM was flagged, showing tide at same elevation. Additionally, all other nearby HWMs for the March 2018 nor'easter were about 4 to $6 \mathrm{ft}$ higher.

5Based on comparison to other HWMs nearby. Additionally, all other nearby HWMs for the March 2018 nor'easter were about 4 to $5 \mathrm{ft}$ lower. 
Table 5. Summary of total water level elevations for high-water marks in coastal areas of Massachusetts following January 2018 nor'easter of and coastal areas of Massachusetts, Connecticut, Rhode Island, New Hampshire, and Maine following the March 2018 nor'easter.

[HWMs, high-water marks; ft, foot; all elevations are at the North American Vertical Datum of 1988; —, not applicable]

\begin{tabular}{|c|c|c|c|c|c|c|c|c|}
\hline \multirow[b]{2}{*}{ State and county } & \multicolumn{2}{|c|}{ High-water marks ${ }^{1}$} & \multicolumn{6}{|c|}{ Elevations, in feet NAVD 88} \\
\hline & $\begin{array}{l}\text { Number } \\
\text { flagged }\end{array}$ & $\begin{array}{l}\text { Number } \\
\text { surveyed }\end{array}$ & Minimum & Maximum & Average & Median & $\begin{array}{c}\text { 25th } \\
\text { percentile }\end{array}$ & $\begin{array}{c}\text { 75th } \\
\text { percentile }\end{array}$ \\
\hline \multicolumn{9}{|l|}{ Massachusetts: } \\
\hline Barnstable County & 5 & 5 & 10.2 & 11.5 & 10.9 & 10.9 & 10.6 & 11.4 \\
\hline Plymouth County & 29 & 29 & 5.8 & 12.6 & 9.1 & 9.4 & 8.4 & 9.8 \\
\hline Suffolk County & 23 & 11 & 6.7 & 9.8 & 8.8 & 8.9 & 8.5 & 9.6 \\
\hline Total & 85 & 71 & 5.8 & 15.1 & 9.4 & 9.6 & 8.6 & 9.9 \\
\hline \multicolumn{9}{|c|}{ March 2-4, 2018, nor'easter } \\
\hline Barnstable County & 15 & 14 & 4.3 & 13.5 & 9.2 & 9.2 & 8.6 & 10.0 \\
\hline Essex County & 9 & 9 & 5.8 & 15.1 & 8.5 & 8.0 & 7.4 & 8.3 \\
\hline Norfolk County & 12 & 11 & 8.3 & 13.5 & 10.1 & 9.7 & 9.2 & 10.6 \\
\hline Plymouth County & 30 & 30 & 6.7 & 14.2 & 9.1 & 9.0 & 8.0 & 9.5 \\
\hline Suffolk County & 20 & 18 & 7.0 & 11.6 & 8.6 & 8.6 & 7.8 & 9.0 \\
\hline Total & 86 & 82 & 4.3 & 15.1 & 8.9 & 9.1 & 8.1 & 9.7 \\
\hline $\begin{array}{l}\text { Portland, Maine, south to Cape Cod, } \\
\text { Massachusetts }{ }^{4}\end{array}$ & 114 & 100 & 5.3 & 15.1 & 8.9 & 8.6 & 7.8 & 9.6 \\
\hline Rhode Island 5 & 2 & 2 & 3.7 & 5.4 & 4.6 & 4.6 & 4.1 & 5.0 \\
\hline
\end{tabular}

IInformation on the location, type, accuracy, and elevation of individual high-water marks is available at U.S. Geological Survey (2019b, c).

2Maine HWM sites are in York County (three sites), Cumberland County (one site), Sagadahoc County (one site), and Waldo County (one site).

${ }^{3}$ New Hampshire HWM sites are all in Rockingham County (13 sites).

4Excludes one site from Barnstable County, which is on the southern side of Cape Cod.

5Rhode Island HWM sites are in Kent County (one site) and Bristol County (one site).

${ }^{6}$ Connecticut HWM sites are in Fairfield County (three sites), New Haven County (two sites), New London County (two sites), and Middlesex County (one site).

${ }^{7}$ Includes one site from Barnstable County, which is on the southern side of Cape Cod. 
Table 6. Summary of total water level elevations at tide gages and coastal streamgages affected by storm tides or tidal backwater during the January and March 2018 nor'easters in New England.

[Station numbers (nos.) and descriptions of U.S. Geological Survey (USGS), National Oceanic and Atmospheric Administration (NOAA), and U.S. Army Corps of Engineers (USACE) tide gages and coastal streamgages affected by storm tides or tidal backwater are listed in table 1 and locations are shown on figure 1. Data for USGS tide gages and coastal streamgages are from U.S. Geological Survey (2019d); for NOAA tide gages, from National Oceanic and Atmospheric Administration (2019e); and for USACE tide gages, from U.S. Army Corps of Engineers (2019). ft, foot; NAVD 88, North American Vertical Datum of 1988; MLLW, mean lower-low water; NGVD 29, National Geodetic Vertical Datum of 1929; Rt., Route; St., Street; Ave., Avenue; CT, Connecticut; MA, Massachusetts; ME, Maine; RI, Rhode Island; - , no data or backwater from tide could not be determined]

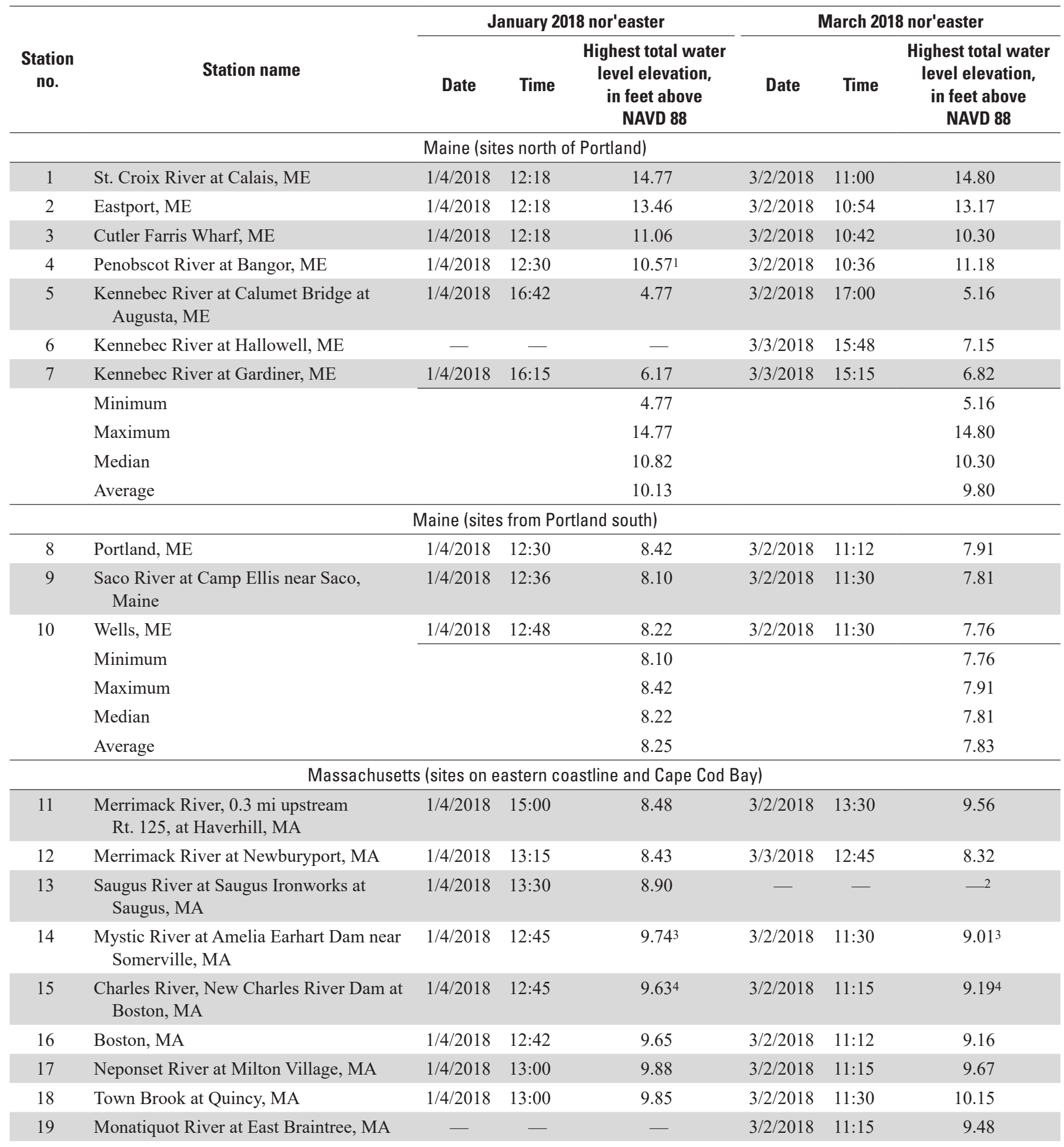


Table 6. Summary of total water level elevations at tide gages and coastal streamgages affected by storm tides or tidal backwater during the January and March 2018 nor'easters in New England._-Continued

[Station numbers (nos.) and descriptions of U.S. Geological Survey (USGS), National Oceanic and Atmospheric Administration (NOAA), and U.S. Army Corps of Engineers (USACE) tide gages and coastal streamgages affected by storm tides or tidal backwater are listed in table 1 and locations are shown on figure 1. Data for USGS tide gages and coastal streamgages are from U.S. Geological Survey (2019d); for NOAA tide gages, from National Oceanic and Atmospheric Administration (2019e); and for USACE tide gages, from U.S. Army Corps of Engineers (2019). ft, foot; NAVD 88, North American Vertical Datum of 1988; MLLW, mean lower-low water; NGVD 29, National Geodetic Vertical Datum of 1929; Rt., Route; St., Street; Ave., Avenue; CT, Connecticut; MA, Massachusetts; ME, Maine; RI, Rhode Island; —, no data or backwater from tide could not be determined]

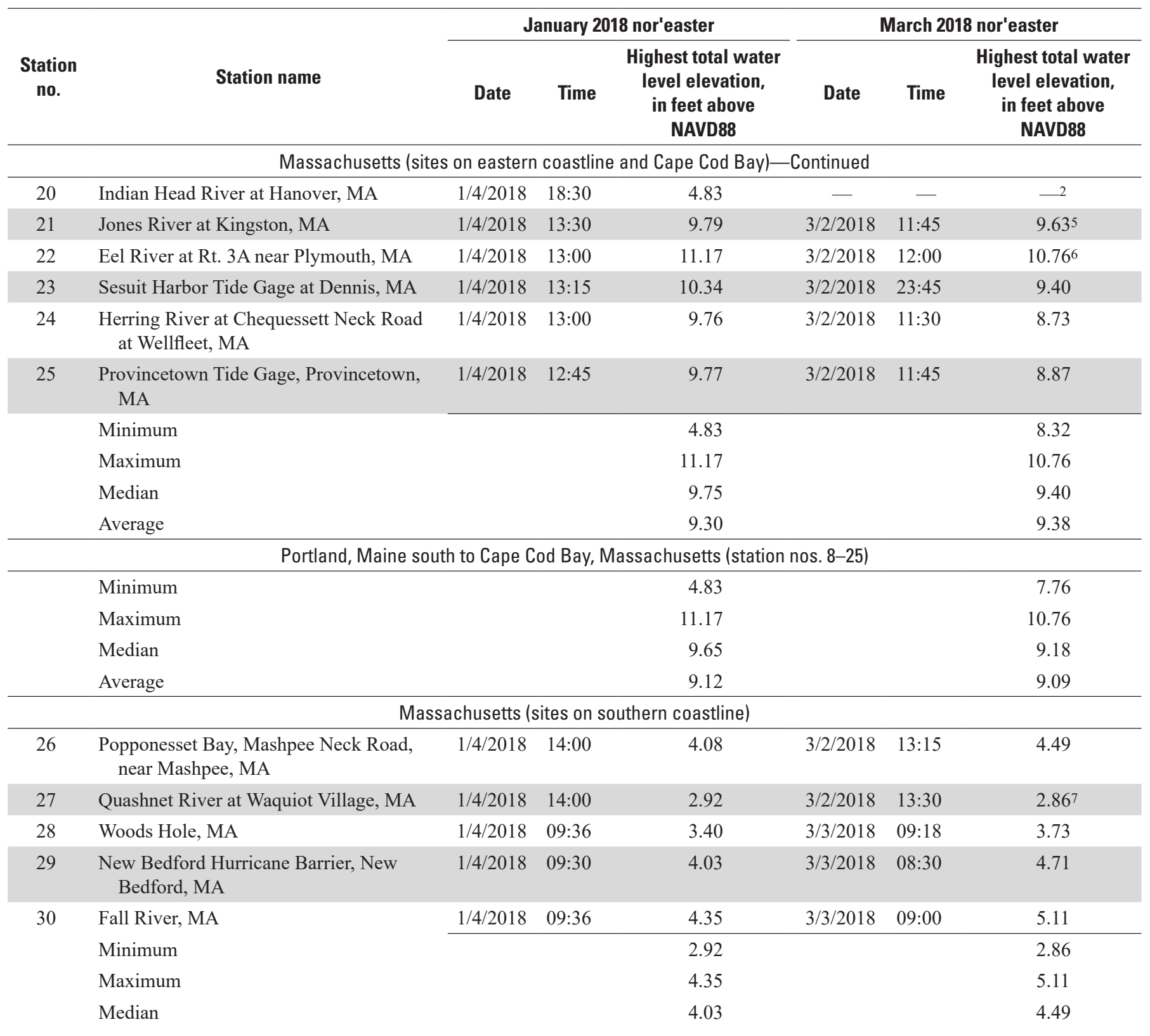


Table 6. Summary of total water level elevations at tide gages and coastal streamgages affected by storm tides or tidal backwater during the January and March 2018 nor'easters in New England.—Continued

[Station numbers (nos.) and descriptions of U.S. Geological Survey (USGS), National Oceanic and Atmospheric Administration (NOAA), and U.S. Army Corps of Engineers (USACE) tide gages and coastal streamgages affected by storm tides or tidal backwater are listed in table 1 and locations are shown on figure 1. Data for USGS tide gages and coastal streamgages are from U.S. Geological Survey (2019d); for NOAA tide gages, from National Oceanic and Atmospheric Administration (2019e); and for USACE tide gages, from U.S. Army Corps of Engineers (2019). ft, foot; NAVD 88, North American Vertical Datum of 1988; MLLW, mean lower-low water; NGVD 29, National Geodetic Vertical Datum of 1929; Rt., Route; St., Street; Ave., Avenue; CT, Connecticut; MA, Massachusetts; ME, Maine; RI, Rhode Island; —, no data or backwater from tide could not be determined]

\begin{tabular}{|c|c|c|c|c|c|c|c|}
\hline \multirow[b]{2}{*}{$\begin{array}{l}\text { Station } \\
\text { no. }\end{array}$} & \multirow[b]{2}{*}{ Station name } & \multicolumn{3}{|c|}{ January 2018 nor'easter } & \multicolumn{3}{|c|}{ March 2018 nor'easter } \\
\hline & & Date & Time & $\begin{array}{l}\text { Highest total water } \\
\text { level elevation, } \\
\text { in feet above } \\
\text { NAVD88 }\end{array}$ & Date & Time & $\begin{array}{l}\text { Highest total water } \\
\text { level elevation, } \\
\text { in feet above } \\
\text { NAVD88 }\end{array}$ \\
\hline & Average & & & 3.76 & & & 4.18 \\
\hline \multicolumn{8}{|c|}{ Rhode Island } \\
\hline 31 & Newport, RI & $1 / 4 / 2018$ & 09:36 & 4.28 & $3 / 3 / 2018$ & 20:48 & 4.01 \\
\hline 32 & Providence, RI & $1 / 4 / 2018$ & 09:42 & 4.21 & $3 / 3 / 2018$ & 21:06 & 5.29 \\
\hline 33 & Pawcatuck River at Westerly, RI & - & - & -2 & $3 / 2 / 2018$ & 09:30 & 4.06 \\
\hline \multirow[t]{5}{*}{34} & Watch Hill Cove Tide Gage Westerly, RI & $1 / 4 / 2018$ & 10:30 & 3.29 & $3 / 3 / 2018$ & $22: 15$ & 3.66 \\
\hline & Minimum & & & 3.29 & & & 3.66 \\
\hline & Maximum & & & 4.28 & & & 5.29 \\
\hline & Median & & & 4.21 & & & 4.04 \\
\hline & Average & & & 3.93 & & & 4.26 \\
\hline \multicolumn{8}{|c|}{ Connecticut } \\
\hline 35 & New London, Thames River, CT & $1 / 4 / 2018$ & 11:06 & 3.31 & $3 / 3 / 2018$ & $22: 42$ & 3.68 \\
\hline 36 & Connecticut River at Essex, CT & $1 / 4 / 2018$ & $12: 30$ & 3.10 & $3 / 4 / 2018$ & 00:25 & 4.41 \\
\hline 37 & Connecticut River at Old Lyme, CT & $1 / 4 / 2018$ & $12: 10$ & 3.50 & $3 / 4 / 2018$ & 00:10 & 4.34 \\
\hline 38 & $\begin{array}{l}\text { Connecticut River near Bridge St. near } \\
\text { Old Saybrook, CT }\end{array}$ & $1 / 4 / 2018$ & 12:00 & 4.04 & $3 / 3 / 2018$ & 23:54 & 4.54 \\
\hline 39 & Bridgeport, CT & $1 / 4 / 2018$ & 12:18 & 5.79 & $3 / 2 / 2018$ & $11: 24$ & 6.45 \\
\hline 40 & Saugatuck River at Rt. 1 at Westport, CT & $1 / 4 / 2018$ & $12: 42$ & 5.92 & $3 / 2 / 2018$ & 11:42 & 6.79 \\
\hline 41 & Stamford Hurricane Barrier, Stamford, CT & $1 / 4 / 2018$ & $12: 30$ & 6.16 & $3 / 2 / 2018$ & $11: 30$ & 6.90 \\
\hline \multirow[t]{5}{*}{42} & $\begin{array}{l}\text { Greenwich Harbor at Grass Island at } \\
\text { Greenwich, CT }\end{array}$ & $1 / 4 / 2018$ & 12:18 & 6.46 & $3 / 2 / 2018$ & $11: 54$ & 6.95 \\
\hline & Minimum & & & 3.10 & & & 3.68 \\
\hline & Maximum & & & 6.46 & & & 6.95 \\
\hline & Median & & & 4.92 & & & 5.50 \\
\hline & Average & & & 4.78 & & & 5.51 \\
\hline
\end{tabular}

1There was a total water level elevation higher on January 3, 2018, but this is not associated with the January 4, 2018, nor'easter.

${ }^{2}$ The total water level is not reported since there is either no tidal backwater (that is, all freshwater runoff) or the total water level is mainly from freshwater runoff.

${ }^{3}$ The total water level reported is for at tide gage on the "ocean side" or "downstream side" of the Amelia Earhart Dam locks. There is also a stage recorded on the "river side" or "upstream side" of the Amelia Earhart Dam locks, but this is not reported since it is the stage of the Mystic River.

4The total water level reported is for at tide gage on the "ocean side" or "downstream side" of the new Charles River Dam locks. There is also a stage recorded on the "river side" or "upstream side" of the new Charles River Dam locks, but this is not reported since it is the stage of the Charles River.

${ }^{5}$ There was a total water level elevation higher on Marth 4, 2018, but this total water level is mainly affected by freshwater runoff.

${ }^{6}$ There was a total water level elevation higher on March 3, 2018, but this total water level is mainly affected by freshwater runoff.

${ }^{7}$ Peak total water level elevation is from crest-stage gage reading because streamgage was not operational. 


\section{March 2018 Total Water Level Elevations}

Following the March 2018 nor'easter, 115 HWMs were flagged along the New England coastline, but only 113 HWMs were recovered; the remaining $2 \mathrm{HWMs}$ had been destroyed (table 4). Of the 113 HWMs surveyed, 111 HWMs (fig. 4; U.S. Geological Survey, 2019c) were determined to be useable, and 2 HWMs (table 4) were not used because their total water level elevations were deemed to be inaccurate. One HWM had an elevation substantially lower (at least 4 to $5 \mathrm{ft}$ ) than elevations at nearby HWMs and tide gages, and the other HWM had an elevation substantially higher (at least 4 to $6 \mathrm{ft}$ ) than elevations at nearby HWMs and tide gages. Review of field notes and photographs during flagging of these HWMs also aided in determining if they were not accurate.

The 82 HWMs primarily along the eastern coastline of Massachusetts (and one location on the southern coastline of Cape Cod; fig. 4A) had total water level elevations that ranged from 4.3 to $15.1 \mathrm{ft}$, with an average elevation of $8.9 \mathrm{ft}$ and a median elevation of $9.1 \mathrm{ft}$ (table 5). Fifty percent of the HWMs had elevations between 8.1 and $9.7 \mathrm{ft}$. In four of the five counties the median HWM elevations were fairly similar, ranging from 8.6 to $9.7 \mathrm{ft}$. The $13 \mathrm{HWMs}$ along the coastline of New Hampshire (fig. $4 B$ ) had elevations ranging from 5.3 to $9.7 \mathrm{ft}$, with an average elevation of $7.6 \mathrm{ft}$ and a median elevation of $7.6 \mathrm{ft}$ (table 5). The $6 \mathrm{HWMs}$ along the coastline of Maine (fig. $4 B$ ) had elevations that ranged from 7.6 to $11.4 \mathrm{ft}$, with an average elevation of $7.8 \mathrm{ft}$ and a median elevation of $8.4 \mathrm{ft}$ (table 5). The average and median HWM elevations of New Hampshire and Maine are fairly similar to those at Essex County (north of Boston) in Massachusetts (within $1.0 \mathrm{ft}$ ), to the south of the New Hampshire and Maine sites. These 100 HWMs along the eastern coastline of New England from Portland to Cape Cod (excluding the one HWM on the southern side of Cape Cod facing Nantucket Sound in Barnstable County) had elevations that ranged from 5.3 to $15.1 \mathrm{ft}$, with an average of $8.9 \mathrm{ft}$ and a median of $8.6 \mathrm{ft}$ (table 5). The remaining 11 HWMs along the southern coastline of New England in Connecticut (8 HWMs; fig. 4C), Rhode Island (2 HWMs; fig. $4 A$ ), and Massachusetts (1 HWM on the southern side of Cape Cod facing Nantucket Sound in Barnstable County; fig. $4 A$ ) had elevations that ranged from 3.1 to $7.5 \mathrm{ft}$, with an average of $4.3 \mathrm{ft}$ and a median of $4.9 \mathrm{ft}$. The HWMs along the easterly or northerly facing coastline of Massachusetts, New Hampshire, and Maine had average and median elevations that were notably higher ( 4.6 and $3.7 \mathrm{ft}$, respectively) than the HWM elevations in Rhode Island and Connecticut along the southern coastline of New England. As was the case for the January 2018 nor'easter, the total water level elevations at the HWMs for the March 2018 nor'easter (fig. 4; table 5) show a wide range, but generally were higher at locations that were directly exposed to waves, wave runup, wave setup, and surge from the ocean. The lower elevations tended to be at locations that were protected from the ocean or further up a tidal river.
For coastal areas of eastern Massachusetts and Cape Cod Bay and from Portland south to the New Hampshire border, peak total water level elevations for the March 2018 nor'easter at 10 tide gages and 6 coastal streamgages (fig. 1; table 1) ranged from 7.8 to $10.8 \mathrm{ft}$, with an average elevation of $9.1 \mathrm{ft}$ and a median elevation of $9.2 \mathrm{ft}$ (table 6). These elevations are similar to elevations of nearby HWMs (table 5). The tide gages and coastal streamgages also had higher total water level elevations in areas close to the ocean, and lower elevations were in protected harbors or up tidally affected rivers further from the coast.

The southern New England sites (southern coastal sites of Massachusetts, Rhode Island, and Connecticut) generally have lower elevations than the eastern coastal sites since the storm winds are from the northeast and east (table 6). The coastal sites north of Portland generally have higher total water level elevations than sites from Portland south because of the effect of the Bay of Fundy, which has the highest tides in the world (National Oceanic and Atmospheric Administration, 2018c).

For the March 2018 nor'easter, 35 USGS temporary water-level sensors were deployed along the Maine, New Hampshire, Massachusetts, and Connecticut coasts (fig. 1; table 2). Of the 35 temporary water-level sensors, 12 were deployed in Connecticut; 14, in Massachusetts; 2, in New Hampshire; and 7, in Maine (table 7). Two sites (one in Massachusetts and one in Maine) malfunctioned and did not record any data. For the 19 temporary storm-tide sensors along the eastern coastline from Portland to Cape Cod Bay (stations 46 to 65 , station 57 recorded no data), the filtered peak total water level elevations ranged from 6.2 to $10.4 \mathrm{ft}$, with an average of $8.5 \mathrm{ft}$ and a median of $8.7 \mathrm{ft}$ (table 7). The peak filtered elevations were also compared with HWMs obtained near the temporary water-level sensors, and averages and medians were similar. The average and median filtered peak elevations at the temporary water-level sensors were similar to all HWMs collected along the coastline (table 5) and to the peak elevations at the tide gages and coastal streamgages (table 6).

\section{Comparison of January and March 2018 Total Water Level Elevations}

A direct comparison of the January and March 2018 nor'easter total water level data along the eastern New England coastline from Portland to Cape Cod Bay was made with data from the tide gages, coastal streamgages, and HWM locations where data exist for both nor'easters. Ten tide gages and five coastal streamgages had recorded peak total water level elevations for both the January and March 2018 nor'easters; the peak total water level elevations ranged from 8.1 to $11.2 \mathrm{ft}$ for the January nor'easter and from 7.8 to $10.8 \mathrm{ft}$ for the March nor'easter (fig. 6). Average elevations for the January and March nor'easters were 9.4 and $9.1 \mathrm{ft}$, respectively, and medians were 9.7 and $9.2 \mathrm{ft}$, respectively. Thus, for the January nor'easter, average and median total water level elevations were about 0.3 to $0.5 \mathrm{ft}$ higher, respectively, than for the March nor'easter. 
Table 7. Summary of total water level elevations at U.S. Geological Survey temporary storm-tide sensors during the March 2-4, 2018, nor'easter in New England.

[Station numbers (nos.) and descriptions of U.S. Geological Survey (USGS) temporary water-level sensors are listed in table 2 and locations are shown on figure 1. HWM, high-water mark; STN, short-term network; NAVD 88, North American Vertical Datum of 1988; ft, foot; ID, identifier; St., Street; Rd., Road; Rt., Route; Ln., Lane; Dr., Drive; CT, Connecticut; MA, Massachusetts; ME, Maine; NH, New Hampshire; —, no data]

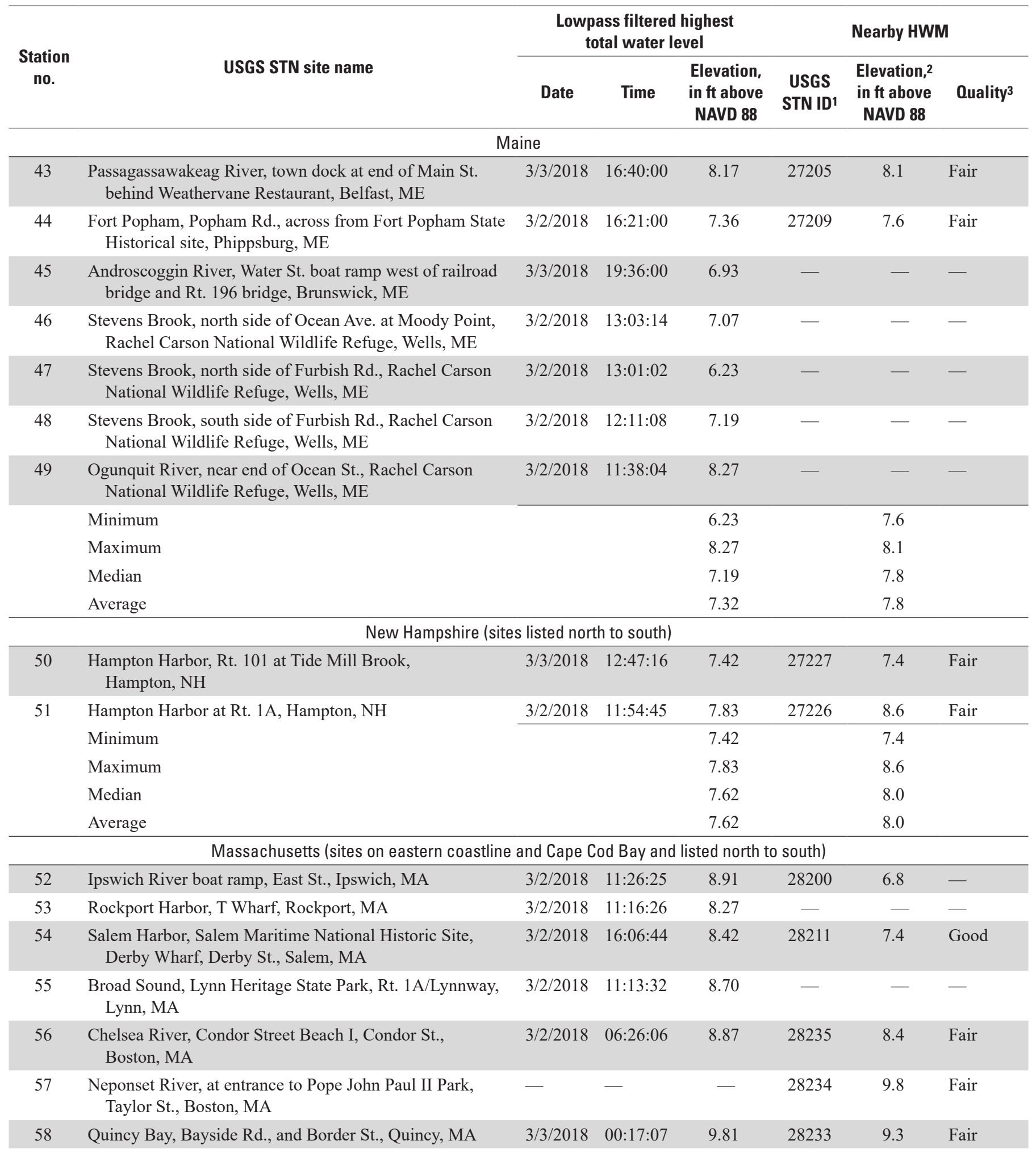


Table 7. Summary of total water level elevations at U.S. Geological Survey temporary storm-tide sensors during the March 2-4, 2018, nor'easter in New England.-Continued

[Station numbers (nos.) and descriptions of U.S. Geological Survey (USGS) temporary water-level sensors are listed in table 2 and locations are shown on figure 1. HWM, high-water mark; STN, short-term network; NAVD 88, North American Vertical Datum of 1988; ft, foot; ID, identifier; St., Street; Rd., Road; Rt., Route; Ln., Lane; Dr., Drive; CT, Connecticut; MA, Massachusetts; ME, Maine; NH, New Hampshire; —, no data]

\begin{tabular}{|c|c|c|c|c|c|c|c|}
\hline \multirow{2}{*}{$\begin{array}{c}\text { Station } \\
\text { no. }\end{array}$} & \multirow[b]{2}{*}{ USGS STN site name } & \multicolumn{3}{|c|}{$\begin{array}{l}\text { Lowpass filtered highest } \\
\text { total water level }\end{array}$} & \multicolumn{3}{|c|}{ Nearby HWM } \\
\hline & & Date & Time & $\begin{array}{c}\text { Elevation, } \\
\text { in } \mathrm{ft} \text { above } \\
\text { NAVD } 88\end{array}$ & $\begin{array}{l}\text { USGS } \\
\text { STN ID1 }\end{array}$ & $\begin{array}{c}\text { Elevation, } \\
\text { in } \mathrm{ft} \text { above } \\
\text { NAVD } 88\end{array}$ & Quality ${ }^{3}$ \\
\hline \multicolumn{8}{|c|}{ Massachusetts (sites on eastern coastline and Cape Cod Bay and listed north to south)_Continued } \\
\hline 59 & $\begin{array}{l}\text { Cohasset Harbor, boat ramp and pier, Lighthouse Ln., } \\
\text { Cohasset, MA }\end{array}$ & $3 / 2 / 2018$ & $16: 25: 33$ & 8.68 & 27718 & 9.1 & Fair \\
\hline 60 & $\begin{array}{l}\text { Scituate Harbor, boat ramp and pier, Jericho Rd., Scituate, } \\
\text { MA }\end{array}$ & $3 / 2 / 2018$ & $11: 32: 24$ & 8.93 & 27720 & 9.3 & Fair \\
\hline 61 & $\begin{array}{l}\text { Green Harbor, boat ramp and dock, Town Pier Rd. and } \\
\text { Central St., Marshfield, MA }\end{array}$ & $3 / 2 / 2018$ & $18: 53: 58$ & 8.78 & 27723 & 8.6 & Fair \\
\hline 62 & Kingston Bay, boat ramp, Brick Kiln Rd., Plymouth, MA & $3 / 2 / 2018$ & $11: 36: 19$ & 9.11 & 28224 & 9.7 & Good \\
\hline 65 & $\begin{array}{l}\text { Rock Harbor, boat ramp and dock, Bayview Dr., Orleans, } \\
\text { MA }\end{array}$ & $3 / 2 / 2018$ & $23: 54: 21$ & 9.34 & 28228 & 8.6 & Excellent \\
\hline & Minimum & & & 8.27 & & 6.8 & \\
\hline & Maximum & & & 10.38 & & 10.1 & \\
\hline & Median & & & 8.91 & & 8.8 & \\
\hline & Average & & & 9.06 & & 8.8 & \\
\hline \multicolumn{8}{|c|}{ Portland, Maine south to Cape Cod Bay, Massachusetts (station nos. 46-65) } \\
\hline & Minimum & & & 6.23 & & 6.8 & \\
\hline 67 & $\begin{array}{l}\text { Poquonuck River Bluff Point, dike at end of Depot Rd., } \\
\text { Groton, CT }\end{array}$ & $3 / 3 / 2018$ & $22: 55: 47$ & 3.34 & 28201 & 3.2 & Good \\
\hline 68 & Niantic Bay, Attawan Beach area, Shore Rd., Niantic, CT & $3 / 3 / 2018$ & 23:07:34 & 3.73 & - & - & - \\
\hline 69 & Hawks Nest Beach, West End Dr., Old Lyme, CT & $3 / 3 / 2018$ & $23: 33: 21$ & 4.21 & - & - & - \\
\hline 70 & $\begin{array}{l}\text { Back River, downstream of Rt. } 154 \text { bridge, Old Saybrook, } \\
\text { CT }\end{array}$ & $3 / 4 / 2018$ & $00: 33: 53$ & 3.98 & 28023 & 4.1 & Good \\
\hline 71 & $\begin{array}{l}\text { Clinton Harbor, Cedar Island Marina, Riverside Dr., } \\
\text { Clinton, CT }\end{array}$ & $3 / 4 / 2018$ & $00: 25: 19$ & 4.94 & - & - & - \\
\hline 72 & $\begin{array}{l}\text { East River, boat launch at end of Old Post Rd., } \\
\text { Madison, CT }\end{array}$ & $3 / 3 / 2018$ & $13: 16: 12$ & 4.87 & 28296 & 3.7 & Good \\
\hline 73 & $\begin{array}{l}\text { West River, downstream side of Rt. } 146 \text { bridge, Guilford, } \\
\text { CT }\end{array}$ & $3 / 3 / 2018$ & $19: 47: 45$ & 5.30 & 28295 & 5.4 & Good \\
\hline 74 & Branford Point Pier, end of Harbor St., Branford, CT & $3 / 2 / 2018$ & $11: 27: 58$ & 5.89 & - & - & - \\
\hline 75 & Cosey Beach, Caroline Rd., East Haven, CT & $3 / 2 / 2018$ & 11:03:59 & 5.96 & - & - & - \\
\hline
\end{tabular}


Table 7. Summary of total water level elevations at U.S. Geological Survey temporary storm-tide sensors during the March 2-4, 2018, nor'easter in New England.-Continued

[Station numbers (nos.) and descriptions of U.S. Geological Survey (USGS) temporary water-level sensors are listed in table 2 and locations are shown on figure 1. HWM, high-water mark; STN, short-term network; NAVD 88, North American Vertical Datum of 1988; ft, foot; ID, identifier; St., Street; Rd., Road; Rt., Route; Ln., Lane; Dr., Drive; CT, Connecticut; MA, Massachusetts; ME, Maine; NH, New Hampshire; —, no data]

\begin{tabular}{|c|c|c|c|c|c|c|c|}
\hline \multirow{2}{*}{$\begin{array}{l}\text { Station } \\
\text { no. }\end{array}$} & \multirow[b]{2}{*}{ USGS STN site name } & \multicolumn{3}{|c|}{$\begin{array}{l}\text { Lowpass filtered highest } \\
\text { total water level }\end{array}$} & \multicolumn{3}{|c|}{ Nearby HWM } \\
\hline & & Date & Time & $\begin{array}{c}\text { Elevation, } \\
\text { in } \mathrm{ft} \text { above } \\
\text { NAVD } 88\end{array}$ & $\begin{array}{l}\text { USGS } \\
\text { STN ID1 }\end{array}$ & $\begin{array}{c}\text { Elevation, }{ }^{2} \\
\text { in } \mathrm{ft} \text { above } \\
\text { NAVD } 88\end{array}$ & Quality ${ }^{3}$ \\
\hline \multicolumn{8}{|c|}{ Connecticut (sites listed east to west) —Continued } \\
\hline 76 & $\begin{array}{l}\text { Norwalk River, Norwalk Maritime Aquarium public dock, } \\
\text { Fairfield, CT }\end{array}$ & $3 / 2 / 2018$ & 11:08:36 & 6.87 & 27700 & 7.0 & Good \\
\hline \multirow[t]{4}{*}{77} & $\begin{array}{l}\text { Public dock on Grass Island Rd., Belle Haven, Greenwich, } \\
\text { CT }\end{array}$ & $3 / 2 / 2018$ & $16: 08: 30$ & 7.08 & 28204 & 7.5 & Fair \\
\hline & Minimum & & & 3.34 & & 3.2 & \\
\hline & Maximum & & & 7.08 & & 7.5 & \\
\hline & Median & & & 4.91 & & 4.7 & \\
\hline
\end{tabular}

IUSGS high-water mark number from U.S. Geological Survey (2019c).

${ }^{2}$ USGS high-water mark elevations are only reported to the tenth of a foot.

${ }_{3}^{3}$ See table 3 for HWM quality rating information.

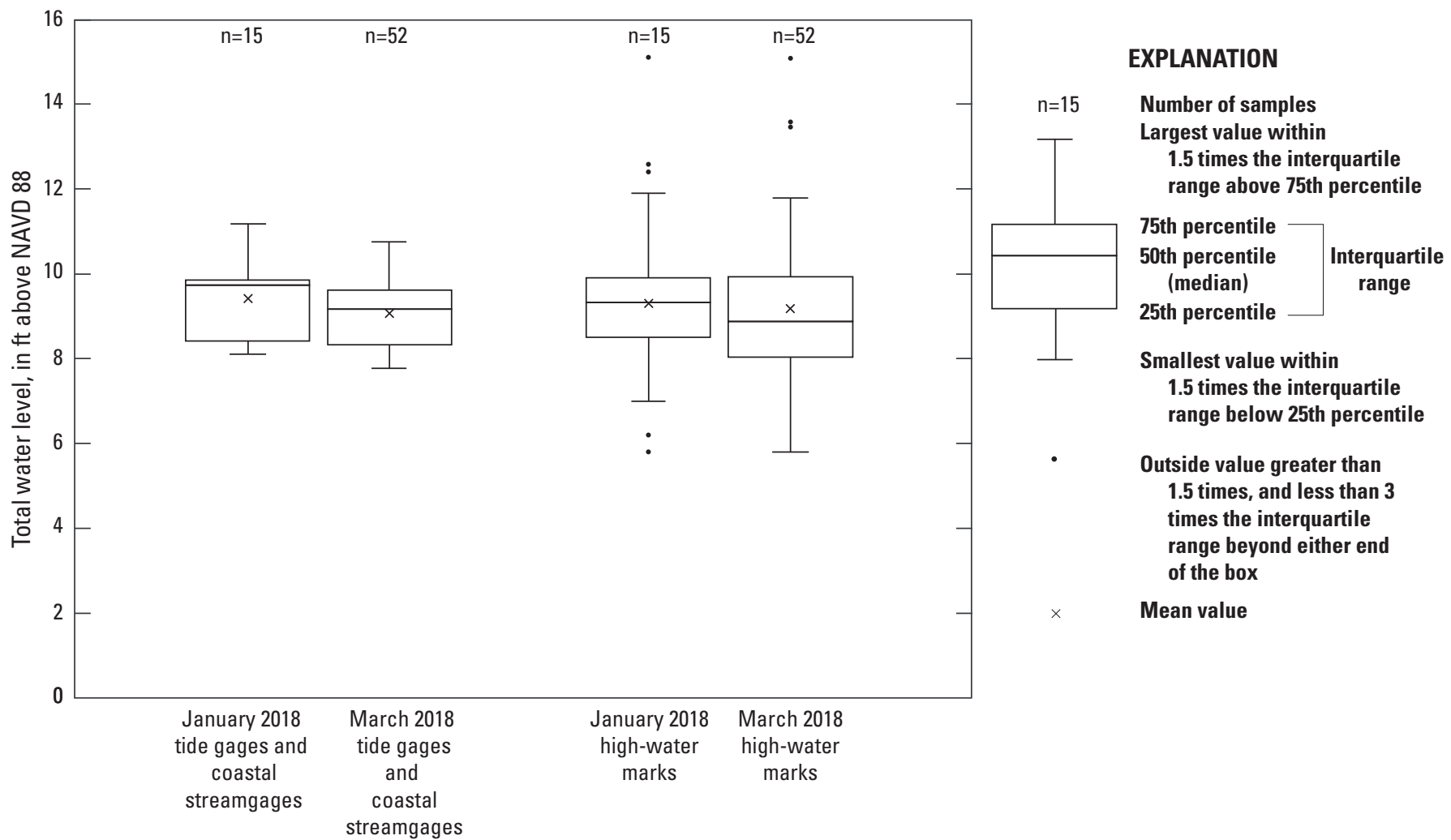

Figure 6. Total water level elevations at 10 tide gages and 5 coastal streamgages affected tidally or by tidal backwater, and 52 high-water mark locations with data for both the January and March 2018 nor'easters from Portland, Maine to Cape Cod Bay, Massachusetts. ft, foot; NAVD 88, North American Vertical Datum of 1988. 
At the 52 HWM locations with data for both the January and March 2018 nor'easters in Massachusetts (the only State with January HWMs), elevations for both events ranged from 5.8 to $15.1 \mathrm{ft}$ (fig. 6). Average HWM elevations for the January and March nor'easters were 9.3 and $9.2 \mathrm{ft}$, respectively, and medians were 9.3 and $8.9 \mathrm{ft}$, respectively. Thus, the January nor'easter had average and median HWM elevations that were 0.1 and $0.4 \mathrm{ft}$ higher, respectively, than the March nor'easter.

\section{Comparison of Observed and Predicted Total Water Level Elevations}

The observed peak total water level data at 26 tide gages along the New England coastline with data for both 2018 nor'easters were compared with the concurrent NOAApredicted total water level data for those locations (table 8). The difference between the observed and predicted total water level elevations for the Boston and Portland NOAA tide gages are shown in fig. $2 A-D$. The difference between the observed and predicted total water level is defined as storm surge (National Oceanic and Atmospheric Administration, 2019g). For this study, only the storm surge at the peak total water level was examined. It is possible that at a lower total water level, the storm surge may have been higher.

For 10 tide gages along the eastern New England coastline from Portland to Cape Cod Bay, the observed peak total water level elevations for the January 4 nor'easter ranged from about 1.6 to $3.7 \mathrm{ft}$ higher than the concurrent predicted elevations, with an average of $2.8 \mathrm{ft}$ and a median of $3.0 \mathrm{ft}$ higher (table 8). For the March 2-4 nor'easter, the observed peak total water level elevation ranged from 1.8 to $4.0 \mathrm{ft}$ higher than the concurrent predicted elevation, with an average of $2.7 \mathrm{ft}$ and a median of $3.0 \mathrm{ft}$ higher. These differences between the observed and predicted peak total water level elevations are approximately the amount of storm surge that was experienced during the highest total water level elevations of the two nor'easters along the coast from Portland to Cape Cod Bay.
A comparison of observed and predicted peak total water level elevations of the January and March 2018 nor'easters at three tide gages north of Portland showed that median differences in observed elevations for the two storms were $1.7 \mathrm{ft}$ and $1.4 \mathrm{ft}$ higher, respectively than the predicted elevations (table 8). For the southern coastline of New England, the median differences of the observed peak total water level elevations at tide gages in Connecticut, Rhode Island, and Massachusetts for the January nor'easter were about 1.6, 1.7, and $1.6 \mathrm{ft}$ higher, respectively, and for the March 2018 nor'easter, $2.6,2.1$, and $2.5 \mathrm{ft}$ higher, respectively, than the corresponding predicted high tide. This is approximately the amount of storm surge that was experienced during the highest total water level elevations of the January and March nor'easters along the coastline north of Portland and the southern coastline of New England.

\section{Uses of Data}

This report documents total water level elevations in the coastal areas of New England from the January and March 2018 nor'easters. The location and elevation of the HWMs, tide gages, coastal streamgages, and temporary water-level sensors used to measure the total water level elevations from the two nor'easter are available from the USGS (https://stn.wim.usgs.gov/fev/), NOAA (https://www.co-ops.nos.noaa.gov/), and USACE (https://reservoircontrol.usace.army.mil/). These data are needed by emergency managers to delineate coastal areas likely to be flooded and to evaluate the extent of such flooding. Federal, State, and local government agencies, nongovernmental organizations, universities, and the public can use these data for land-use planning, flood risk, flood resiliency, and coastal modeling. These entities can use these data to make more informed decisions regarding the management and development of coastal areas shown to be affected by ocean storms within the coastal environment. 
Table 8. Approximate storm surge at 26 tide gages during the January and March 2018 nor'easters in New England.

[Storm surge is the difference between the observed and predicted peak total water level elevation. Station numbers (nos.) and descriptions of U.S. Geological Survey (USGS), National Oceanic and Atmospheric Administration (NOAA), and U.S. Army Corps of Engineers (USACE) tide gages are listed in table 1 and locations are shown on figure 1. Observed total water level elevation USGS tide gages and coastal streamgages are from U.S. Geological Survey (2019d); for NOAA tide gages, from National Oceanic and Atmospheric Administration (2019e); and for USACE tide gages, from U.S. Army Corps of Engineers (2019). Predicted total water level elevation data for USGS and USACE tide gages are from corresponding NOAA tide gages listed in table 1. Predicted total water level elevation data for NOAA tide gages are from the same stations are the observed total water level elevation data. ft, foot; NAVD 88, North American Vertical Datum of 1988; St., Street; MA, Massachusetts; ME, Maine; RI, Rhode Island; CT, Connecticut]

\begin{tabular}{|c|c|c|c|c|c|c|c|}
\hline \multirow[b]{2}{*}{$\begin{array}{l}\text { Station } \\
\text { no. }\end{array}$} & \multirow[b]{2}{*}{$\begin{array}{l}\text { USGS, NOAA, and USACE } \\
\text { station name }\end{array}$} & \multicolumn{3}{|c|}{ January 2018 nor'easter } & \multicolumn{3}{|c|}{ March 2018 nor'easter } \\
\hline & & $\begin{array}{c}\text { Observed } \\
\text { highest total } \\
\text { water level } \\
\text { elevation, } \\
\text { in feet above } \\
\text { NAVD } 88\end{array}$ & $\begin{array}{l}\text { Predicted con- } \\
\text { current total } \\
\text { water level } \\
\text { elevation,1 } \\
\text { in feet above } \\
\text { NAVD } 88\end{array}$ & $\begin{array}{c}\text { Approxi- } \\
\text { mate storm } \\
\text { surge, }{ }^{2} \text { in } \\
\text { feet above } \\
\text { NAVD } 88\end{array}$ & $\begin{array}{c}\text { Observed } \\
\text { highest total } \\
\text { water level } \\
\text { elevation, } \\
\text { in feet above } \\
\text { NAVD } 88\end{array}$ & $\begin{array}{l}\text { Predicted con- } \\
\text { current total } \\
\text { water level } \\
\text { elevation, } 1 \\
\text { in feet above } \\
\text { NAVD } 88\end{array}$ & $\begin{array}{c}\text { Approximate } \\
\text { storm surge, } \\
\text { in feet above } \\
\text { NAVD } 88\end{array}$ \\
\hline \multicolumn{8}{|c|}{ Maine (sites north of Portland) } \\
\hline 1 & St. Croix River at Calais, ME & 14.77 & 12.78 & 1.99 & 14.80 & 12.22 & 2.58 \\
\hline 2 & Eastport, ME & 13.46 & 12.37 & 1.09 & 13.17 & 11.89 & 1.28 \\
\hline \multirow[t]{5}{*}{3} & Cutler Farris Wharf, ME & 11.06 & 9.33 & 1.73 & 10.30 & 8.90 & 1.40 \\
\hline & Minimum & & & 1.09 & & & 1.28 \\
\hline & Maximum & & & 1.99 & & & 2.58 \\
\hline & Median & & & 1.73 & & & 1.40 \\
\hline & Average & & & 1.60 & & & 1.75 \\
\hline \multicolumn{8}{|c|}{ Maine (sites from Portland south) } \\
\hline 8 & Portland, ME & 8.42 & 6.36 & 2.06 & 7.91 & 5.95 & 1.96 \\
\hline 9 & $\begin{array}{l}\text { Saco River at Camp Ellis near } \\
\text { Saco, Maine }\end{array}$ & 8.10 & 6.47 & 1.63 & 7.81 & 5.96 & 1.85 \\
\hline \multirow[t]{5}{*}{10} & Wells, ME & 8.22 & 6.24 & 1.98 & 7.76 & 5.86 & 1.90 \\
\hline & Minimum & & & 1.63 & & & 1.85 \\
\hline & Maximum & & & 2.06 & & & 1.96 \\
\hline & Median & & & 1.98 & & & 1.90 \\
\hline & Average & & & 1.89 & & & 1.90 \\
\hline \multicolumn{8}{|c|}{ Massachusetts (sites on eastern coastline and Cape Cod Bay) } \\
\hline 12 & $\begin{array}{l}\text { Merrimack River at } \\
\text { Newburyport, MA }\end{array}$ & 8.43 & 5.91 & 2.52 & 8.32 & 5.20 & 3.12 \\
\hline 14 & $\begin{array}{l}\text { Mystic River at Amelia Earhart } \\
\text { Dam near Somerville, MA }\end{array}$ & 9.74 & 6.54 & 3.20 & 9.01 & 5.98 & 3.03 \\
\hline 15 & $\begin{array}{l}\text { Charles River, New Charles } \\
\text { River Dam at Boston, MA }\end{array}$ & 9.63 & 6.55 & 3.08 & 9.19 & 6.14 & 3.05 \\
\hline 16 & Boston, MA & 9.65 & 6.56 & 3.09 & 9.16 & 6.15 & 3.01 \\
\hline 23 & $\begin{array}{l}\text { Sesuit Harbor Tide Gage at } \\
\text { Dennis, MA }\end{array}$ & 10.34 & 6.67 & 3.67 & 9.40 & 5.45 & 3.95 \\
\hline 24 & $\begin{array}{l}\text { Herring River at Chequessett } \\
\text { Neck Road at Wellfleet, MA }\end{array}$ & 9.76 & 6.88 & 2.88 & 8.73 & 6.44 & 2.29 \\
\hline 25 & $\begin{array}{l}\text { Provincetown Tide Gage, } \\
\text { Provincetown, MA }\end{array}$ & 9.77 & 6.10 & 3.67 & 8.87 & 5.73 & 3.14 \\
\hline & Minimum & & & 2.52 & & & 2.29 \\
\hline & Maximum & & & 3.67 & & & 3.95 \\
\hline & Median & & & 3.09 & & & 3.05 \\
\hline & Average & & & 3.16 & & & 3.08 \\
\hline
\end{tabular}


Table 8. Approximate storm surge at 26 tide gages during the January and March 2018 nor'easters in New England.-Continued

[Storm surge is the difference between the observed and predicted peak total water level elevation. Station numbers (nos.) and descriptions of U.S. Geological Survey (USGS), National Oceanic and Atmospheric Administration (NOAA), and U.S. Army Corps of Engineers (USACE) tide gages are listed in table 1 and locations are shown on figure 1. Observed total water level elevation USGS tide gages and coastal streamgages are from U.S. Geological Survey (2019d); for NOAA tide gages, from National Oceanic and Atmospheric Administration (2019e); and for USACE tide gages, from U.S. Army Corps of Engineers (2019). Predicted total water level elevation data for USGS and USACE tide gages are from corresponding NOAA tide gages listed in table 1. Predicted total water level elevation data for NOAA tide gages are from the same stations are the observed total water level elevation data. ft, foot; NAVD 88, North American Vertical Datum of 1988; St., Street; MA, Massachusetts; ME, Maine; RI, Rhode Island; CT, Connecticut]

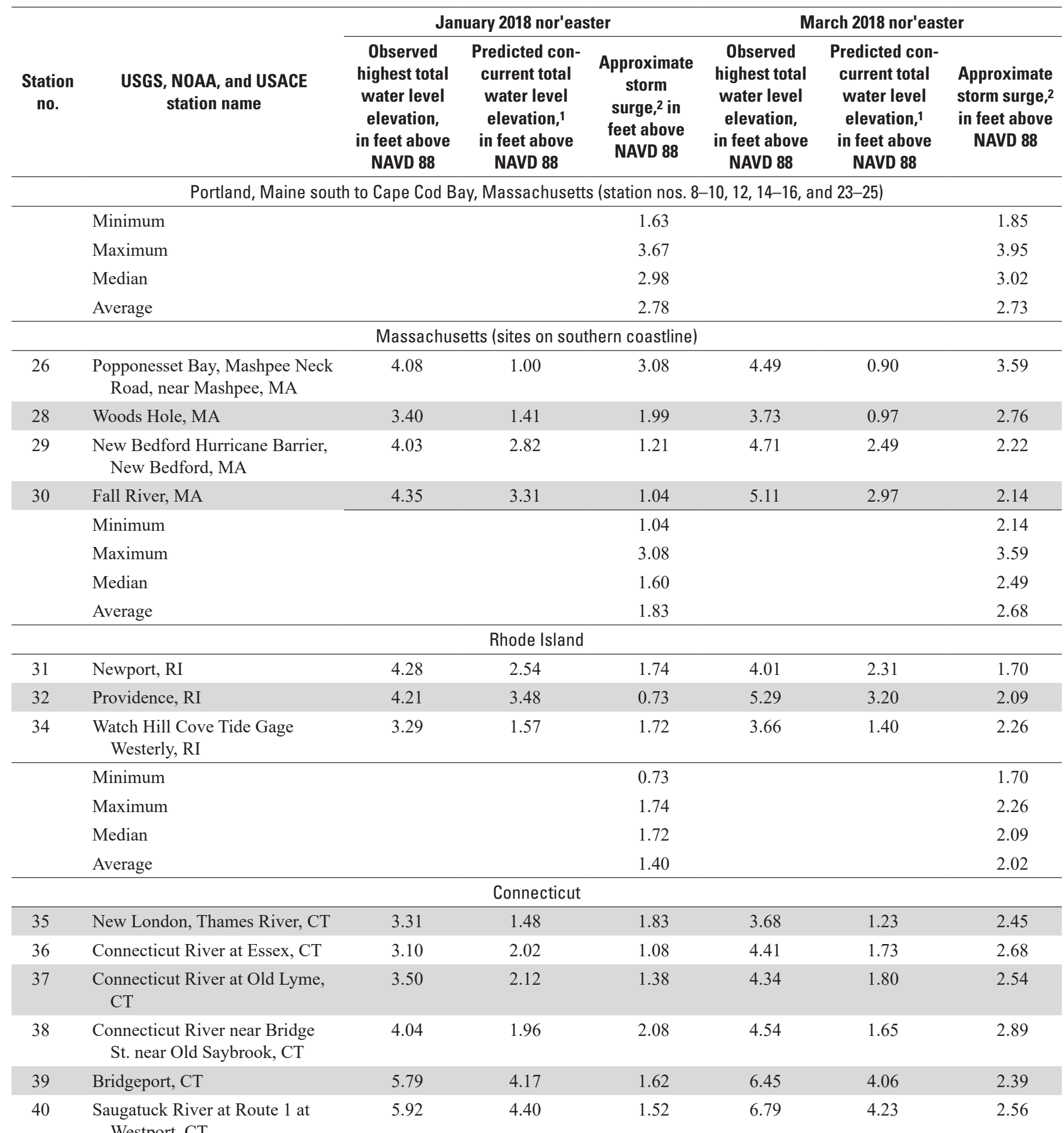


Table 8. Approximate storm surge at 26 tide gages during the January and March 2018 nor'easters in New England.—Continued

[Storm surge is the difference between the observed and predicted peak total water level elevation. Station numbers (nos.) and descriptions of U.S. Geological Survey (USGS), National Oceanic and Atmospheric Administration (NOAA), and U.S. Army Corps of Engineers (USACE) tide gages are listed in table 1 and locations are shown on figure 1. Observed total water level elevation USGS tide gages and coastal streamgages are from U.S. Geological Survey (2019d); for NOAA tide gages, from National Oceanic and Atmospheric Administration (2019e); and for USACE tide gages, from U.S. Army Corps of Engineers (2019). Predicted total water level elevation data for USGS and USACE tide gages are from corresponding NOAA tide gages listed in table 1. Predicted total water level elevation data for NOAA tide gages are from the same stations are the observed total water level elevation data. ft, foot; NAVD 88, North American Vertical Datum of 1988; St., Street; MA, Massachusetts; ME, Maine; RI, Rhode Island; CT, Connecticut]

\begin{tabular}{|c|c|c|c|c|c|c|c|}
\hline \multirow[b]{2}{*}{$\begin{array}{l}\text { Station } \\
\text { no. }\end{array}$} & \multirow[b]{2}{*}{$\begin{array}{l}\text { USGS, NOAA, and USACE } \\
\text { station name }\end{array}$} & \multicolumn{3}{|c|}{ January 2018 nor'easter } & \multicolumn{3}{|c|}{ March 2018 nor'easter } \\
\hline & & $\begin{array}{c}\text { Observed } \\
\text { highest total } \\
\text { water level } \\
\text { elevation, } \\
\text { in feet above } \\
\text { NAVD } 88\end{array}$ & $\begin{array}{l}\text { Predicted con- } \\
\text { current total } \\
\text { water level } \\
\text { elevation, } 1 \\
\text { in feet above } \\
\text { NAVD } 88\end{array}$ & $\begin{array}{c}\text { Approximate } \\
\text { storm } \\
\text { surge, }{ }^{2} \text { in } \\
\text { feet above } \\
\text { NAVD } 88\end{array}$ & $\begin{array}{c}\text { Observed } \\
\text { highest total } \\
\text { water level } \\
\text { elevation, } \\
\text { in feet above } \\
\text { NAVD } 88\end{array}$ & $\begin{array}{l}\text { Predicted con- } \\
\text { current total } \\
\text { water level } \\
\text { elevation, } 1 \\
\text { in feet above } \\
\text { NAVD } 88\end{array}$ & $\begin{array}{c}\text { Approximate } \\
\text { storm surge, }{ }^{2} \\
\text { in feet above } \\
\text { NAVD } 88\end{array}$ \\
\hline \multirow[t]{2}{*}{42} & $\begin{array}{l}\text { Greenwich Harbor at Grass } \\
\text { Island at Greenwich, CT }\end{array}$ & 6.46 & 4.48 & 1.98 & 6.95 & 4.28 & 2.67 \\
\hline & Minimum & & & 1.08 & & & 2.39 \\
\hline
\end{tabular}

${ }^{1}$ The predicted peak total water level elevations at NOAA stations are available from National Oceanic and Atmospheric Administration (2019f) at the mean lower low water datum. NOAA provides conversion of mean lower low water datum elevation to the North American Datum of 1988 or the conversion was done using the NOAA online VDatum program (National Oceanic and Atmospheric Administration, 2019f).

${ }^{2}$ Difference between observed highest and predicted total water level elevations.

3The total water level reported is for a tide gage on the "ocean side" or "downstream side" of the Amelia Earhart Dam locks. There is also a stage recorded on the "river side" or "upstream side" of the Amelia Earhart Dam locks, but this is not reported since it is the stage of the Mystic River.

${ }^{4}$ The total water level reported is for a tide gage on the "ocean side" or "downstream side" of the new Charles River Dam locks. There is also a stage recorded on the "river side" or "upstream side" of the new Charles River Dam locks, but this is not reported since it is the stage of the Charles River. 


\section{Summary}

During January and March 2018, the eastern coastline of New England was impacted by two nor'easters. The U.S. Geological Survey (USGS), under an interagency agreement with the Federal Emergency Management Agency (FEMA), collected total water level data (high-water marks and continuous water-level sensors) in those coastal areas. All total water level data were collected and reported using the North American Vertical Datum of 1988. The total water level is the combination of tide, storm surge, waves (wave runup or wave setup), and freshwater input.

The January 4, 2018, nor'easter formed as an extratropical cyclone off the coast of Florida and the Bahamas on January 3 and quickly moved northward along the east coast of the United States and intensified rapidly. The powerful storm reached the coast of New England on January 4, bringing high winds, heavy snowfall, and coastal flooding to the region. This storm also occurred during astronomical high tides from a full moon on January 1. The National Oceanic and Atmospheric Administration (NOAA) tide gage in Boston, Massachusetts, recorded the highest total water level elevation on record at $9.66 \mathrm{ft}$ on January 4. This elevation exceeded the previous peak elevation of record of $9.52 \mathrm{ft}$ from the February 7, 1978, nor'easter. Flood and wind damages affected many coastal communities of Massachusetts on the North Shore area between Boston and New Hampshire, at Boston Harbor, on the South Coast area between Boston and Cape Cod, along Cape Cod Bay, and on Nantucket. The coastal flooding resulted in numerous rescues and flooded homes and vehicles in Massachusetts.

On March 2-4, a strong nor'easter passed off the coast of New England. This nor'easter started as a stationary front over the Midwest on March 1 and moved eastward off the mid-Atlantic coast, resulting in a new low-pressure system that formed and intensified rapidly. The storm reached the New England coast on March 2, bringing high winds, rainfall and snowfall, and coastal flooding to the region. This storm also occurred during astronomical high tides from a full moon on March 1. The NOAA Boston tide gage recorded a peak total water level elevation of $9.16 \mathrm{ft}$ on March 2, the third highest elevation on record. Many coastal communities from Portland, Maine, southward to Cape Cod Bay experienced severe flooding, resulting in numerous rescues and flooded homes and vehicles. Major disaster declarations were made in many coastal counties of Massachusetts, New Hampshire, and Maine.

Following the January 2018 nor'easter, the USGS identified and flagged 85 high-water marks (HWMs) along coastal areas of eastern Massachusetts from the State border with New Hampshire to Cape Cod Bay. No USGS waterlevel sensors were deployed for the January nor'easter. In preparation for the approach of the March nor'easter, the USGS deployed 35 temporary water-level sensors to collect total water level elevation data before, during, and after the storm along the coastal areas of New England from Portland to the Connecticut-New York State border. Following the March nor'easter, 115 HWMs were identified and flagged in the coastal areas of New England from Portland to the Connecticut-New York State border. Peak total water level elevations were available from 28 tide gages and 14 coastal streamgages affected tidally or by tidal backwater in New England for both the January and March nor'easters.

Following the January and March 2018 nor'easters, field crews identified and flagged HWMs, which included completing a HWM field form with a detail description and sketch of the location, latitude and longitude, a description of the type, distance above ground surface or other object, qualitative description of accuracy, and type of marker, and also photographing the HWM and pathways to it. These HWMs were surveyed from August through November 2018 and in February 2019, except HWMs that were identified and flagged near USGS temporary water-level sensors, tide gages, and coastal streamgages in March, which were surveyed then. Elevations of all HWMs were referenced to the North American Vertical Datum of 1988 (NAVD 88), and horizontal coordinates, to the North American Datum of 1983, using the Global Navigation Satellite System, survey-grade Global Positioning System (GPS) receivers, and automatic level and total station surveying equipment. The GPS elevations were checked for quality assurance against 56 National Geodetic Survey and Massachusetts Department of Transportation benchmarks with established NAVD 88 elevations at locations along the coast and near the HWMs and storm-tide sensors. For the 56 quality-assurance observations, the absolute differences between the GPS elevations and established NAVD 88 elevations had an average of 0.07 foot (ft), a median of $0.06 \mathrm{ft}$, and a root mean square error of $0.09 \mathrm{ft}$.

Of the 85 HWMs flagged for the January nor'easter, only 80 HWMs were surveyed; the remaining 5 could not be found or had been destroyed when locations were revisited to survey. Of these $80 \mathrm{HWMs}$ surveyed, $71 \mathrm{HWM}$ elevations were determined to be accurate, and 9 HWM elevations were determined to be inaccurate based on nearby total water level elevations. The HWMs were determined to have been flagged at a subsequent lower high total water level elevation than the previous peak elevation that occurred on January 4, 2018. Of the 71 HWMs, Massachusetts had total water level elevations that ranged from 5.8 to $15.1 \mathrm{ft}$, with an average of $9.4 \mathrm{ft}$ and a median of $9.6 \mathrm{ft}$. Peak total water level elevations at 10 tide and 7 coastal streamgages from Portland to Cape Cod Bay ranged from 4.8 to $11.2 \mathrm{ft}$, with an average of $9.1 \mathrm{ft}$ and a median of $9.6 \mathrm{ft}$.

Of the 115 HWMs flagged for the March nor'easter, only 113 HWMs were surveyed; the remaining 2 could not be found or had been destroyed when locations were revisited to survey. Of these 113 HWMs surveyed, 111 HWM elevations were determined to be accurate, and 2 elevations were determined to be inaccurate based on the total water level elevations of nearby HWMs or tide gages. Of the 111 HWMs along the eastern New England coastline from Portland to Cape Cod Bay, 100 had total water level elevations that ranged from 
5.3 to $15.1 \mathrm{ft}$, with an average of $8.9 \mathrm{ft}$ and a median of $8.5 \mathrm{ft}$. The remaining 11 HWMs along the southern coastline of New England in Connecticut, Rhode Island, and Massachusetts had elevations that ranged from 3.1 to $7.5 \mathrm{ft}$, with an average of $4.3 \mathrm{ft}$ and a median of $4.9 \mathrm{ft}$. Peak total water level elevations for 19 USGS water-level sensors from Portland to Cape Cod Bay ranged from 6.2 to $10.4 \mathrm{ft}$, with an average of $8.5 \mathrm{ft}$ and a median of $8.7 \mathrm{ft}$. Six storm-tide sensors in Connecticut had peak elevations ranging from 3.3 to $7.1 \mathrm{ft}$, with an average of $5.0 \mathrm{ft}$ and a median of $4.9 \mathrm{ft}$. Peak total water level elevations at 10 tide and 6 coastal streamgages from Portland to Cape Cod Bay ranged from 7.8 to $10.8 \mathrm{ft}$ with an average of $9.1 \mathrm{ft}$ and a median of $9.2 \mathrm{ft}$.

The January and March 2018 nor'easter total water level data along the eastern New England coastline from Portland, Maine, to Cape Cod Bay, Massachusetts, were compared with data from tide gages, coastal streamgages, and HWM locations where data exist for both nor'easters. For the 10 tide gages and 5 coastal streamgages with total water level elevations for both storm events, the average and median elevations for the January nor'easter were about 0.3 and $0.5 \mathrm{ft}$ higher, respectively, than for the March nor'easter. At the $52 \mathrm{HWM}$ locations with total water level elevations for both events (all locations were in Massachusetts as it was the only State with January HWM data) the average and median elevations from the January storm were 0.1 and $0.4 \mathrm{ft}$ higher, respectively than the March nor'easter.

The observed peak total water level elevations at 10 tide gages along the eastern New England coastline from Portland, Maine, to Cape Cod Bay, Massachusetts, with data for both 2018 nor'easters were compared with the concurrent predicted total water level elevations at those locations. For the January nor'easter, the observed peak elevations ranged from 1.6 to $3.7 \mathrm{ft}$ higher than the concurrent predicted elevations, with an average of $2.8 \mathrm{ft}$ and a median of $3.0 \mathrm{ft}$ higher. For the March nor'easter, the observed peak total water level elevations ranged from 1.8 to $4.0 \mathrm{ft}$ higher than the concurrent predicted elevations, with an average of $2.7 \mathrm{ft}$ and a median of $3.0 \mathrm{ft}$ higher. The difference between the observed peak and the concurrent predicted total water level elevations is approximately the amount of storm surge that was experienced during the highest tides of the two nor'easters along the coastline from Portland to Cape Cod Bay.

Total water level data collected following the January and March 2018 nor'easters can be used by Federal, State, and local government agencies, nongovernmental organizations, universities, and the public in understanding the areal extent and depth of the coastal flooding. Additionally, these data can be used for land-use planning, flood risk studies, flood resiliency studies, and coastal models. Total water level data from these nor'easters can be compared with data from past and future regional nor'easters, hurricanes, and tropical storms to assist in future planning and preparedness.

\section{References Cited}

Benson, M.A., and Dalrymple, T., 1967, General field and office procedures for indirect measurements: U.S. Geological Survey Techniques of Water-Resources Investigations, book 3, chap. Al, 30 p., accessed July 22, 2015, at https://doi.org/10.3133/twri03A1.

Caron, C., 2018, Eight dead, including two children, in fierce nor'easter: The New York Times web page, March 3, accessed May 14, 2019, at https://www.nytimes.com/2018/ 03/03/us/noreaster-storm-deaths.html.

Di Liberto, T., 2018, Nor'easters pummel the U.S. northeast in late winter 2018: National Oceanic and Atmospheric Administration ClimateWatch Magazine, March 14, accessed May 10, 2019, at https://www.climate.gov/newsfeatures/event-tracker/nor $\%$ E2\%80\%99easters-pummel-usnortheast-late-winter-2018.

Erdman, J., 2018, Winter storm Grayson's bombogenesis brought whiteout conditions from Virginia to New England, record coastal flooding at Boston Harbor: The Weather Channel, January 5, accessed April 25, 2019, at https://weather.com/storms/winter/news/2018-01-04-winterstorm-grayson-northeast-bombogenesis-snow-forecast.

Feaster, T.D., and Koenig, T.A., 2017, Field manual for identifying and preserving high-water mark data: U.S. Geological Survey Open-File Report 2017-1105, 67 p., accessed June 23, 2019, at https://doi.org/10.3133/ofr20171105.

Federal Emergency Management Agency, 2016, Guidance for flood risk analysis and mappingCoastal notation, acronyms, and glossary of terms: Federal Emergency Management Agency Guidance Document 66, 43 p., accessed October 8, 2019, at https://www.fema.gov/media-library-data/14720018837686c9f486e6449f2686b572b7315d6941d/Coastal_Notation_ Acronyms_Glossary_May_2016.pdf.

Federal Emergency Management Agency, 2018a, Maine severe winter storm and flooding (DR-4367): Federal Emergency Management Agency web page, accessed April 25, 2019, at https://www.fema.gov/disaster/4367.

Federal Emergency Management Agency, 2018b, Massachusetts severe winter storm and flooding (DR-4372): Federal Emergency Management Agency web page, accessed April 25, 2019, at https://www.fema.gov/ disaster/4372.

Federal Emergency Management Agency, 2018c, New Hampshire severe winter storm and flooding (DR-4367): Federal Emergency Management Agency web page, accessed April 25, 2019, at https://www.fema.gov/ disaster/4370. 
Gadoury, R.A., 1979, Coastal flood of February 7, 1978, in Maine, Massachusetts, and New Hampshire: U.S. Geological Water-Resources Investigations 79-61, 57 p., 2 pls., accessed February 22, 2019, at https://doi.org/ 10.3133/wri7961.

Kedzierski, J., 1992, High water marks of the Halloween coastal storm, October 1991: U.S. Army Corps of Engineers, 445 p., accessed February 22, 2019, at https://apps.dtic.mil/dtic/tr/fulltext/u2/a336589.pdf.

Kenney, T.A., 2010, Levels at gaging stations: U.S. Geological Survey Techniques and Methods book 3, chap. A19, 60 p., accessed April 22, 2019, at https://doi.org/10.3133/tm3A19.

KeyNetGPS, Inc., 2018, A Trimble VRS network: KeyNetGPS, Inc. website, accessed August 21, 2018, at http://vrs.keynetgps.com/Map/SensorMap.aspx.

Koenig, T.A., Bruce, J.L., O’Connor, J.E., McGee, B.D., Holmes, R.R., Jr., Hollins, R., Forbes, B.T., Kohn, M.S., Schellekens, M.F., Martin, Z.W., and Peppler, M.C., 2016, Identifying and preserving high-water mark data: U.S. Geological Survey Techniques and Methods, book 3, chap. A24, 47 p., accessed August 23, 2019, at https://doi.org/10.3133/tm3A24.

Massachusetts Department of Transportation, 2018, Geodetic control: Massachusetts Department of Transportation map viewer, accessed August 21, 2018, at https://gis.massdot.state.ma.us/geodeticcontrol/.

Massey, A.J., and Verdi, R.J., 2015, Storm tide monitoring during the blizzard of January 26-28, 2015, in eastern Massachusetts: U.S. Geological Survey Open-File Report 2015-1081, 8 p., accessed May 2, 2019, at https://doi.org/ $10.3133 /$ ofr20151081.

National Oceanic and Atmospheric Administration, 2018a, Top ten highest water levels for long-term stations in feet above MHHW (as of 4/2018): National Oceanic and Atmospheric Administration, 7 p., accessed April 25, 2019, at https://www.CO-OPS.nos.noaa.gov/est/Top10 form_ft.pdf.

National Oceanic and Atmospheric Administration, 2018b, Survey marks and datasheets: National Oceanic and Atmospheric Administration web page, accessed August 21, 2018, at https://www.ngs.noaa.gov/datasheets/index.shtml.

National Oceanic and Atmospheric Administration, 2018c, Where is the highest tide?: National Oceanic and Atmospheric Administration web page, August 13, accessed April 22, 2019, at https:/oceanservice.noaa.gov/facts/ highesttide.html.

National Oceanic and Atmospheric Administration, 2019a, Introduction to storm surge: National Oceanic and Atmospheric Administration National Hurricane Center web page, accessed September 19, 2019, at https://www.nhc.noaa.gov/surge/surge_intro.pdf.
National Oceanic and Atmospheric Administration, 2019b, 8443970 Boston, MA: National Oceanic and Atmospheric Administration Tides \& Currents web page, accessed September 20, 2019, at https://tidesandcurrents.noaa.gov/ waterlevels.html?id=8443970.

National Oceanic and Atmospheric Administration, 2019c, 8418150 Portland, ME: National Oceanic and Atmospheric Administration Tides \& Currents web page, accessed September 20, 2019, at https://tidesandcurrents.noaa.gov/ waterlevels.html?id=8418150.

National Oceanic and Atmospheric Administration, 2019d, Shoreline mileage of the United States: National Oceanic and Atmospheric Administration web page, accessed February 19, 2019, at https://coast.noaa.gov/data/docs/ states/shorelines.pdf.

National Oceanic and Atmospheric Administration, 2019e, Water levels — Station selection: National Oceanic and Atmospheric Administration Tides \& Current website, accessed September 19, 2019, at https://tidesandcurrents.noaa.gov/stations.html?type= Water+Levels.

National Oceanic and Atmospheric Administration, 2019f, Online vertical datum transformation: National Oceanic and Atmospheric Administration website, accessed May 17, 2019, at https://vdatum.noaa.gov/welcome.html.

National Oceanic and Atmospheric Administration, 2019g, What is storm surge?: National Oceanic and Atmospheric Administration web page, accessed May 17, 2019, at https://oceanservice.noaa.gov/facts/stormsurgestormtide.html.

National Oceanic and Atmospheric Administration, 2020, Orthometric height conversion: National Oceanic and Atmospheric Administration National Geodetic Survey website, accessed February 18, 2020, at https://www.ngs.noaa.gov/cgi-bin/VERTCON/vert_con.prl.

National Weather Service, 2018a, Eastern U.S. winter storm: National Oceanic and Atmospheric Administration Weather Prediction Center website, accessed May 8, 2019, at https://www.wpc.ncep.noaa.gov/storm_summaries/2018/ storm1/storm1_archive.shtml.

National Weather Service, 2018b, Great Lakes to New England winter storm with coastal wind and rain: National Oceanic and Atmospheric Administration Weather Prediction Center website, accessed May 10, 2019, at https://www.wpc.ncep.noaa.gov/storm_summaries/2018/ storm10/storm10_archive.shtml.

National Weather Service, 2018c, January 4, 2018 blizzard: National Oceanic and Atmospheric Administration National Weather Service web page, accessed May 8, 2019, at https:/www.weather.gov/okx/Blizzard_Jan42018. 
New England Cable News, 2018, Major flooding wreaks havoc along New England coast: New England Cable News, January 4, accessed April 8, 2019, at https://www.necn.com/ news/national-international/significant-flooding-a-cause-forconcern-along-massachusetts-coast/2019859/.

Ostiguy, L.J., Sargent, T.C., Izbicki, B.J., and Bent, G.C., 2018, High-water marks from Hurricane Sandy for coastal areas of Connecticut, Rhode Island, and Massachusetts, October 2012: U.S. Geological Survey Data Series 1094, 16 p., accessed May 2, 2019, at https://doi.org/10.3133/ ds 1094.

Rydlund, P.H., Jr., and Densmore, B.K., 2012, Methods of practice and guidelines for using survey-grade global navigation satellite systems (GNSS) to establish vertical datum in the United States Geological Survey: U.S. Geological Survey Techniques and Methods, book 11, chap. D1, 102 p., accessed December 24, 2013, at https://doi.org/10.3133/ tm11D1.

Samenow, J., 2018, Historic 'bomb cyclone' unleashes blizzard conditions from coastal Virginia to New England; frigid air to follow: The Washington Post, January 4, accessed May 7, 2019, at https://www.washingtonpost.com/ news/capital-weather-gang/wp/2018/01/04/historic-bombcyclone-unleashes-blizzard-conditions-from-coastalvirginia-to-new-england-frigid-air-to-follow.

Sauer, V.B., and Turnipseed, D.P., 2010, Stage measurement at gaging stations: U.S. Geological Survey Techniques and Methods book 3, chap. A7, 45 p., accessed September 12, 2019, at https://doi.org/10.3133/tm3A7.

U.S. Army Corps of Engineers, 2019, NAEregulated river basins: U.S. Army Corps of Engineers data, accessed September 23, 2019, at https://reservoircontrol.usace.army.mil/NE/pls/cwmsweb/ cwms_web.cwmsweb.cwmsindex.

U.S. Geological Survey, 2019a, Crest gage-A quick way to measure river stage: U.S. Geological Survey website, accessed September 12, 2019, at https://www.usgs.gov/ special-topic/water-science-school/science/crest-gage-aquick-way-measure-river-stage?qt-science_center_objects= 0\#qt-science_center_objects.
U.S. Geological Survey, 2019b, Nor'easter January 2018: U.S. Geological Survey Flood Event Viewer website, accessed September 12, 2019, at https://stn.wim.usgs.gov/ FEV/\#NoreasterJanuary2018.

U.S. Geological Survey, 2019c, Nor'easter of March 2018: U.S. Geological Survey Flood Event Viewer website, accessed September 12, 2019, at https://stn.wim.usgs.gov/ fev/\#NoreasterofMarch2018.

U.S. Geological Survey, 2019d, Water data for the nation: U.S. Geological Survey National Water Information System database, accessed September 19, 2019, at https://doi.org/ 10.5066/F7P55KJN.

Verdi, R.J., Lotspeich, R.R., Robbins, J.C., Busciolano, R.J., Mullaney, J.R., Massey, A.J., Banks, W.S., Roland, M.A., Jenter, H.L., Peppler, M.C., Suro, T.P., Schubert, C.E., and Nardi, M.R., 2017, The surge, wave, and tide hydrodynamics (SWaTH) network of the U.S. Geological Survey-Past and future implementation of storm-response monitoring, data collection, and data delivery: U.S. Geological Survey Circular 1431, 35 p., accessed September 12, 2019, at https://doi.org/10.3133/cir1431.

Wade, C.M., 2018, Baker seeks federal disaster funds for storm damages: The Eagle Tribune web page, May 1, accessed April 25, 2019, at https://www.eagletribune.com/ news/merrimack_valley/baker-seeks-federal-disaster-fundsfor-storm-damages/article_d2f0c7b4-bd75-5a8b-8a0c4dedbe44a7b4.html.

Weather Channel, The, 2018, Winter storm Riley underwent bombogenesis, battered the east with strong winds, coastal flooding, and heavy, wet snow (recap): The Weather Channel, March 4, accessed April 25, 2019, at https://weather.com/storms/winter/news/2018-03-01winter-storm-riley-noreaster-high-winds-coastal-floodingheavy-snow. 



\section{Appendix 1. Quality Assurance of Survey Equipment Used To Determine Elevations of High-Water Marks From the January and March 2018 Nor'easters}


Table 1.1. Quality assurance of survey equipment used by the U.S. Geological Survey to determine elevations of high-water marks from the January and March 2018 nor'easters relative to National Geodetic Survey and Massachusetts Department of Transportation certified benchmarks in Massachusetts and Rhode Island from August through

November 2018 and February 2019

[Blank spaces indicate information identical to the completed row above. NGS, National Geodetic Survey; MassDOT, Massachusetts Department of Transportation; USGS, U.S. Geological Survey; USCG, U.S. Coast Guard; PID, permanent identification; Vert., vertical can be first [1st] or second [2d]; NAD 83, North American Datum of 1983; NAVD 88, North American Vertical Datum of 1988; ft, foot; MA, Massachusetts; NH, New Hampshire; RI, Rhode Island; - , no data]

\begin{tabular}{|c|c|c|c|c|c|c|c|c|c|c|c|c|c|}
\hline \multirow{2}{*}{ Date surveyed } & \multicolumn{7}{|c|}{ NGS and MassDOT benchmark¹ } & \multicolumn{2}{|c|}{ Surveyed, in NAD 83} & \multirow{2}{*}{$\begin{array}{l}\text { Surveyed } \\
\text { eleva- } \\
\text { tion, in } \\
\mathrm{ft} \text { above } \\
\text { NAVD } 88\end{array}$} & \multirow{2}{*}{$\begin{array}{l}\text { NGS and } \\
\text { MassDOT } \\
\text { benchmark } \\
\text { elevation, } \\
\text { in ft above } \\
\text { NAVD } 88\end{array}$} & \multicolumn{2}{|c|}{$\begin{array}{c}\text { Difference between } \\
\text { NGS and MassDOT } \\
\text { and surveyed eleva- } \\
\text { tion, in ft }\end{array}$} \\
\hline & Owner & $\begin{array}{l}\text { Designation } \\
\text { (stamped } \\
\text { name) }\end{array}$ & PID & $\begin{array}{l}\text { Vert. } \\
\text { order }^{2}\end{array}$ & County & Town & State & $\begin{array}{l}\text { Latitude, } \\
\text { in decimal } \\
\text { degrees }\end{array}$ & $\begin{array}{c}\text { Longitude, } \\
\text { in decimal } \\
\text { degrees }\end{array}$ & & & Relative & Absolute \\
\hline \multirow[t]{3}{*}{$8 / 28 / 2018$} & NGS & $22 \mathrm{~K}$ & MY0150 & $1 \mathrm{st}$ & Essex & Newburyport & MA & 42.80223 & 70.87950 & 31.37 & 31.36 & -0.01 & 0.01 \\
\hline & & & & & & & & & & 31.32 & 31.36 & 0.04 & 0.04 \\
\hline & & & & & & & & & & 31.41 & 31.36 & -0.05 & 0.05 \\
\hline \multirow[t]{2}{*}{$8 / 29 / 2018$} & MassDOT & - & 24885 & $2 \mathrm{~d}$ & Essex & Essex & MA & 42.62874 & 70.77094 & 19.00 & 18.95 & -0.05 & 0.05 \\
\hline & & & & & & & & & & 18.71 & 18.95 & 0.24 & 0.24 \\
\hline \multirow[t]{2}{*}{$8 / 30 / 2018$} & MassDOT & - & 24885 & $2 d$ & Essex & Essex & MA & 42.62874 & 70.77094 & 18.95 & 18.95 & 0.00 & 0.00 \\
\hline & & & & & & & & & & 19.07 & 18.95 & -0.12 & 0.12 \\
\hline \multirow[t]{2}{*}{$8 / 31 / 2018$} & MassDOT & - & 25282 & $2 d$ & Essex & Salem & MA & 42.50832 & 70.91286 & 93.24 & 93.28 & 0.04 & 0.04 \\
\hline & & & & & & & & & & 93.24 & 93.28 & 0.04 & 0.04 \\
\hline \multirow[t]{2}{*}{$9 / 4 / 2018$} & NGS & Langdon & $\mathrm{OC} 0425$ & $2 d$ & Rockingham & Portsmouth & $\mathrm{NH}$ & 43.06345 & 70.71189 & 10.01 & 9.87 & -0.14 & 0.14 \\
\hline & & $\begin{array}{l}146 \mathrm{RM} \\
1\end{array}$ & & & & & & & & 9.74 & 9.87 & 0.13 & 0.13 \\
\hline \multirow[t]{2}{*}{$9 / 5 / 2018$} & NGS & 8 & MY0586 & $1 \mathrm{st}$ & Suffolk & Boston & MA & 42.37438 & 71.05468 & 8.76 & 8.87 & 0.11 & 0.11 \\
\hline & & & & & & & & & & 8.93 & 8.87 & -0.06 & 0.06 \\
\hline \multirow[t]{2}{*}{$9 / 6 / 2018$} & NGS & 8 & MY0586 & $1 \mathrm{st}$ & Suffolk & Boston & MA & 42.37438 & 71.05468 & 8.97 & 8.87 & -0.10 & 0.10 \\
\hline & & & & & & & & & & 8.79 & 8.87 & 0.08 & 0.08 \\
\hline \multirow[t]{2}{*}{ 9/17/2018 } & NGS & 8 & MY0586 & $1 \mathrm{st}$ & Suffolk & Boston & MA & 42.37438 & 71.05468 & 8.77 & 8.87 & 0.10 & 0.10 \\
\hline & & & & & & & & & & 8.74 & 8.87 & 0.13 & 0.13 \\
\hline \multirow[t]{2}{*}{$9 / 18 / 2018$} & NGS & $85 \mathrm{AR}$ & MY2172 & $1 \mathrm{st}$ & Norfolk & Weymouth & MA & 42.24252 & 70.96043 & 29.43 & 29.42 & -0.01 & 0.01 \\
\hline & & & & & & & & & & 29.52 & 29.42 & -0.09 & 0.09 \\
\hline \multirow[t]{2}{*}{ 9/19/2018 } & NGS & $85 \mathrm{AR}$ & MY2172 & $1 \mathrm{st}$ & Norfolk & Weymouth & MA & 42.24252 & 70.96043 & 29.35 & 29.42 & 0.07 & 0.07 \\
\hline & & & & & & & & & & 29.38 & 29.42 & 0.04 & 0.04 \\
\hline
\end{tabular}


Table 1.1. Quality assurance of survey equipment used by the U.S. Geological Survey to determine elevations of high-water marks from the January and March 2018 nor'easters relative to National Geodetic Survey and Massachusetts Department of Transportation certified benchmarks in Massachusetts and Rhode Island from August through

November 2018 and February 2019.-Continued

[Blank spaces indicate information identical to the completed row above. NGS, National Geodetic Survey; MassDOT, Massachusetts Department of Transportation; USGS, U.S. Geological Survey; USCG, U.S Coast Guard; PID, permanent identification; Vert., vertical can be first [1 st] or second [2d]; NAD 83, North American Datum of 1983; NAVD 88, North American Vertical Datum of 1988; ft, foot; MA, Massachusetts; NH, New Hampshire; RI, Rhode Island; - , no data]

\begin{tabular}{|c|c|c|c|c|c|c|c|c|c|c|c|c|c|}
\hline \multirow[t]{2}{*}{ Date surveyed } & \multicolumn{7}{|c|}{ NGS and MassDOT benchmark1 } & \multicolumn{2}{|c|}{ Surveyed, in NAD 83} & \multirow{2}{*}{$\begin{array}{c}\text { Surveyed } \\
\text { eleva- } \\
\text { tion, in } \\
\mathrm{ft} \text { above } \\
\text { NAVD } 88\end{array}$} & \multirow{2}{*}{$\begin{array}{c}\text { NGS and } \\
\text { MassDOT } \\
\text { benchmark } \\
\text { elevation, } \\
\text { in ft above } \\
\text { NAVD } 88\end{array}$} & \multicolumn{2}{|c|}{$\begin{array}{c}\text { Difference between } \\
\text { NGS and MassDOT } \\
\text { and surveyed eleva- } \\
\text { tion, in ft }\end{array}$} \\
\hline & Owner & $\begin{array}{c}\text { Designation } \\
\text { (stamped } \\
\text { name) }\end{array}$ & PID & $\begin{array}{c}\text { Vert. } \\
\text { order }^{2}\end{array}$ & County & Town & State & $\begin{array}{l}\text { Latitude, } \\
\text { in decimal } \\
\text { degrees }\end{array}$ & $\begin{array}{c}\text { Longitude, } \\
\text { in decimal } \\
\text { degrees }\end{array}$ & & & Relative & Absolute \\
\hline \multirow[t]{3}{*}{ 9/20/2018 } & NGS & $85 \mathrm{AR}$ & MY2172 & $1 \mathrm{st}$ & Norfolk & Weymouth & MA & 42.24252 & 70.96043 & 29.34 & 29.42 & 0.08 & 0.08 \\
\hline & & & & & & & & & & 29.28 & 29.42 & 0.14 & 0.14 \\
\hline & & & & & & & & & & 29.28 & 29.42 & 0.14 & 0.14 \\
\hline \multirow[t]{3}{*}{$9 / 25 / 2018$} & NGS & 342 & MY2164 & $1 \mathrm{st}$ & Plymouth & Hingham & MA & 42.24634 & 70.88189 & 9.77 & 9.82 & 0.05 & 0.05 \\
\hline & & & & & & & & & & 9.90 & 9.82 & -0.08 & 0.08 \\
\hline & & & & & & & & & & 9.83 & 9.82 & -0.01 & 0.01 \\
\hline \multirow[t]{3}{*}{$9 / 27 / 2018$} & NGS & $\mathrm{N} 42$ & MY2151 & $1 \mathrm{st}$ & Plymouth & Marshfield & MA & 42.14719 & 70.74291 & 115.39 & 115.34 & -0.05 & 0.05 \\
\hline & & & & & & & & & & 115.36 & 115.34 & -0.02 & 0.02 \\
\hline & & & & & & & & & & 115.37 & 115.34 & -0.02 & 0.02 \\
\hline \multirow[t]{2}{*}{$10 / 10 / 2018$} & NGS & Q 43 & LW1524 & $1 \mathrm{st}$ & Plymouth & Manomet & MA & 41.80298 & 70.54562 & 122.62 & 122.83 & 0.21 & 0.21 \\
\hline & & & & & & & & & & 122.62 & 122.83 & 0.21 & 0.21 \\
\hline \multirow[t]{2}{*}{$10 / 10 / 2018$} & NGS & D 56 & AB7923 & $1 \mathrm{st}$ & Barnstable & Marstons & MA & 41.65106 & 70.41449 & 24.61 & 24.53 & -0.08 & 0.08 \\
\hline & & & & & & Mills & & & & 24.54 & 24.53 & -0.01 & 0.01 \\
\hline \multirow[t]{2}{*}{$10 / 11 / 2018$} & MassDOT & - & 17534 & $2 d$ & Barnstable & Provincetown & MA & 42.05908 & 70.18903 & 15.40 & 15.47 & 0.07 & 0.07 \\
\hline & & & & & & & & & & 15.32 & 15.47 & 0.15 & 0.15 \\
\hline \multirow[t]{2}{*}{$10 / 11 / 2018$} & USGS & - & HER- & - & Barnstable & Wellfleet & MA & 41.93115 & 70.06430 & 11.46 & 11.54 & 0.08 & 0.08 \\
\hline & & & $\begin{array}{l}\text { RING- } \\
\text { DIK- } \\
\text { ERM2 }\end{array}$ & & & & & & & 11.57 & 11.54 & -0.02 & 0.02 \\
\hline \multirow[t]{2}{*}{$10 / 11 / 2018$} & NGS & Chatham & AB2629 & $1 \mathrm{st}$ & Barnstable & Chatham & MA & 41.67174 & 69.94916 & 40.12 & 40.30 & 0.18 & 0.18 \\
\hline & & $\begin{array}{l}\text { Light } \\
\text { USCG }\end{array}$ & & & & & & & & 40.22 & 40.30 & 0.08 & 0.08 \\
\hline \multirow[t]{2}{*}{$10 / 30 / 2018$} & MassDOT & - & 10943 & $2 d$ & Plymouth & Marshfield & MA & 42.09301 & 70.69371 & 16.62 & 16.56 & -0.06 & 0.06 \\
\hline & & & & & & & & & & 16.56 & 16.56 & 0.00 & 0.00 \\
\hline
\end{tabular}


Table 1.1. Quality assurance of survey equipment used by the U.S. Geological Survey to determine elevations of high-water marks from the January and March 2018 nor'easters relative to National Geodetic Survey and Massachusetts Department of Transportation certified benchmarks in Massachusetts and Rhode Island from August through

November 2018 and February 2019._-Continued

[Blank spaces indicate information identical to the completed row above. NGS, National Geodetic Survey; MassDOT, Massachusetts Department of Transportation; USGS, U.S. Geological Survey; USCG, U.S. Coast Guard; PID, permanent identification; Vert., vertical can be first [1st] or second [2d]; NAD 83, North American Datum of 1983; NAVD 88, North American Vertical Datum of 1988; ft, foot; MA, Massachusetts; NH, New Hampshire; RI, Rhode Island; - , no data]

\begin{tabular}{|c|c|c|c|c|c|c|c|c|c|c|c|c|c|}
\hline \multirow[t]{2}{*}{ Date surveyed } & \multicolumn{7}{|c|}{ NGS and MassDOT benchmark1 } & \multicolumn{2}{|c|}{ Surveyed, in NAD 83} & \multirow{2}{*}{$\begin{array}{c}\text { Surveyed } \\
\text { eleva- } \\
\text { tion, in } \\
\mathrm{ft} \text { above } \\
\text { NAVD } 88\end{array}$} & \multirow{2}{*}{$\begin{array}{l}\text { NGS and } \\
\text { MassDOT } \\
\text { benchmark } \\
\text { elevation, } \\
\text { in ft above } \\
\text { NAVD } 88\end{array}$} & \multicolumn{2}{|c|}{$\begin{array}{c}\text { Difference between } \\
\text { NGS and MassDOT } \\
\text { and surveyed eleva- } \\
\text { tion, in ft }\end{array}$} \\
\hline & Owner & $\begin{array}{l}\text { Designation } \\
\text { (stamped } \\
\text { name) }\end{array}$ & PID & $\begin{array}{l}\text { Vert. } \\
\text { order }^{2}\end{array}$ & County & Town & State & $\begin{array}{l}\text { Latitude, } \\
\text { in decimal } \\
\text { degrees }\end{array}$ & $\begin{array}{l}\text { Longitude, } \\
\text { in decimal } \\
\text { degrees }\end{array}$ & & & Relative & Absolute \\
\hline \multirow[t]{2}{*}{$10 / 30 / 2018$} & NGS & G 2 & MY0196 & $1 \mathrm{st}$ & Rockingham & Seabrook & $\mathrm{NH}$ & 42.91180 & 70.85175 & 7.53 & 7.61 & 0.08 & 0.08 \\
\hline & & & & & & & & & & 7.61 & 7.61 & 0.00 & 0.00 \\
\hline \multirow[t]{2}{*}{$10 / 31 / 2018$} & MassDOT & - & 10875 & $2 d$ & Plymouth & Hanover & MA & 42.10439 & 70.79254 & 7.60 & 7.58 & -0.02 & 0.02 \\
\hline & & & & & & & & & & 7.42 & 7.58 & 0.15 & 0.15 \\
\hline \multirow[t]{2}{*}{$10 / 31 / 2018$} & MassDOT & - & 10943 & $2 d$ & Plymouth & Marshfield & MA & 42.09301 & 70.69371 & 16.49 & 16.56 & 0.07 & 0.07 \\
\hline & & & & & & & & & & 16.55 & 16.56 & 0.01 & 0.01 \\
\hline \multirow[t]{2}{*}{$11 / 1 / 2018$} & MassDOT & - & 4981 & $2 d$ & Barnstable & Orleans & MA & 41.78833 & 69.99147 & 58.69 & 58.64 & -0.04 & 0.04 \\
\hline & & & & & & & & & & 58.68 & 58.64 & -0.03 & 0.03 \\
\hline \multirow[t]{2}{*}{$11 / 1 / 2018$} & NGS & 8446493 & LW1544 & $1 \mathrm{st}$ & Plymouth & Plymouth & MA & 41.95948 & 70.66243 & 9.99 & 9.98 & -0.01 & 0.01 \\
\hline & & Tidal 10 & & & & & & & & 9.97 & 9.98 & 0.01 & 0.01 \\
\hline \multirow[t]{2}{*}{$11 / 02 / 208$} & MassDOT & - & 17409 & $2 d$ & Barnstable & Dennis & MA & 41.73747 & 70.19138 & 37.90 & 38.00 & 0.10 & 0.10 \\
\hline & & & & & & & & & & 37.92 & 38.00 & 0.07 & 0.07 \\
\hline \multirow[t]{2}{*}{ 2/8/2019 } & NGS & 440 & LW0561 & $2 d$ & Bristol & Bristol & RI & 41.70240 & 71.28000 & 114.50 & 114.44 & -0.06 & 0.06 \\
\hline & & & & & & & & & & 114.41 & 114.44 & 0.03 & 0.03 \\
\hline Minimum & & & & & & & & & & & & -0.14 & \\
\hline Maximum & & & & & & & & & & & & 0.24 & \\
\hline Median & & & & & & & & & & & & & 0.06 \\
\hline Average & & & & & & & & & & & & & 0.07 \\
\hline
\end{tabular}

1Massachusetts Department of Transportation (2018) and National Oceanic and Atmospheric Administration (2018c).

${ }^{2}$ NGS standards assign order to express the accuracy of established vertical control to local reference point in the level circuit (National Oceanic and Atmospheric Administration, 2020). 


\section{References Cited}

Massachusetts Department of Transportation, 2018, Geodetic control: Massachusetts Department of Transportation map viewer, accessed August 21, 2018, at https://gis.massdot.state.ma.us/geodeticcontrol/.

National Oceanic and Atmospheric Administration, 2018c, Where is the highest tide?: National Oceanic and Atmospheric Administration web page, August 13, accessed April 22, 2019, at https://oceanservice.noaa.gov/facts/ highesttide.html.

National Oceanic and Atmospheric Administration, 2020, The DSDATA format: National Oceanic and Atmospheric Administration data sheet, 46 p., accessed February 21, 2020, at https://www.ngs.noaa.gov/DATASHEET/

dsdata.pdf. 

For more information about this report, contact: Director, New England Water Science Center U.S. Geological Survey

10 Bearfoot Road

Northborough, MA 01532

dc_nweng@usgs.gov

or visit our website at

https://www.usgs.gov/centers/new-england-water

Publishing support provided by the

Pembroke Publishing Service Center 
